\title{
Semi-local string comparison: Algorithmic techniques and applications
}

\author{
Alexander Tiskin ${ }^{1}$
}

May 15, 2022

${ }^{1}$ Department of Computer Science, University of Warwick, Coventry CV4 7AL, United Kingdom. Research supported by the Centre for Discrete Mathematics and Its Applications (DIMAP), University of Warwick. 


\begin{abstract}
The longest common subsequence (LCS) problem is a classical problem in computer science. The semi-local LCS problem is a generalisation of the LCS problem, arising naturally in the context of string comparison. In this work, we present a number of algorithmic techniques related to the semi-local LCS problem, and give a number of algorithmic applications of these techniques. Summarising the presented results, we conclude that semilocal string comparison turns out to be a useful algorithmic plug-in, which unifies, and often improves on, a number of previous approaches to various substring- and subsequence-related problems.
\end{abstract}




\section{Contents}

1 Introduction $\quad 3$

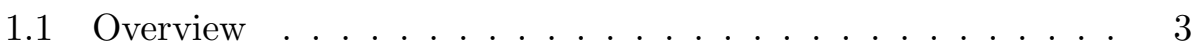

1.2 Points and matrices _............... 5

1.3 Distribution and density matrices . . . . . . . . . 8

1.4 Permutation and unit-Monge matrices . . . . . . . . . 9

2 Matrix distance multiplication $\quad 12$

2.1 Distance multiplication monoids . . . . . . . . . . . 12

2.2 Seaweed braids . . . . . . . . . . . . . . 15

2.3 Distance multiplication algorithms . . . . . . . . . 17

2.4 Technical lemmas . . . . . . . . . . . . . . . . 23

3 Semi-local string comparison $\quad 28$

3.1 Semi-local LCS . . . . . . . . . . . . . . . . . . 28

3.2 Alignment dags and highest-score matrices . . . . . . . . . . 30

3.3 Score matrix composition . . . . . . . . . . . . 36

3.4 Weighted scores and edit distances . . . . . . . . . . . . 40

4 The seaweed method 44

4.1 The seaweed algorithm . . . . . . . . . . . . . . . 44

4.2 Incremental LCS and semi-local LCS . . . . . . . . . . . 47

4.3 Common-substring LCS and semi-local LCS . . . . . . . . . 48

5 The micro-block seaweed method $\quad 50$

5.1 The micro-block seaweed algorithm . . . . . . . . . 50

5.2 Cyclic LCS . . . . . . . . . . . . . . . . . 53

5.3 Longest repeating subsequence . . . . . . . . . . . . 54

5.4 Approximate pattern matching . . . . . . . . . . . 54

6 Periodic string comparison $\quad 57$

6.1 The periodic seaweed algorithm . . . . . . . . . 57

6.2 Tandem alignment . . . . . . . . . . . . . . . . 59 
7 Permutation string comparison $\quad 62$

7.1 Semi-local LCS between permutations . . . . . . . . . . 62

7.2 Cyclic LCS between permutations . . . . . . . . . . 64

7.3 Longest pattern-avoiding subsequences . . . . . . . . . . 65

7.4 Longest piecewise monotone subsequences . . . . . . . . 66

7.5 Maximum clique in a circle graph . . . . . . . . . . 67

7.6 Maximum common pattern between linear graphs . . . . . . 71

8 Compressed string comparison $\quad \mathbf{7 3}$

8.1 Grammar-compressed strings . . . . . . . . . . . . 73

8.2 Global subsequence recognition . . . . . . . . . . . . 74

8.3 Three-way semi-local LCS . . . . . . . . . . . . . . 75

8.4 Local subsequence recognition . . . . . . . . . . . . 77

9 Beyond semi-locality $\quad 81$

9.1 Window-local LCS and alignment plots . . . . . . . . 81

9.2 Quasi-local LCS . . . . . . . . . . . . . . . . . . . . 85

9.3 Sparse spliced alignment . . . . . . . . . . . 87

$\begin{array}{ll}10 \text { Conclusions } & 90\end{array}$

11 Acknowledgement $\quad 91$ 


\section{Chapter 1}

\section{Introduction}

\subsection{Overview}

The longest common subsequence (LCS) problem is a classical problem in computer science. Given two strings $a, b$ of lengths $m, n$ respectively, the LCS problem asks for the length of the longest string that is a subsequence of both $a$ and $b$. This length is called the strings' LCS score. We refer the reader to monographs $[35,54]$ for the background and further references.

The semi-local LCS problem is a generalisation of the LCS problem, arising naturally in the context of string comparison. Given two strings $a, b$ as before, the semi-local LCS problem asks for the LCS score of each string against all substrings of the other string, and of all prefixes of each string against all suffixes of the other string. In this work, we survey a number of algorithmic techniques related to the semi-local LCS problem, and present some algorithmic applications of these techniques.

The rest of this chapter contains the necessary preliminaries. In Section 1.2, we establish the basic terminology and notation of points and matrices. In Section 1.3, we give the key definitions of distribution and density matrices. In Section 1.4, we introduce our main algorithmic tool: a special class of integer matrices, called simple unit-Monge matrices, which are obtained as distribution matrices of permutation matrices, and can be represented implicitly by a dominance counting data structure, such as a range tree.

In Chapter 2, we describe our main algorithmic techniques. In Section 2.1, we introduce matrix distance multiplication, and study its algebraic properties in the classes of Monge and simple unit-Monge matrices. In Section 2.2, we present an algebraic formalism for distance multiplication of simple unit-Monge matrices, called seaweed braids. In Section 2.3, we give an efficient algorithm for distance multiplication of implicit simple unit-Monge matrices (or, equivalently, seaweed braids). In Section 2.4, we prove two technical lemmas required for subsequent applications. 
In Chapter 3, we develop an application of our techniques to the semilocal LCS problem. In Section 3.1, we formally define the semi-local LCS problem and related concepts. In Section 3.2, we introduce alignment dags and highest-score matrices. Exploiting the algebraic framework of unitMonge matrices, in Section 3.3 we obtain an efficient algorithm for highestscore matrix composition. In Section 3.4, we generalise our techniques from LCS scores to arbitrary rational-weighted alignment scores and edit distances.

In Chapter 4, we introduce a conceptually simple algorithm for the semilocal LCS problem, called the seaweed algorithm, and show a number of its applications. In Section 4.1, we describe the seaweed algorithm itself, and in Sections 4.2 and 4.3, we apply it to solving the incremental and the commonsubstring versions of the LCS and semi-local LCS problems. Our algorithms match, improve on, or generalise existing algorithms for these problems.

In Chapter 5, we give a micro-block version of the seaweed algorithm, which is slightly faster than the plain seaweed algorithm, and gives rise to further applications. In Section 5.1, we describe the micro-block seaweed algorithm itself. In the case of an unbounded-size alphabet, the running time of the micro-block seaweed algorithm matches the running time of the best known global LCS algorithm, while providing a much more detailed string comparison. By direct application of the micro-block seaweed algorithm, in Section 5.2 we obtain an improved algorithm for the cyclic LCS problem, and in Section 5.3 for the longest repeating subsequence problem. In Section 5.4, we consider the approximate pattern matching problem, and apply the micro-block seaweed algorithm to obtain an algorithm for this problem, matching the best known algorithm in running time.

In Chapter 6, we describe an extension of the seaweed algorithm that allows efficient semi-local comparison of two input strings, one of which is periodic. In Section 6.1, we describe the periodic seaweed algorithm itself. By application of the periodic seaweed algorithm, in Section 6.2 we obtain new algorithms for the tandem LCS problem and the tandem cyclic alignment problem, improving on existing algorithms in running time.

In Chapter 7, we consider the semi-local LCS problem restricted to permutation strings of length $n$. In particular, Section 7.1 gives an algorithm for the semi-local LCS problem on permutation strings. By direct application of this algorithm, in Section 7.2 we obtain an improved algorithm for the cyclic LCS problem on permutations. Further applications include improved algorithms for the longest pattern-avoiding subsequence problem, given in Section 7.3, and for the longest $k$-increasing and $k$-modal subsequence problems, given in Section 7.4. In Section 7.5, we consider the maximum clique problem in a circle graph represented by an interval model of size $n$. By application our semi-local LCS algorithm on permutations, we obtain new algorithms for this problem, both for general and sparse circle graphs, achieving a substantial improvement on existing algorithms in run- 
ning time. In Section 7.6, we describe an application of these algorithms to the problem of finding exact and approximate commonly structured patterns in linear graphs.

In Chapter 8, we apply the semi-local LCS problem to compressed string comparison. Our goal is to obtain efficient algorithms that work on compressed strings without first decompressing them. In Section 8.1, we introduce the grammar compression (GC) framework, that generalises the classical LZ78 and LZW methods. In Section 8.2, we describe a folklore algorithm for global subsequence recognition on GC-strings. In Section 8.3, we give an efficient algorithm for the three-way semi-local LCS problem on GC-strings. By application of this algorithm, in Section 8.4 we obtain an algorithm for local subsequence recognition in GC-strings, which improves on existing algorithms in running time.

In Chapter 9, we consider applications of our techniques that aim to reach beyond semi-local string comparison, with the ultimate goal of efficient fully-local comparison. In Section 9.1, we introduce the windowsubstring and window-window LCS problems, and give an algorithm for these problems. This algorithm provides a refinement for the standard dot plot method, by allowing efficient window-window string comparison based on the LCS score, rather than the less sensitive Hamming score. In Section 9.2, we introduce the quasi-local LCS problem, which generalises the semi-local, window-substring and window-window LCS problems, and give an efficient algorithm for this problem. By application of this algorithm, in Section 9.3 we obtain an algorithm for sparse spliced alignment under an arbitrary rational edit distance metric, which improves on existing algorithms for this problem.

Some results presented in this work appeared incrementally in the author's publications $[105,106,108,107,109,72,110]$. The aim of this work is to consolidate these results, unifying the terminology and notation. However, a number of results have not been published before, and are original to this work.

Summarising the presented results, we conclude that semi-local string comparison turns out to be a useful algorithmic plug-in, which unifies, and often improves on, a number of previous approaches to various substringand subsequence-related problems.

\subsection{Points and matrices}

In addition to integers $\{\ldots,-2,-1,0,1,2, \ldots\}$, we will use odd half-integers $\left\{\ldots,-\frac{5}{2},-\frac{3}{2},-\frac{1}{2}, \frac{1}{2}, \frac{3}{2}, \frac{5}{2}, \ldots\right\}$. For ease of reading, odd half-integer variables will be indicated by hats (e.g. $\hat{\imath}, \hat{\jmath})$. Ordinary variable names (e.g. $i, j$, with possible subscripts or superscripts), will normally indicate integer variables, but can sometimes indicate a variable that may be either integer, or odd 
half-integer.

We denote integer and odd half-integer intervals by

$$
\begin{aligned}
& {[i: j]=\{i, i+1, \ldots, j-1, j\}} \\
& \langle i: j\rangle=\left\{i+\frac{1}{2}, i+\frac{3}{2}, \ldots, j-\frac{3}{2}, j-\frac{1}{2}\right\}
\end{aligned}
$$

Note that in this notation, both an integer and an odd half-integer interval is defined by integer endpoints. To denote infinite intervals of integers and odd half-integers, we will use $-\infty$ for $i$ and $+\infty$ for $j$ where appropriate, so e.g. $[-\infty:+\infty]$ denotes the set of all integers, and $\langle-\infty:+\infty\rangle$ the set of all odd half-integers. For finite intervals $[i: j]$ and $\langle i: j\rangle$, we call the difference $j-i$ interval length.

When dealing with pairs of numbers, we will often use geometric language and call them points. We will write

$$
\begin{array}{ll}
\left(i_{0}, j_{0}\right) \ll\left(i_{1}, j_{1}\right) & \text { if } i_{0}<i_{1} \text { and } j_{0}<j_{1} \\
\left(i_{0}, j_{0}\right) \lessgtr\left(i_{1}, j_{1}\right) & \text { if } i_{0}<i_{1} \text { and } j_{0}>j_{1}
\end{array}
$$

We will call these strict partial orders $\ll$ - and $\lessgtr$-dominance. When visualising points, we will use the matrix indexing convention: the first coordinate in a pair increases downwards, and the second coordinate rightwards. Hence, the standard visual convention of the dominated point lying "below and to the left" of the dominating point corresponds in this work to $\lessgtr$-dominance.

We use standard terminology for geometric dominance and other partial orders. In particular, a set of elements forms a chain, if they are pairwise comparable, and an antichain, if they pairwise incomparable. Note that a «-chain is a $\lessgtr$-antichain, and vice versa. An element in a partially ordered set is minimal, if does not dominate any other element in the set (in terms of the partial order). All minimal elements in a partially ordered set form an antichain.

A function of an integer argument will be called unit-monotone increasing (respectively, decreasing), if the difference between every pair of successive values is either 0 or 1 (respectively, 0 or -1 ).

We will make extensive use of vectors and matrices with integer (occasionally, rational or real) elements, and with integer or odd half-integer indices. We regard a vector or matrix as a one- (respectively, two-) argument function, so we can speak e.g. about unit-monotone increasing matrices.

A vector or matrix is nonnegative, if all its elements are nonnegative. We will often consider matrices where one or both index range are nonconsecutive sets of integers or odd half-integers; however, index ranges will always be assumed to be linearly ordered. Given two index ranges $I, J$, it will be convenient to denote their Cartesian product by $(I \mid J)$. We extend this notation to Cartesian products of intervals:

$$
\left[i_{0}: i_{1} \mid j_{0}: j_{1}\right]=\left(\left[i_{0}: i_{1}\right] \mid\left[j_{0}: j_{1}\right]\right)
$$




$$
\left\langle i_{0}: i_{1} \mid j_{0}: j_{1}\right\rangle=\left(\left\langle i_{0}: i_{1}\right\rangle \mid\left\langle j_{0}: j_{1}\right\rangle\right)
$$

Given finite or infinite index ranges $I, J$, a vector over $I$ is indexed by $i \in I$, and a matrix over $(I \mid J)$ is indexed by $i \in I, j \in J$.

The matrices we consider can be implicit, i.e. represented by a compact data structure that supports access to every matrix element in a specified (not necessarily constant) time. If the query time is not given, it is assumed by default to be constant.

When considering matrices over non-consecutive index ranges, we will occasionally perform operations on such matrices as if they were over consecutive intervals. This will have the following meaning: we remap the ranges to consecutive intervals preserving the linear order within each range, then we perform a matrix operation, and finally we remap the intervals back to the original ranges.

We will use parenthesis notation for indexing matrices, e.g. $A(i, j)$. We will use straightforward notation for selecting subvectors and submatrices; for example, given a matrix $A$ over $[0: n]^{2}$, we denote by $A\left[i_{0}: i_{1} \mid j_{0}: j_{1}\right]$ the submatrix defined by the given sub-intervals. A star $*$ will indicate that for a particular index, its whole range is selected implicitly, e.g. $A\left[* \mid j_{0}\right.$ : $\left.j_{1}\right]=A\left[0: n \mid j_{0}: j_{1}\right]$.

Given matrices $A^{\prime}$ over $\left(I^{\prime} \mid J^{\prime}\right)$ and $A^{\prime \prime}$ over $\left(I^{\prime \prime} \mid J^{\prime \prime}\right)$, where $\left(I^{\prime} \mid J^{\prime}\right) \cap$ $\left(I^{\prime \prime} \mid J^{\prime \prime}\right)=\emptyset$, their disjoint union is the matrix $A^{\prime} \sqcup A^{\prime \prime}$ over $\left(I^{\prime} \cup I^{\prime \prime} \mid J^{\prime} \cup J^{\prime \prime}\right)$, defined by

$$
\left(A^{\prime} \sqcup A^{\prime \prime}\right)(i, j)= \begin{cases}A^{\prime}(i, j) & \text { if } i \in I^{\prime}, j \in J^{\prime} \\ A^{\prime \prime}(i, j) & \text { if } i \in I^{\prime \prime}, j \in J^{\prime \prime} \\ 0 & \text { otherwise }\end{cases}
$$

The matrix disjoint union operation is associative, and can therefore be used for more than two matrices.

Given matrices $A$ over $(I \mid J)$ and $A^{\prime}$ over $\left(I^{\prime} \mid J^{\prime}\right)$, where $I \supset I^{\prime}$ and $J \supset J^{\prime}$, the corresponding matrix embedding is the matrix $A \sqsupset A^{\prime}$ over $(I \mid J)$, defined by

$$
\left(A \sqsupset A^{\prime}\right)(i, j)= \begin{cases}A^{\prime}(i, j) & \text { if } i \in I^{\prime}, j \in J^{\prime} \\ A(i, j) & \text { otherwise }\end{cases}
$$

Matrix $A^{\prime}$, when regarded as a submatrix of $A \sqsupset A^{\prime}$, will be called the core of the embedding. 


\subsection{Distribution and density matrices}

Definition 1 Let $D$ be a matrix over $\left\langle i_{0}: i_{1} \mid j_{0}: j_{1}\right\rangle$. Its distribution matrix $D^{\Sigma}$ over $\left[i_{0}: i_{1} \mid j_{0}: j_{1}\right]$ is defined by

$$
D^{\Sigma}(i, j)=\sum_{\hat{\imath} \in\left\langle i: i_{1}\right\rangle, \hat{\jmath} \in\left\langle j_{0}: j\right\rangle} D(\hat{\imath}, \hat{\jmath})
$$

for all $i \in\left[i_{0}: i_{1}\right], j \in\left[j_{0}: j_{1}\right]$.

Definition 2 Let $A$ be a matrix over $\left[i_{0}: i_{1} \mid j_{0}: j_{1}\right]$. Its density matrix $A^{\square}$ over $\left\langle i_{0}: i_{1} \mid j_{0}: j_{1}\right\rangle$ is defined by

$$
\begin{aligned}
A^{\square}(\hat{\imath}, \hat{\jmath})= & A\left(\hat{\imath}+\frac{1}{2}, \hat{\jmath}-\frac{1}{2}\right)-A\left(\hat{\imath}-\frac{1}{2}, \hat{\jmath}-\frac{1}{2}\right)- \\
& A\left(\hat{\imath}+\frac{1}{2}, \hat{\jmath}+\frac{1}{2}\right)+A\left(\hat{\imath}-\frac{1}{2}, \hat{\jmath}+\frac{1}{2}\right)
\end{aligned}
$$

for all $\hat{\imath} \in\left\langle i_{0}: i_{1}\right\rangle, \hat{\jmath} \in\left\langle j_{0}: j_{1}\right\rangle$.

Note that for any matrix $D$ as above, and for all $\hat{\imath}, \hat{\jmath}$, we have

$$
\left(D^{\Sigma}\right)^{\square}(\hat{\imath}, \hat{\jmath})=D(\hat{\imath}, \hat{\jmath})
$$

Also, for any matrix $A$ as above, we have

$$
\left(A^{\square}\right)^{\Sigma}(i, j)+b(i)+c(j)=A(i, j)
$$

for all $i, j$, where $b(i)=A\left(i, j_{0}\right), c(j)=A\left(i_{1}, j\right)$. An important special case is when $b, c$ are both zero vectors, as in the following definition.

Definition 3 Matrix $A$ will be called simple, if $\left(A^{\square}\right)^{\Sigma}=A$.

Equivalently, a (finite) matrix is simple, if and only if its entries in the leftmost column and the bottom row are all zeros.

The following classes of matrices play a fundamental role in optimisation theory (see [22] for an extensive survey), as well as in graph and string algorithms.

Definition 4 Matrix $A$ is called totally monotone, if

$$
A(i, j)>A\left(i, j^{\prime}\right): A\left(i^{\prime}, j\right)>A\left(i^{\prime}, j^{\prime}\right)
$$

for all $i \leq i^{\prime}, j \leq j^{\prime}$.

Definition 5 Matrix $A$ is called a Monge matrix, if

$$
A(i, j)+A\left(i^{\prime}, j^{\prime}\right) \leq A\left(i, j^{\prime}\right)+A\left(i^{\prime}, j\right)
$$

for all $i \leq i^{\prime}, j \leq j^{\prime}$. Equivalently, matrix $A$ is a Monge matrix, if $A^{\square}$ is nonnegative. Matrix $A$ is called an anti-Monge matrix, if $-A$ is Monge.

It is easy to see that Monge matrices form a subclass of totally monotone matrices. By Definition 5, a matrix is Monge, if and only if its density matrix is nonnegative. This condition is equivalent to the canonical structure theorem for Monge matrices, given by Burkard et al. [22]. 


\subsection{Permutation and unit-Monge matrices}

A permutation (respectively, subpermutation) matrix is a zero-one matrix containing exactly one (respectively, at most one) nonzero in every row and every column. Typically, permutation and subpermutation matrices will be indexed by (not necessarily consecutive) odd half-integers. Given sets $I, J$ of odd half-integers with $|I|=|J|$, a zero-one matrix $P$ over $(I \mid J)$ is a permutation matrix, if and only if

$$
\sum_{\hat{\jmath}^{\prime} \in J} P\left(\hat{\imath}, \hat{\jmath}^{\prime}\right)=1 \quad \sum_{\hat{\imath}^{\prime} \in I} P\left(\hat{\imath}^{\prime}, \hat{\jmath}\right)=1
$$

for all $\hat{\imath} \in I, \hat{\jmath} \in J$. An identity matrix is a permutation matrix Id over an interval range $\left\langle i_{0}: i_{1} \mid i_{0}: i_{1}\right\rangle$, such that $\operatorname{Id}(\hat{\imath}, \hat{\jmath})=1$, iff $\hat{\imath}=\hat{\jmath}$. More generally, an offset indentity matrix is a permutation matrix $I d_{h}$ over an interval range $\left\langle i_{0}: i_{1} \mid j_{0}: j_{1}\right\rangle$, where $j_{0}-i_{0}=j_{1}-i_{1}=h$, such that $I d_{h}(\hat{\imath}, \hat{\jmath})=1$, iff $\hat{\jmath}-\hat{\imath}=h$. We have $I d_{0}=I d$ for any compatible index range. Clearly, an identity or offset indentity matrix can be represented implicitly in constant space and with constant query time. When dealing with identity and offset indentity matrices, we will often omit their index ranges, where they are clear from the context.

A (sub)permutation matrix $P$ over $\left\langle i_{0}: i_{1} \mid j_{0}: j_{1}\right\rangle$ is superdiagonal, if $P(\hat{\imath}, \hat{\jmath})=0$ for all $\hat{\imath}, \hat{\jmath}$, such that $\hat{\imath}<\hat{\jmath}$. In particular, this applies to any offset indentity matrix $I d_{h}$ with $h \geq 0$, as well as to any subpermutation matrix with $i_{1} \leq j_{0}$.

When dealing with (sub)permutation matrices, we will write "nonzeros" for "index pairs corresponding to nonzeros", as long as this does not lead to confusion. We will normally assume that a (sub)permutation matrix with $n$ nonzeros is given implicitly by a compact data structure of size $O(n)$, that allows constant-time access to each nonzero both by the row and by the column index.

Given a permutation matrix $P$ over $(I \mid J)$, and a set $I^{\prime} \subseteq I$, we will denote by $P\left(I^{\prime} \mid \cdot\right)$ the permutation submatrix row-induced by $I^{\prime}$, i.e. the permutation submatrix obtained by deleting from $P$ all columns in $I \backslash I^{\prime}$, and then deleting from the remaining submatrix all zero rows. A columninduced permutation submatrix $P\left(\cdot \mid J^{\prime}\right)$ is defined analogously. Both these operations can be implemented in linear time by a sweep of the nonzeros of matrix $P$.

The following subclasses of Monge matrices play a crucial role in this work.

Definition 6 A square matrix $A$ is called a unit-Monge (respectively, subunitMonge) matrix, if $A^{\square}$ is a permutation (respectively, subpermutation) matrix. Matrix $A$ is called a unit-anti-Monge (respectively, subunit-anti-Monge) matrix, if $-A$ is unit-Monge (respectively, subunit-Monge). 
By definition, any subunit-Monge matrix is unit-Monge, and any unit-Monge matrix is Monge. Similar inclusions hold for (sub)unit-anti-Monge matrices.

Matrices that are both simple and unit-Monge will be our main tool for the rest of this work. Note that such matrices are unit-monotone decreasing (respectively, increasing) in the first (respectively, second) index. Furthermore, a square matrix $A$ is simple unit-Monge, if and only if $A=P^{\Sigma}$, where $P$ is a permutation matrix. The value $A\left(i_{0}, j_{0}\right)=P^{\Sigma}\left(i_{0}, j_{0}\right)$ is the number of (odd half-integer) nonzeros that the (integer) point $\left(i_{0}, j_{0}\right) \lessgtr$-dominates in matrix $P$.

Example 1 The following matrix is simple unit-Monge:

$$
\left[\begin{array}{lll}
0 & 1 & 0 \\
1 & 0 & 0 \\
0 & 0 & 1
\end{array}\right]^{\Sigma}=\left[\begin{array}{llll}
0 & 1 & 2 & 3 \\
0 & 1 & 1 & 2 \\
0 & 0 & 0 & 1 \\
0 & 0 & 0 & 0
\end{array}\right]
$$

A permutation matrix $P$ of size $n$ can be regarded as an implicit representation of the simple unit-Monge matrix $P^{\Sigma}$. An individual element of $P^{\Sigma}$ can be queried in time $O(n)$ by a single sweep of the nonzeros of $P$, counting those that are $\lessgtr$-dominated by the query element. Thinking of the nonzeros of $P$ as odd half-integer points in the plane, this procedure is known as geometric dominance counting.

Existing methods of computational geometry allow us to answer dominance counting queries much more efficiently than by a direct linear sweep, as long as a preprocessing of the point set is allowed.

Theorem 1 Given a (sub)permutation matrix $P$ of size $n$, there exists a data structure which

- has size $O(n \log n)$;

- can be built in time $O(n \log n)$;

- allows to query an individual element of the simple (sub)unit-Monge matrix $P^{\Sigma}$ in time $O\left(\log ^{2} n\right)$;

Proof The required structure is a two-dimensional range tree [15] (see also [93]), built on the set of nonzeros in $P$. There are at most $n$ nonzeros, hence the total number of nodes in the tree is $O(n \log n)$. A dominance counting query on the set of nonzeros can be answered by accessing $O\left(\log ^{2} n\right)$ of the tree nodes.

Using recent results on dominance counting by JaJa et al. [62], the bounds given by Theorem 1 can be improved to size $O(n)$ and individual query time $O\left(\frac{\log n}{\log \log n}\right)$. However, the underlying data structure of Theorem 1 is simpler, requires a less powerful computation model, and is more 
likely to be practical. Therefore, we will be using Theorem 1 as our main technique for implicit representation of simple (sub)unit-Monge matrices.

In addition to ordinary element queries described by Theorem 1, we will also deal with incremental queries, which are given an element of an implicit simple (sub)unit-Monge matrix, and return the value of an adjacent element. This kind of query can be answered directly from the (sub)permutation matrix, without any preprocessing.

Theorem 2 Given a (sub)permutation matrix $P$ of size $n$, and the value $P^{\Sigma}(i, j), i, j \in[0: n]$, the values $P^{\Sigma}(i \pm 1, j), P^{\Sigma}(i, j \pm 1)$, where they exist, can be queried in time $O(1)$.

Proof Let $P$ be a permutation matrix; a generalisation to subpermutation matrices is straightforward. Consider a query of the type $P^{\Sigma}(i+1, j)$; other query types are obtained by symmetry. Let $\hat{\jmath} \in\langle 0: n\rangle$ be such that $P(i+$ $\left.\frac{1}{2}, \hat{\jmath}\right)=1$; value $\hat{\jmath}$ can be obtained from the permutation representation of $P$ in time $O(1)$. We have

$$
P^{\Sigma}(i+1, j)=P^{\Sigma}(i, j)- \begin{cases}1 & \text { if } \hat{\jmath}<j \\ 0 & \text { otherwise }\end{cases}
$$

Incremental queries described by Theorem 2 can be used to answer batch queries, returning a set of elements in a row, column or diagonal of an implicit simple (sub)unit-Monge matrix. In particular, all elements in a given row, column or diagonal of matrix $P^{\Sigma}$ can be obtained by a sequence of incremental queries in time $O(n)$, and a subset of $r$ consecutive elements in time $O\left(r+\log ^{2} n\right)$. 


\section{Chapter 2}

\section{Matrix distance multiplication}

\subsection{Distance multiplication monoids}

We will make extensive use of the (min, + )-semiring on integer or real numbers, where the operators min and + play the role of addition and multiplication, respectively. This semiring is often called distance (or tropical) algebra; for an extensive review of this and related topics, see e.g. Rote [98], Gondran and Minoux [52]. Our techniques are based on matrix-vector and matrix-matrix multiplication in the distance algebra.

Definition 7 Let $A$ be a matrix over $\left[i_{0}: i_{1} \mid j_{0}: j_{1}\right]$. Let $b, c$ be vectors over $\left[j_{0}: j_{1}\right]$ and $\left[i_{0}: i_{1}\right]$ respectively. The matrix-vector distance product $A \odot b=c$ is defined by

$$
c(i)=\min _{j \in\left[j_{0}: j_{1}\right]}(A(i, j)+b(j))
$$

for all $i \in\left[i_{0}: i_{1}\right]$.

Definition 8 Let $A, B, C$ be matrices over $\left[i_{0}: i_{1} \mid j_{0}: j_{1}\right],\left[j_{0}: j_{1} \mid k_{0}: k_{1}\right]$, $\left[i_{0}: i_{1} \mid k_{0}: k_{1}\right]$ respectively. The matrix distance product $A \odot B=C$ is defined by

$$
C(i, k)=\min _{j \in\left[j_{0}: j_{1}\right]}(A(i, j)+B(j, k))
$$

for all $i \in\left[i_{0}: i_{1}\right], k \in\left[k_{0}: k_{1}\right]$.

Like any multiplication of matrices over a semiring, matrix distance multiplication is associative. The set of all square matrices with elements in $[0: \infty]$ over a given index range forms a monoid with respect to distance multiplication. The identity element in this monoid is the matrix

$$
I d_{\odot}(i, j)= \begin{cases}0 & \text { if } i=j \\ +\infty & \text { otherwise }\end{cases}
$$


It is well-known that the set of all Monge matrices is closed under distance multiplication. What is slightly more surprising, but crucial for our method, is that the same is also true for the set of all simple (sub)unit-Monge matrices.

Theorem 3 Let $A, B, C$ be matrices, such that $A \odot B=C$. If $A, B$ are Monge (respectively, simple unit-Monge, simple subunit-Monge), then $C$ is also Monge (respectively, simple unit-Monge, simple subunit-Monge).

Proof Without loss of generality, let $A, B, C$ be over $[0: n \mid 0: n]$.

First, let $A, B$ be Monge matrices. Let $i, i^{\prime}, k, k^{\prime} \in[0: n]$, where $i \leq i^{\prime}$, $k \leq k^{\prime}$. By definition of matrix distance multiplication, we have

$$
\begin{aligned}
& C\left(i, k^{\prime}\right)=\min _{j^{*}}\left(A\left(i, j^{*}\right)+B\left(j^{*}, k^{\prime}\right)\right) \\
& C\left(i^{\prime}, k\right)=\min _{j^{*}}\left(A\left(i^{\prime}, j^{*}\right)+B\left(j^{*}, k\right)\right)
\end{aligned}
$$

Let $j, j^{\prime}$ respectively be the values of $j^{*}$ on which these minima are attained. Suppose $j \leq j^{\prime}$. We have

$$
\begin{array}{lr}
C(i, k)+C\left(i^{\prime}, k^{\prime}\right)= & \text { (definition of } \odot) \\
\min _{j^{*}}\left(A\left(i, j^{*}\right)+B\left(j^{*}, k\right)\right)+\min _{j^{*}}\left(A\left(i^{\prime}, j^{*}\right)+B\left(j^{*}, k^{\prime}\right)\right) \leq & \\
(A(i, j)+B(j, k))+\left(A\left(i^{\prime}, j^{\prime}\right)+B\left(j^{\prime}, k^{\prime}\right)\right)= & (\text { term rearrangement) } \\
\left(A(i, j)+A\left(i^{\prime}, j^{\prime}\right)\right)+\left(B(j, k)+B\left(j^{\prime}, k^{\prime}\right)\right) \leq & (A \text { is Monge) } \\
\left(A\left(i, j^{\prime}\right)+A\left(i^{\prime}, j\right)\right)+\left(B(j, k)+B\left(j^{\prime}, k^{\prime}\right)\right)= & \text { (term rearrangement) } \\
\left(A\left(i, j^{\prime}\right)+B\left(j^{\prime}, k^{\prime}\right)\right)+\left(A\left(i^{\prime}, j\right)+B(j, k)\right)= & \text { (definition of } \left.j, j^{\prime}\right) \\
C\left(i, k^{\prime}\right)+C\left(i^{\prime}, k\right) &
\end{array}
$$

The case $j \geq j^{\prime}$ is treated symmetrically by the Monge property of $B$.

Now, let $A, B$ be simple unit-Monge matrices. We have $A=P_{A}^{\Sigma}, B=$ $P_{B}^{\Sigma}$, where $P_{A}, P_{B}$ are permutation matrices over $\langle 0: n \mid 0: n\rangle$. Clearly, matrices $C$ and $C^{\square}$ are integer. It is also easy to check that matrix $C$ is simple. Furthermore, matrix $C$ is Monge, and therefore $C^{\square}$ is nonnegative.

For any $i \in[0: n]$, we have

$$
\begin{aligned}
& C(i, 0)=\min _{j}\left(P_{A}^{\Sigma}(i, j)+P_{B}^{\Sigma}(j, 0)\right)=\min _{j}\left(P_{A}^{\Sigma}(i, j)+0\right)=0 \\
& C(i, n)=\min _{j}\left(P_{A}^{\Sigma}(i, j)+P_{B}^{\Sigma}(j, n)\right)=\min _{j}\left(P_{A}^{\Sigma}(i, j)+n-j\right)=n-i
\end{aligned}
$$

since the minimum is attained respectively at $j=0$ and $j=n$. Therefore, for all $i \in\langle 0: n\rangle$, we have

$$
\sum_{k} C^{\square}(i, k)=
$$

(definition of $\square$ ) 


$$
\begin{aligned}
& \sum_{k}\left(C\left(i+\frac{1}{2}, k-\frac{1}{2}\right)-C\left(i-\frac{1}{2}, k-\frac{1}{2}\right)-\right. \\
& \left.C\left(i+\frac{1}{2}, k+\frac{1}{2}\right)+C\left(i-\frac{1}{2}, k+\frac{1}{2}\right)\right)=\quad \text { (term cancellation) } \\
& C\left(i+\frac{1}{2}, 0\right)-C\left(i-\frac{1}{2}, 0\right)-C\left(i+\frac{1}{2}, n\right)+C\left(i-\frac{1}{2}, n\right)= \\
& 0-0-\left(n-i-\frac{1}{2}\right)+\left(n-i+\frac{1}{2}\right)=1
\end{aligned}
$$

Symmetrically, for all $k \in\langle 0: n\rangle$, we have

$$
\sum_{i} C^{\square}(i, k)=1
$$

Taken together, the above properties imply that $C^{\square}$ is a permutation matrix, and therefore $C$ is a simple unit-Monge matrix.

Finally, let $A, B$ be simple subunit-Monge matrices. We have $A=P_{A}^{\Sigma}$, $B=P_{B}^{\Sigma}$, where $P_{A}, P_{B}$ are subpermutation matrices over $\langle 0: n| 0$ : $n\rangle$. Let $n-m^{\prime}, n-m^{\prime \prime} \in[0: n]$ be the number of nonzeros in $P_{A}, P_{B}$ respectively. Matrices $P_{A}, P_{B}$ can be extended in a straightforward manner to permutation matrices $\tilde{P}_{A}, \tilde{P}_{B}$ over $\left\langle-m^{\prime} \mid n+m^{\prime}\right\rangle,\left\langle-m^{\prime \prime} \mid n+m^{\prime \prime}\right\rangle$, respectively. Let $m=m^{\prime}+m^{\prime \prime}$. Let $\tilde{P}_{C}$ be a permutation matrix over $\langle-m: n \mid m+n\rangle$, such that

$$
\left(I d_{m^{\prime}} \sqcup \tilde{P}_{A}\right) \sqcup\left(\tilde{P}_{B} \sqcup I d_{m^{\prime \prime}}\right)=\tilde{P}_{C}
$$

We have

$$
\begin{aligned}
& P_{A}=\tilde{P}_{A}\langle 0: n \mid 0: n\rangle \quad P_{B}=\tilde{P}_{B}\langle 0: n \mid 0: n\rangle \\
& P_{C}=P_{A} \boxminus P_{B}=\tilde{P}_{C}\langle 0: n \mid 0: n\rangle
\end{aligned}
$$

The distance multiplication identity matrix $I d \odot$ can be formally considered a Monge matrix (since all indeterminate expressions of the form $+\infty-\infty$ in its density matrix can be considered nonnegative). Therefore, the set of all square Monge matrices forms a submonoid in the distance multiplication monoid of general matrices.

The set of all simple unit-Monge matrices over a given index range also forms a monoid with respect to distance multiplication, but this time with a different identity element:

$$
I d^{\Sigma}(i, j)= \begin{cases}j-i & \text { if } i \leq j \\ 0 & \text { otherwise }\end{cases}
$$

Theorem 3 gives us the basis for performing distance multiplication of simple (sub)unit-Monge matrices implicitly, by taking the density (sub)permutation matrices as input, and producing a density (sub)permutation matrix as output. It will be convenient to introduce special notation for implicit distance multiplication of this kind. 


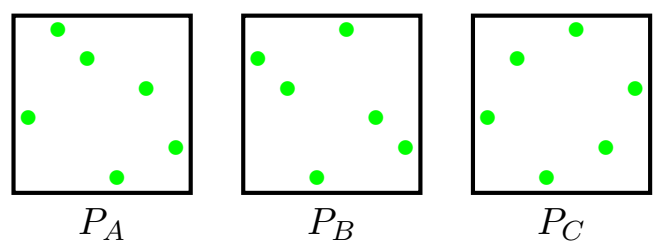

(a) As matrices
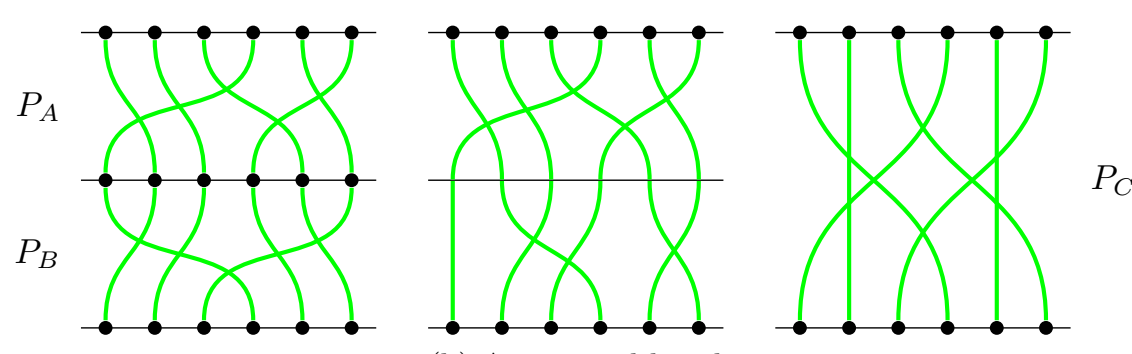

(b) As seaweed braids

Figure 2.1: Matrix distance product $P_{A} \boxminus P_{B}=P_{C}$

Definition 9 Let $P$ be a permutation matrix. Let $b, c$ be vectors. The implicit matrix-vector distance product $P \odot b=c$ is defined by $P^{\Sigma} \odot b=c$.

Definition 10 Let $P_{A}, P_{B}, P_{C}$ be permutation matrices. The implicit matrix distance product $P_{A} \odot P_{B}=P_{C}$ is defined by $P_{A}^{\Sigma} \odot P_{B}^{\Sigma}=P_{C}^{\Sigma}$.

Example 2 In Figure 2.1, Subfigure 2.1a shows a triple of $6 \times 6$ permutation matrices $P_{A}, P_{B}, P_{C}$, with nonzeros indicated by green ${ }^{1}$ bullets, such that $P_{A} \triangleright P_{B}=P_{C}$.

\subsection{Seaweed braids}

Further understanding of the distance multiplication monoid of simple unitMonge matrices can be gained by the following construction. Given a permutation matrix $P$ over $(I \mid J)$, we represent the indices in sets $I$ and $J$ by nodes on two parallel lines, respecting the order of indices within each set. We represent every nonzero $P(\hat{\imath}, \hat{\jmath})=1$ by connecting node $\hat{\imath} \in I$ with node $\hat{\jmath} \in J$ by a continuous strictly monotone line called a seaweed. We call the resulting configuration a seaweed braid. Unless $P$ is the identity matrix $I d$, some of the seaweeds in the seaweed braid will have to cross. However, due to monotonicity of seaweeds, no "unnecessary" crossings can occur; in other words, a given pair of seaweeds can only cross at most once.

Consider the implicit distance product $P_{A} \odot P_{B}=P_{C}$, where $P_{A}, P_{B}$, $P_{C}$ are permutation matrices over $(I \mid J),(J \mid K)$ and $(I \mid K)$, respectively.

\footnotetext{
${ }^{1}$ For colour illustrations, the reader is referred to the online version of this work. If the colour version is not available, all references to colour can be ignored.
} 
We represent the indices in sets $I, J, K$ by nodes on three parallel lines, and the nonzeros of the input matrices $P_{A}, P_{B}$ by two sets of seaweeds connecting the corresponding nodes. The seaweed braid representation of the output matrix $P_{C}$ can be obtained as follows. First, we remove the nodes representing the index set $J$. At each removed node $\hat{\jmath} \in J$, the two adjacent seaweeds, which represent nonzeros $P_{A}(\hat{\imath}, \hat{\jmath})=1$ and $P_{B}(\hat{\jmath}, \hat{k})=1$ for some $\hat{\imath}, \hat{k}$, are joined together. We now have a seaweed configuration between nodes of $I$ and nodes of $K$. However, some seaweed pairs in this configuration may cross twice. We now "comb" the seaweeds by running through all their crossings, respecting the top-to-bottom partial order of the crossings. Every time, we check whether the two crossing seaweeds already have a previous crossing above the current one. If this is the case, then we undo the current crossing by cutting it out of the configuration, and replacing it by two non-crossing seaweed pieces. After all the crossings have been processed, the resulting configuration is a seaweed braid representing the output matrix $P_{C}$.

Example 3 In Figure 2.2, Subfigure 2.1b shows the seaweed braids representing the implicit matrix distance product in Subfigure 2.1a.

Seaweed braids can be formalised algebraically as follows. The seaweed monoid $\mathcal{T}_{n}$ is a finitely presented monoid on $n$ generators $i d, g_{1}, g_{2}, \ldots, g_{n-1}$. Generator $i d$ is the identity element, which corresponds to a seaweed braid where all the seaweeds are parallel. Each of the remaining generators $g_{t}$ corresponds to a seaweed braid where all the seaweeds are parallel, except a pair of neighbouring seaweeds in positions $t-\frac{1}{2}$ and $t+\frac{1}{2}$, which do cross. In matrix notation, the identity generator $i d$ corresponds to the simple unit-Monge matrix $I d^{\Sigma}$, and each generator $g_{t}$ corresponds to a simple unit-Monge matrix $P_{t}^{\Sigma}$, where an elementary transposition matrix $P_{t}$ is a permutation matrix defined by $P_{t}(\hat{\imath}, \hat{\jmath})=1$ iff $\hat{\imath}=\hat{\jmath} \notin\left\{t-\frac{1}{2}, t+\frac{1}{2}\right\}$ or $\{\hat{\imath}, \hat{\jmath}\}=$ $\left\{t-\frac{1}{2}, t+\frac{1}{2}\right\}$. Concatenation of words in the generators corresponds to the composition of seaweed braids. The presentation of monoid $\mathcal{T}_{n}$ consists of the idempotence relations

$$
g_{t}^{2}=g_{t} \quad t \in[1: n-1]
$$

and the braid relations

$$
\begin{array}{ll}
g_{t} g_{u}=g_{u} g_{t} & t, u \in[1: n-1], u-t \geq 2 \\
g_{t} g_{u} g_{t}=g_{u} g_{t} g_{u} & t, u \in[1: n-1], u-t=1
\end{array}
$$

Intuitively, relations (2.1) express the seaweeds' double crossing property; relations (2.2) express the commutativity of independent seaweed crossings (note that pairs of crossing with $|t-u| \leq 1$ are not independent, so their 
corresponding generators do not commute); and relations (2.3) give two equivalent expressions for a local crossing of three seaweeds.

We are now able to establish a formal connection between distance multiplication of simple unit-Monge matrices and the seaweed monoid.

Theorem 4 The distance multiplication monoid of $n \times n$ simple unit-Monge matrices is isomorphic to the seaweed monoid $\mathcal{T}_{n}$.

Proof It is straightforward to check that any simple unit-Monge matrix $P^{\Sigma}$ can be decomposed into a distance product of matrices $P_{t}^{\Sigma}$ for various values of $t$; this can be visualised as drawing a seaweed configuration for $P$, and decomposing it into individual seaweed crossings. Hence, matrices $P_{t}^{\Sigma}$ serve as generators for the distance multiplication monoid of simple unitMonge matrices. By using the defining relations of the seaweed monoid, it is also straightforward to check that multiplication in both monoids agrees on the generators. By associativity of multiplication, this implies that multiplication in monoids agrees on all the elements, therefore the two monoids are isomorphic.

The classical positive braid monoid (see e.g. [65, Section 6.5]) on generators $i d, g_{1}, g_{2}, \ldots, g_{n-1}$, is defined by the braid relations (2.2)-(2.3) alone. Therefore, the seaweed monoid is isomorphic to the quotient of the positive braid monoid by the idempotence relations (2.1). A generalisation of the seaweed monoid is given by Coxeter monoids, which arise naturally as subgroup monoids in groups. The theory of Coxeter monoids can be traced back to Bourbaki [20], and has been developed by Tsaranov [111] and Richardson and Springer [96]. The contents of this and the following sections can be regarded as the first step in the algorithmic study of Coxeter monoids.

\subsection{Distance multiplication algorithms}

In this section, we show that distance multiplication of Monge and simple unit-Monge matrices can be performed much more efficiently than a naive implementation of the definitions. One of the ingredients in our method is the classical algorithm by Aggarwal et al. [1] (see also [49]), which allows efficient searching in totally monotone matrices.

Lemma 1 ([1]) Let $A$ be an $n \times n$ implicit totally monotone matrix, where each element can be queried in time $q$. Then the problem of finding the minimum element in every row of $A$ (the row minima problem) can be solved in time $O(q n)$.

Our method is based on efficient matrix distance multiplication, exploiting the special properties of the matrices. We begin with matrix-vector distance multiplication. For generic, explicitly represented matrices, the 
fastest method for matrix-vector distance multiplication of size $n$ is by direct application of Definition 7 in time $O\left(n^{2}\right)$. For implicit Monge matrices, the running time can be substantially reduced.

Theorem 5 Let $A$ be an $n \times n$ implicit Monge matrix, where each element can be queried in time q. Let $b, c$ be n-vectors, such that $A \odot b=c$. Given vector $b$, vector $c$ can be computed in time and memory $O(q n)$.

Proof Let $\tilde{A}(i, j)=A(i, j)+b(j)$. It easy to check that matrix $\tilde{A}$ is a Monge matrix. Clearly, each element of $\tilde{A}$ can be queried in time $q+O(1)$. The problem of computing the product $A \odot b=c$ is equivalent to the row minima problem in matrix $\tilde{A}$, which can be solved in time (and therefore also memory) $O(q n)$ by Lemma 1 .

Applying the above theorem to implicit unit-Monge matrices represented by Theorem 1 with $q=O\left(\log ^{2} n\right)$, we obtain immediately an algorithm for implicit matrix-vector distance multiplication, running in time $O\left(n \log ^{2} n\right)$. Using batch queries based on Theorem 2, this running time can be improved to $O(n \log n)$ as follows.

Theorem 6 Let $P$ be an $n \times n$ (sub)permutation matrix. Let $b, c$ be $n$ vectors, such that $P \square b=c$. Given the nonzeros of $P$ and the full vector $b$, vector $c$ can be computed in time $O(n \log n)$ and memory $O(n)$.

Proof As in the proof of Theorem 5, we let $\tilde{A}(i, j)=P^{\Sigma}(i, j)+b(j)$. We then use the standard algorithm of [1] for finding row minima in a monotone (but not necessarily totally monotone) matrix. First, we obtain all elements in row $n / 2$ of matrix $\tilde{A}$ by a row batch query for matrix $P^{\Sigma}$ via Theorem 2, and compute the minimum element in this row. We are now faced with two subproblems of finding the row minima in the submatrix of $\tilde{A}$ with $i<n / 2$ (respectively, $i>n / 2$ ). By using the monotonicity property of matrix $\tilde{A}$, each of the subproblems can be reduced to an instance of the original problem of size $n / 2$. Both the batch query and the problem reduction run in time and memory $O(n)$, therefore the total running time is $O\left(n+2 \cdot n / 2+2^{2} \cdot n / 2^{2}+\ldots\right)=O(n \log n)$ and the total memory is $O(n)$.

We now consider matrix-matrix distance multiplication. For generic matrices, direct application of Definition 8 gives an algorithm for matrix distance multiplication of size $n$, running in time $O\left(n^{3}\right)$. Slightly subcubic algorithms for this problem have also been obtained. The fastest currently known algorithm is by Chan [26], running in time $O\left(\frac{n^{3}(\log \log n)^{3}}{\log ^{2} n}\right)$.

For Monge matrices, distance multiplication can be easily performed in quadratic time.

Theorem 7 Let $A, B, C$ be $n \times n$ matrices, such that $A$ is Monge, and $A \odot B=C$. Given matrices $A, B$, matrix $C$ can be computed in time and memory $O\left(n^{2}\right)$. 
Proof The problem of computing the product $A \odot B=C$ is equivalent to $n$ instances of the matrix-vector product $A \odot b=c$, where $b$ (respectively, $c$ ) is a column of $B$ (respectively, $C$ ). Every one of these instances can be solved in time $O(n)$ by Theorem 5, so the overall running time (and therefore also memory) is $n \cdot O(n)=O\left(n^{2}\right)$.

When matrices are represented explicitly, the running time in Theorem 7 is clearly optimal. However, for implicit simple unit-Monge matrices, the distance multiplication time can be reduced even further. In $[105,108]$, we gave an algorithm running in time $O\left(n^{1.5}\right)$. We now show that still further improvement is possible.

Theorem 8 Let $P_{A}, P_{B}, P_{C}$ be $n \times n$ (sub)permutation matrices, such that $P_{A} \triangleright P_{B}=P_{C}$. Given the nonzeros of $P_{A}, P_{B}$, the nonzeros of $P_{C}$ can be computed in time $O(n \log n)$.

Proof Without loss of generality, let $P_{A}, P_{B}, P_{C}$ be permutation matrices over $\langle 0: n \mid 0: n\rangle$. A generalisation to subpermutation matrices is as in Theorem 3.

The algorithm is defined by recursion on $n$.

Recursion base. If $n=1$, the computation is trivial.

Recursive step. Assume without loss of generality that $n>1$ is even. Informally, the idea is to split the range of index $j$ in the definition of matrix distance product (Definition 8) into two subranges of size $\frac{n}{2}$. For each of these subranges of $j$, we use the sparsity of the input permutation matrix $P_{A}$ (respectively, $P_{B}$ ) to partition the range of index $i$ (respectively, $k$ ) into two disjoint, not necessarily contiguous, subsets of size $\frac{n}{2}$. We then call the algorithm recursively on the two resulting half-sized subproblems, and use the two returned half-sized permutation matrices to reconstruct the output permutation matrix $P_{C}$, relying on the Monge properties of the respective distribution matrices.

We now describe the recursive step in more detail. We have

$$
P_{A}^{\Sigma} \odot P_{B}^{\Sigma}=P_{C}^{\Sigma}
$$

Let

$$
\begin{array}{lll}
P_{A, l o}=P_{A}\left\langle * \mid 0: \frac{n}{2}\right\rangle & P_{B, l o}=P_{B}\left\langle 0: \frac{n}{2} \mid *\right\rangle & P_{A, l o}^{\Sigma} \odot P_{B, l o}^{\Sigma}=P_{C, l o}^{\Sigma} \\
P_{A, h i}=P_{A}\left\langle * \mid \frac{n}{2}: n\right\rangle & P_{B, h i}=P_{B}\left\langle\frac{n}{2}: n \mid *\right\rangle & P_{A, h i}^{\Sigma} \odot P_{B, h i}^{\Sigma}=P_{C, h i}^{\Sigma}
\end{array}
$$

In the first subproblem, matrices $P_{A, l o}, P_{B, l o}$ are rectangular (respectively, $n \times \frac{n}{2}$ and $\left.\frac{n}{2} \times n\right)$ subpermutation matrices, each with $\frac{n}{2}$ nonzeros. It is easy to see that a zero row (respectively, column) in $P_{A, l o}, P_{B, l o}$ corresponds to a zero row (respectively, column) in their implicit distance product $P_{C, l o}$. Therefore, we can delete all zero rows and columns from $P_{A, l o}, P_{B, l o}, P_{C, l o}$, 
obtaining, after appropriate index remapping, three $\frac{n}{2} \times \frac{n}{2}$ permutation matrices. Consequently, the first subproblem can be solved by first performing a linear-time index remapping (corresponding to the deletion of zero rows and columns from $P_{A, l o}, P_{B, l o}$ ), then making a recursive call on the resulting half-sized problem, and then performing an inverse index remapping (corresponding to the reinsertion of the zero rows and columns into $\left.P_{C, l_{o}}\right)$. The second subproblem is solved analogously.

Since the nonzeros in the two subproblems have disjoint index ranges, the sum $P_{C, l o}+P_{C, h i}$ is an $n \times n$ permutation matrix. We have

$$
\begin{aligned}
& P_{C}^{\Sigma}(i, k)=\min _{j \in[0: n]}\left(P_{A}^{\Sigma}(i, j)+P_{B}^{\Sigma}(j, k)\right)= \\
& \min \left(\min _{j \in\left[0: \frac{n}{2}\right]}\left(P_{A}^{\Sigma}(i, j)+P_{B}^{\Sigma}(j, k)\right), \min _{j \in\left[\frac{n}{2}: n\right]}\left(P_{A}^{\Sigma}(i, j)+P_{B}^{\Sigma}(j, k)\right)\right)
\end{aligned}
$$

for all $i, k \in[0: n]$. The first argument in the above expression can now be rewritten as

$$
\begin{aligned}
& \min _{j \in\left[0: \frac{n}{2}\right]}\left(P_{A}^{\Sigma}(i, j)+P_{B}^{\Sigma}(j, k)\right)= \\
& \min _{j \in\left[0: \frac{n}{2}\right]}\left(P_{A, l o}^{\Sigma}(i, j)+P_{B, l o}^{\Sigma}(j, k)+P_{B, h i}^{\Sigma}\left(\frac{n}{2}, k\right)\right)= \\
& \min _{j \in\left[0: \frac{n}{2}\right]}\left(P_{A, l o}^{\Sigma}(i, j)+P_{B, l o}^{\Sigma}(j, k)\right)+P_{B, h i}^{\Sigma}\left(\frac{n}{2}, k\right)= \\
& P_{C, l_{o}}^{\Sigma}(i, k)+P_{C, h i}^{\Sigma}(0, k)
\end{aligned}
$$

The second argument is rewritten analogously, so we have

$$
P_{C}^{\Sigma}(i, k)=\min \left(P_{C, l_{o}}^{\Sigma}(i, k)+P_{C, h i}^{\Sigma}(0, k), P_{C, h i}^{\Sigma}(i, k)+P_{C, l o}^{\Sigma}(i, n)\right)
$$

for all $i, k \in[0: n]$. In order to compute the nonzeros of matrix $P_{C}$ efficiently, consider the difference of arguments in the above expression:

$$
\begin{aligned}
& \delta(i, k)= \\
& \left(P_{C, l_{o}}^{\Sigma}(i, k)+P_{C, h i}^{\Sigma}(0, k)\right)-\left(P_{C, h i}^{\Sigma}(i, k)+P_{C, l_{o}}^{\Sigma}(i, n)\right)= \\
& \left(P_{C, h i}^{\Sigma}(0, k)-P_{C, h i}^{\Sigma}(i, k)\right)-\left(P_{C, l o}^{\Sigma}(i, n)-P_{C, l_{o}}^{\Sigma}(i, k)\right)= \\
& \sum_{\hat{\imath} \in\langle 0: i\rangle, \hat{k} \in\langle 0: k\rangle} P_{C, h i}(\hat{\imath}, \hat{k})-\sum_{\hat{\imath} \in\langle i: n\rangle, \hat{k} \in\langle k: n\rangle} P_{C, l_{o}}(\hat{\imath}, \hat{k})
\end{aligned}
$$

Since $P_{C, l o}, P_{C, h i}$ are subpermutation matrices, and $P_{C, l o}+P_{C, h i}$ a permutation matrix, it follows that function $\delta$ is unit-monotone increasing in each of its arguments.

The sign of function $\delta$ plays an important role in determining the positions of nonzeros in $P_{C}$. Let us fix some $\hat{\imath}, \hat{k} \in\langle 0: n\rangle$, and consider the four values $\delta\left(\hat{\imath} \pm \frac{1}{2}, \hat{k} \pm \frac{1}{2}\right)$. Three cases are possible. 
Case $\delta\left(\hat{\imath}+\frac{1}{2}, \hat{k}+\frac{1}{2}\right) \leq 0$. By monotonicity of $\delta$, we have $\delta\left(\hat{\imath} \pm \frac{1}{2}, \hat{k} \pm \frac{1}{2}\right) \leq 0$ for all four sign combinations. Therefore,

$$
P_{C}^{\Sigma}\left(\hat{\imath} \pm \frac{1}{2}, \hat{k} \pm \frac{1}{2}\right)=P_{C, l_{o}}^{\Sigma}\left(\hat{\imath} \pm \frac{1}{2}, \hat{k} \pm \frac{1}{2}\right)
$$

for all four sign combinations chosen consistently on both sides of the equation. Hence, $P_{C}(\hat{\imath}, \hat{k})=P_{C, l o}(\hat{\imath}, \hat{k})$.

Case $\delta\left(\hat{\imath}-\frac{1}{2}, \hat{k}-\frac{1}{2}\right) \geq 0$. Symmetrically, we have $P_{C}(\hat{\imath}, \hat{k})=P_{C, h i}(\hat{\imath}, \hat{k})$.

Case $\delta\left(\hat{\imath}-\frac{1}{2}, \hat{k}-\frac{1}{2}\right)<0$ and $\delta\left(\hat{\imath}+\frac{1}{2}, \hat{k}+\frac{1}{2}\right)>0$. By monotonicity of $\delta$, we have $\delta\left(\hat{\imath}-\frac{1}{2}, \hat{k}+\frac{1}{2}\right)=\delta\left(\hat{\imath}+\frac{1}{2}, \hat{k}-\frac{1}{2}\right)=0$. Therefore,

$$
P_{C}^{\Sigma}\left(\hat{\imath}+\frac{1}{2}, \hat{k}+\frac{1}{2}\right)=P_{C, h i}^{\Sigma}\left(\hat{\imath}+\frac{1}{2}, \hat{k}+\frac{1}{2}\right)<P_{C, l o}^{\Sigma}\left(\hat{\imath}+\frac{1}{2}, \hat{k}+\frac{1}{2}\right)
$$

and, furthermore,

$$
P_{C}^{\Sigma}\left(\hat{\imath} \pm \frac{1}{2}, \hat{k} \pm \frac{1}{2}\right)=P_{C, l_{o}}^{\Sigma}\left(\hat{\imath} \pm \frac{1}{2}, \hat{k} \pm \frac{1}{2}\right)
$$

for the remaining three sign combinations chosen consistently on both sides of the equation. By Definitions 1, 2, we have

$$
\begin{aligned}
& P_{C}(\hat{\imath}, \hat{k})=\cdots-P_{C}^{\Sigma}\left(\hat{\imath}+\frac{1}{2}, \hat{k}+\frac{1}{2}\right)+\cdots \\
& P_{C, l o}(\hat{\imath}, \hat{k})=\cdots-P_{C, l_{o}}^{\Sigma}\left(\hat{\imath}+\frac{1}{2}, \hat{k}+\frac{1}{2}\right)+\cdots
\end{aligned}
$$

By (2.4), we have a strict inequality between the two terms shown, and by (2.5), the omitted terms are pairwise equal between the two expressions. Hence, $P_{C}(\hat{\imath}, \hat{k})>P_{C, l o}(\hat{\imath}, \hat{k})$. Since both $P_{C}, P_{C, l o}$ are zero-one matrices, this implies that $P_{C}(\hat{\imath}, \hat{k})=1$ and $P_{C, l o}(\hat{\imath}, \hat{k})=0$ (symmetrically, also $\left.P_{C, h i}(\hat{\imath}, \hat{k})=0\right)$.

Summarising the above three cases, we have $P_{C}(\hat{\imath}, \hat{k})=1$, if and only if one of the following mutually exclusive conditions holds:

$$
\begin{aligned}
& P_{C, l o}(\hat{\imath}, \hat{k})=1 \text { and } \delta\left(\hat{\imath}+\frac{1}{2}, \hat{k}+\frac{1}{2}\right) \leq 0 \\
& P_{C, h i}(\hat{\imath}, \hat{k})=1 \text { and } \delta\left(\hat{\imath}-\frac{1}{2}, \hat{k}-\frac{1}{2}\right) \geq 0 \\
& \delta\left(\hat{\imath}-\frac{1}{2}, \hat{k}-\frac{1}{2}\right)<0 \text { and } \delta\left(\hat{\imath}+\frac{1}{2}, \hat{k}+\frac{1}{2}\right)>0
\end{aligned}
$$

In order to perform the checks (2.6)-(2.8) efficiently, it is sufficient to find for each $d \in[-n+1: n-1]$ a value $r(d) \in[1: 2 n-1]$, such that $r(d)+d$ is odd, and

$$
\begin{array}{ll}
\delta(i, k) \leq 0 & \text { if } i+k<r(k-i) \\
\delta(i, k) \geq 0 & \text { if } i+k>r(k-i)
\end{array}
$$

for all $i, k \in[0: n]$ (note that the above list of two cases is exhaustive, since $r(k-i)-(i+k)=r(k-i)+(k-i)-2 k$ must be odd, and therefore 
$i+k \neq r(k-i))$. Such a value $r(d)$ is guaranteed to exist by the monotonicity of function $\delta$. Furthermore, values $r(d)$ can be chosen so that $\mid r\left(\hat{d}+\frac{1}{2}\right)-$ $r\left(\hat{d}-\frac{1}{2}\right) \mid=1$ for all $\hat{d} \in\langle-n+1: n-1\rangle$. Informally, array $r$ defines a monotone rectilinear path, consisting of points $\left(\frac{r(d)-d}{2}, \frac{r(d)+d}{2}\right)$, from the bottom-left to the top-right corner of $\langle 0: n\rangle^{2}$.

By definition of array $r$, for each $d$ we have

$$
\begin{aligned}
& w^{-}(d)=\delta\left(\frac{r(d)-d-1}{2}, \frac{r(d)+d-1}{2}\right) \in[-1,0] \\
& w^{+}(d)=\delta\left(\frac{r(d)-d+1}{2}, \frac{r(d)+d+1}{2}\right) \in[0,1]
\end{aligned}
$$

We call the values $w^{-}(d), w^{+}(d)$ witnesses for $r(d)$.

Array $r$ can be computed efficiently as follows. We loop from $d=-n+1$ to $d=n-1$. For each $d$, we obtain the value $r(d)$ along with its two witnesses.

Initially, we have $d=-n+1, r(-n+1)=n$; the witnesses $w^{-}(-n+1)=$ $\delta(n-1,0)$ and $w^{+}(-n+1)=\delta(n, 1)$ can be easily computed in time $O(1)$.

Now assume that for a current value of $d$, we have the value $r(d)$, and the witnesses $w^{-}(d), w^{+}(d)$. Our next goal is to compute $r(d+1)$, along with its two witnesses. Let

$$
w^{*}=\delta\left(\frac{r(d)-d-1}{2}, \frac{r(d)+d+1}{2}\right) \in[-1: 1]
$$

Value $w^{*}$ can be obtained from either $w^{-}(d)$ or $w^{+}(d)$ by Theorem 2 in time $O(1)$. We now let

$$
r(d+1)=r(d)+ \begin{cases}1 & \text { if } w^{*} \in[-1: 0] \\ -1 & \text { if } w^{*} \in[0: 1]\end{cases}
$$

If $w^{*}=0$, then the choice between 1 and -1 is made arbitrarily. Following this choice, we obtain the new witnesses as

$$
\begin{aligned}
& w^{-}(d+1)= \begin{cases}\delta\left(\frac{r(d)-d-3}{2}, \frac{r(d)+d-1}{2}\right) & \text { if } w^{*} \in[-1: 0] \\
w^{*} & \text { if } w^{*} \in[0: 1]\end{cases} \\
& w^{+}(d+1)= \begin{cases}w^{*} & \text { if } w^{*} \in[-1: 0] \\
\delta\left(\frac{r(d)-d+1}{2}, \frac{r(d)+d+3}{2}\right) & \text { if } w^{*} \in[0: 1]\end{cases}
\end{aligned}
$$

In each case, the value for the new witness can be obtained from respectively $w^{-}(d), w^{+}(d)$ by Theorem 2 in time $O(1)$. If $w^{*}=0$, then the choices are made consistently with the arbitrary choice made in the definition of $r(d+1)$.

The described loop runs until $d=n-1$. At this point, we necessarily have $r(n-1)=n, w^{-}(n-1)=\delta(0, n-1)$ and $w^{+}(n-1)=\delta(1, n)$. The whole loop runs in time $O(n)$.

Given arrays $r, w^{-}, w^{+}$, conditions (2.6)-(2.8) can now be expressed as follows:

$$
P_{C, l o}(\hat{\imath}, \hat{k})=1 \text { and } \hat{\imath}+\hat{k}<r(\hat{k}-\hat{\imath})
$$




$$
\begin{aligned}
& P_{C, h i}(\hat{\imath}, \hat{k})=1 \text { and } \hat{\imath}+\hat{k}>r(\hat{k}-\hat{\imath}) \\
& \hat{\imath}+\hat{k}=r(\hat{k}-\hat{\imath}) \text { and } w^{-}(\hat{k}-\hat{\imath})=-1 \text { and } w^{+}(\hat{k}-\hat{\imath})=1
\end{aligned}
$$

The nonzeros of $P_{C}$ satisfying either of the conditions (2.9), (2.10) can be found in time $O(n)$ by checking directly each of the nonzeros in matrices $P_{C, l o}$ and $P_{C, h i}$. The nonzeros of $P_{C}$ satisfying condition (2.11) can be found in time $O(n)$ by a linear sweep of the values $r(d)$ for all $d \in[-n+1: n-1]$. For each $d$, we let $\hat{\imath}=\frac{r(d)+d}{2}, \hat{k}=\frac{r(d)-d}{2}$, and substitute these values into (2.11). We have now obtained all the nonzeros of matrix $P_{C}$.

End of recursive step.

Time analysis. The recursion tree is a balanced binary tree of height $\log n$. In the root node, the computation runs in time $O(n)$. In each subsequent level, the number of nodes doubles, and the running time per node decreases by a factor of 2 . Therefore, the overall running time is $O(n \log n)$.

Example 4 Figure 2.2 illustrates the proof of Theorem 8 on a problem instance with a solution generated by the Mathematica 7 software. Subfigure 2.2a shows a pair of input $20 \times 20$ permutation matrices $P_{A}, P_{B}$, with nonzeros indicated by green bullets. Subfigure $2.2 \mathrm{~b}$ shows the partitioning of the implicit $20 \times 20$ matrix distance multiplication problem into two $10 \times 10$ subproblems. The nonzeros in the two subproblems are shown respectively by red crosses and blue diamonds. Subfigure 2.2c shows a recursive step. The boundaries separating sets $\delta^{-1}([-10:-1]), \delta^{-1}(\{0\}), \delta^{-1}([1: 10])$ are indicated by the red and the blue line. Function $r$ corresponds to an arbitrary monotone rectilinear path within $\delta^{-1}(\{0\})$, inclusive of the boundaries. In particular, either of the boundaries itself can be taken to define $r$. The nonzeros in the output matrix $P_{C}$ satisfying $(2.9),(2.10),(2.11)$ are indicated respectively by red crosses, blue diamonds and green bullets; note that overall, there are 20 such nonzeros, and that they define a permutation matrix.

\subsection{Technical lemmas}

In this section, we prove two technical lemmas required by the applications presented in the following chapters.

Lemma 2 Let $P_{A}, P_{B}, P_{C}$ be permutation matrices over $\left\langle i_{0}: i_{1} \mid j_{0}: j_{1}\right\rangle$, $\left\langle j_{0}: j_{1} \mid j_{0}: j_{1}\right\rangle,\left\langle i_{0}: i_{1} \mid j_{0}: j_{1}\right\rangle$ respectively, such that

$$
\begin{aligned}
& P_{B}=I d \sqsupset P_{B}\left\langle j_{0}^{\prime}: j_{1}^{\prime} \mid j_{0}^{\prime}: j_{1}^{\prime}\right\rangle \\
& P_{A} \boxminus P_{B}=P_{C}
\end{aligned}
$$

where $j_{0}^{\prime}, j_{1}^{\prime} \in\left[j_{0}: j_{1}\right]$. Let $n=i_{1}-i_{0}=j_{1}-j_{0}, m=j_{1}^{\prime}-j_{0}^{\prime}$. Given nonzeros of matrices $P_{A}, P_{B}$ stored in main memory, matrix $P_{C}$ can be obtained in main memory in time $O(m \log m)$. 


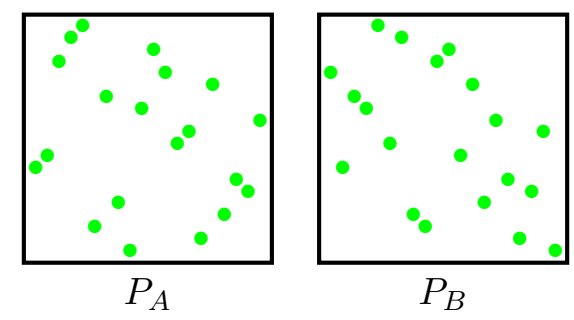

(a) Input matrices $P_{A}, P_{B}$

$$
P_{B, l o}, P_{B, h i}
$$
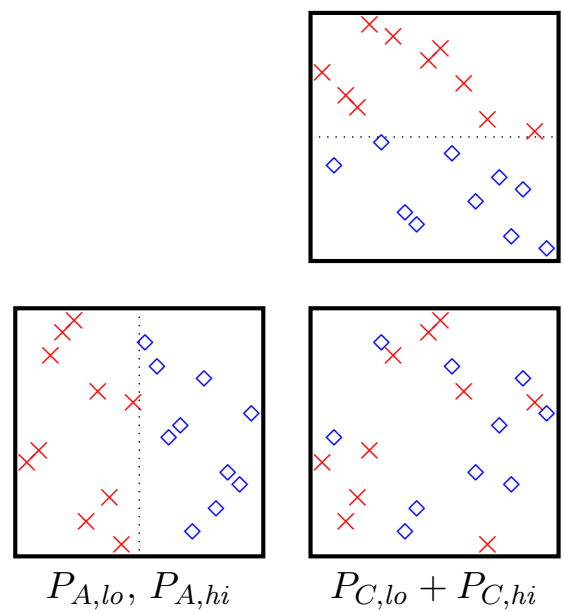

(b) Subproblems $P_{A, l o} \boxminus P_{B, l o}=P_{C, l o}$ and $P_{A, h i} \boxminus P_{B, h i}=P_{C, h i}$

$$
P_{B, l o}, P_{B, h i}
$$
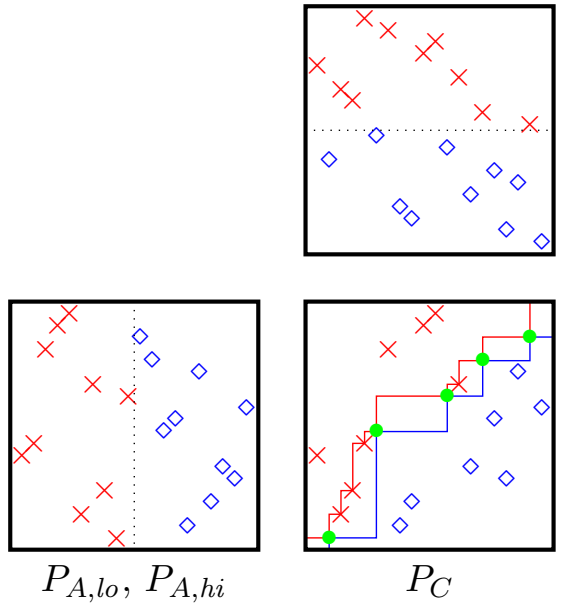

(c) Conversion of $P_{C, l o}+P_{C, h i}$ into $P_{C}$

Figure 2.2: Proof of Theorem 8: $P_{A} \boxminus P_{B}=P_{C}$ 


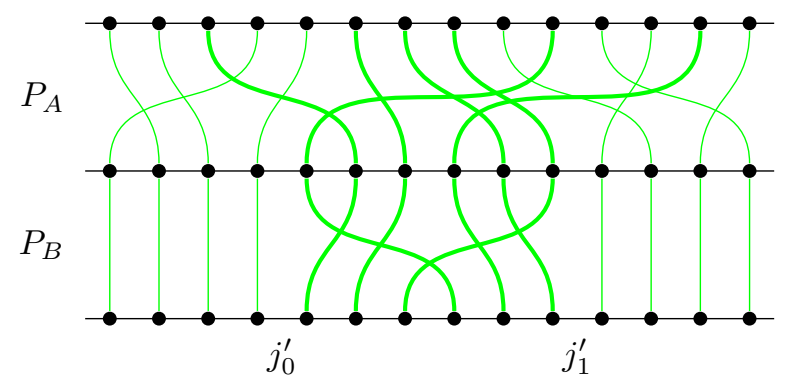

(a) Input matrices $P_{A}, P_{B}$

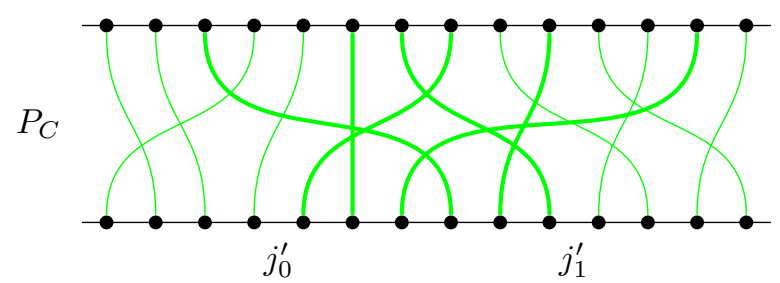

(b) Output matrix $P_{C}$

Figure 2.3: Statement of Lemma 2: $P_{A} \boxminus P_{B}=P_{C}$

Proof Let

$$
\begin{aligned}
& \tilde{P}_{A} \leftarrow P_{A}\left\langle\cdot \mid j_{0}^{\prime}: j_{1}^{\prime}\right\rangle \quad \tilde{P}_{B} \leftarrow P_{B}\left\langle j_{0}^{\prime}: j_{1}^{\prime} \mid j_{0}^{\prime}: j_{1}^{\prime}\right\rangle \\
& \tilde{P}_{C} \leftarrow \tilde{P}_{A} \sqcup \tilde{P}_{B} \\
& P_{C} \leftarrow P_{A}\left\langle\cdot \mid j_{0}: j_{0}^{\prime}\right\rangle \sqcup \tilde{P}_{C} \sqcup P_{A}\left\langle\cdot \mid j_{1}^{\prime}: j_{1}\right\rangle
\end{aligned}
$$

All index operations are performed in time $O(m)$. Implicit matrix distance multiplication is performed in time $O(m \log m)$ by Theorem 8 . The final assignment can be performed in time $O(m)$ by partially overwriting nonzeros of matrix $P_{A}$ with nonzeros of matrix $P_{C}$ in main memory. The overall running time is $O(m \log m)$.

Example 5 Figure 2.3 illustrates the statement of Lemma 2. Three horizontal lines represent respectively the index ranges of $i, j, k$. The nonzeros in $P_{A}, P_{B}, P_{C}$ are shown by seaweeds; the thick seaweeds correspond to the nonzeros involved in the matrix distance multiplication, and the thin seaweeds to the remaining nonzeros.

Lemma 3 Let $P$ be a superdiagonal (sub)permutation matrix over $\langle-m$ : $n|n: m+n\rangle$, where $\frac{n}{m}$ is integer. There exist (sub)permutation matrices $P_{0}, P_{1}, \ldots, P_{\frac{n}{m}-1}$ over $\langle-m: n \mid-m: n\rangle$, such that

$$
P_{s}=I d \sqsupset P_{s}\langle(s-1) m:(s+1) m \mid(s-1) m:(s+1) m\rangle
$$

for all $s \in\left\{0,1, \ldots, \frac{n}{m}-1\right\}$, and

$$
P=P_{0} \boxminus P_{1} \boxminus \cdots \boxminus P_{\frac{n}{m}-1} \boxminus I d_{m}
$$




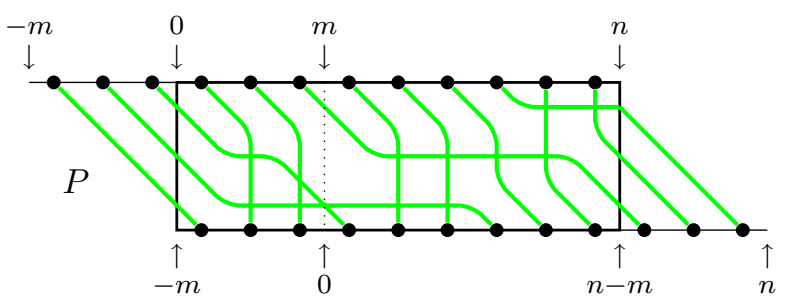

(a) Adjusted input matrix $P \boxminus I d_{-m}$

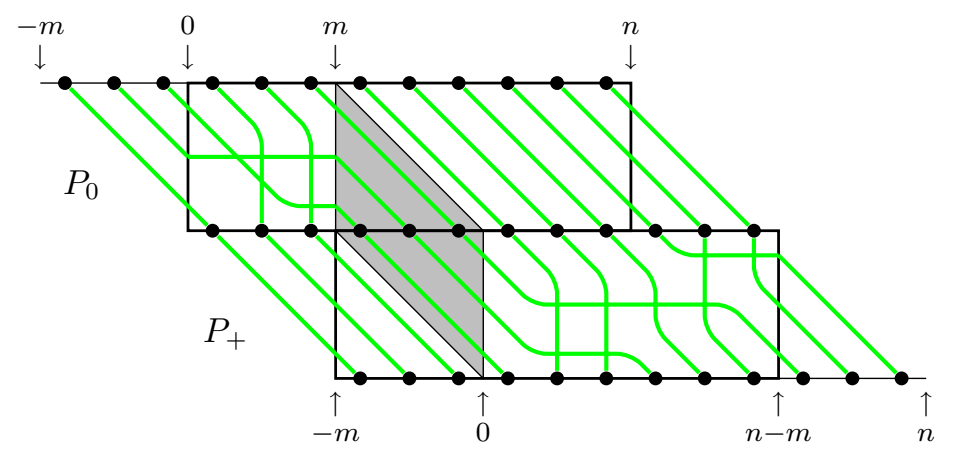

(b) Decomposition $P_{0} \boxminus P_{+}$

Figure 2.4: Proof of Lemma 3: $P \boxminus I d_{-m}=P_{0} \bullet P_{+}$

Given the nonzeros of $P$, the non-trivial nonzeros of all of $P_{0}, P_{1}, \ldots, P_{\frac{n}{m}-1}$ can be computed in time $O(n)$.

Proof We begin by multiplying both sides of the equation by $I d_{-m}$ :

$$
P \boxminus I d_{-m}=P_{0} \boxminus P_{1} \boxminus \cdots \boxminus P_{\frac{n}{m}-1}
$$

The required decomposition of the adjusted input matrix $\tilde{P} \square I d_{-m}$ is defined recursively as follows.

Recursion base. If $n=m$, the decomposition is trivial: $\tilde{P}=P_{0}$.

Recursive step. We partition matrix $\tilde{P}$ as

$$
\tilde{P}=\tilde{P}\langle-m: m \mid \cdot\rangle \sqcup \tilde{P}\langle m: n \mid \cdot\rangle
$$

Since matrix $P$ is superdiagonal, we have

$$
\tilde{P}\langle-m: m \mid \cdot\rangle=\tilde{P}\left\langle-m: m \mid-m: 0, \hat{t}_{\frac{1}{2}}, \ldots, \hat{t}_{m-\frac{1}{2}}\right\rangle
$$

for some $\hat{t}_{\frac{1}{2}}, \ldots, \hat{t}_{m-\frac{1}{2}} \in\langle 0: n\rangle, \hat{t}_{\frac{1}{2}}<\ldots<\hat{t}_{m-\frac{1}{2}}$. We now define

$$
P_{0}(\hat{\imath}, \hat{\jmath})= \begin{cases}\tilde{P}(\hat{\imath}, \hat{\jmath}) & \hat{\imath} \in\langle-m: m\rangle, \hat{\jmath} \in\langle-m: 0\rangle \\ \tilde{P}\left(\hat{\imath}, \hat{t}_{\hat{\jmath}}\right) & \hat{\imath} \in\langle-m: m\rangle, \hat{\jmath} \in\langle 0: m\rangle \\ \operatorname{Id}(\hat{\imath}, \hat{\jmath}) & \hat{\imath} \in\langle m: n\rangle, \hat{\jmath} \in\langle m: n\rangle\end{cases}
$$




$$
P_{+}(\hat{\jmath}, \hat{k})= \begin{cases}I d(\hat{\jmath}, \hat{k}) & \hat{\jmath} \in\langle-m: 0\rangle, \hat{k} \in\langle-m: 0\rangle \\ I d\left(\hat{t}_{\hat{\jmath}}, \hat{k}\right) & \hat{\jmath} \in\langle 0: m\rangle, \hat{k} \in\langle 0: n\rangle \\ \tilde{P}(\hat{\jmath}, \hat{k}) & \hat{\jmath} \in\langle m: n\rangle, \hat{k} \in\langle 0: n\rangle\end{cases}
$$

Intuitively, each nonzero $\tilde{P}(\hat{\imath}, \hat{\jmath})$ within the submatrix $\tilde{P}\langle-m: m \mid-m: 0\rangle$ is preserved as $P_{0}(\hat{\imath}, \hat{\jmath})$; each nonzero $\tilde{P}(\hat{\jmath}, \hat{k})$ within the submatrix $\tilde{P}\langle m$ : $n|0: n\rangle$ is preserved as $P_{+}(\hat{\jmath}+m, \hat{k}+m)$. The remaining nonzeros of $\tilde{P}$ are within the submatrix $\tilde{P}\langle-m: m \mid 0: n\rangle$; each of these nonzeros $\tilde{P}(\hat{\imath}, \hat{k})$ is preserved as a pair of nonzeros $P_{0}(\hat{\imath}, \hat{t}), P_{+}(\hat{t}, \hat{k})$; the intermediate index $\hat{t} \in\langle 0: m\rangle$ is chosen so that

- nonzeros $P_{0}(\hat{\imath}, \hat{t})$ preserve the $\lessgtr$-dominance order of the corresponding nonzeros in $P$;

- nonzeros $P_{+}(\hat{t}, \hat{k})$ form a $\lessgtr$-antichain.

By construction, we have $\tilde{P}=P_{0} \triangleright P_{+}$. The decomposition is then applied recursively to matrix $P_{+}$.

End of recursive step.

Time analysis. Each recursive step can be implemented in time $O(m)$, therefore the full decomposition can be obtained in time $O\left(m \cdot \frac{n}{m}\right)=O(n)$.

Example 6 Figure 2.4 illustrates the statement of Lemma 3. The $m \times n$ rectangle corresponding to the core of $P$ is split into an $m \times m$ square corresponding to the core of $P_{0}$, and an $m \times(n-m)$ rectangle corresponding to the core of $P_{+}$. The resulting rectangles are arranged vertically with a shift by $m$. The seaweeds that do not cross the partition between the rectangles are preserved by the construction. The seaweeds that do cross the partition line are also preserved, by passing them through a parallelogramshaped "buffer zone". Note the latter class of seaweeds are all uncrossed in $P_{0}$, and keep their original crossings from $P$ in $P_{+}$. 


\section{Chapter 3}

\section{Semi-local string comparison}

\subsection{Semi-local LCS}

We will consider strings of characters taken from an alphabet. No a priori assumptions are made on the size of the alphabet and on the model of computation; we will make specific assumptions in different contexts (e.g. a fixed finite alphabet with only equality comparisons, or an alphabet of integers up to a given $n$ with standard arithmetic operations, etc.) Two alphabet characters $\alpha, \beta$ match, if $\alpha=\beta$, and mismatch otherwise. In addition to alphabet characters, we introduce the special non-alphabet character ' $\$$ ', which only matches itself and does not match any alphabet characters, and the wildcard character ' $\diamond$ ', which matches both itself and all the alphabet characters.

It will be convenient to index strings by odd half-integer, rather than integer indices, e.g. string $a=\alpha_{\frac{1}{2}} \alpha_{\frac{3}{2}} \ldots \alpha_{m-\frac{1}{2}}$. We will index strings as vectors, writing e.g. $a(\hat{\imath})=\alpha_{\hat{\imath}}, a\langle i: j\rangle=\alpha_{i+\frac{1}{2}} \ldots \alpha_{j-\frac{1}{2}}$. String concatenation will be denoted by juxtaposition.

Given a string, we distinguish between its contiguous substrings, and not necessarily contiguous subsequences. Special cases of a substring are a prefix and a suffix of a string. Given a string $a$ of length $m$, we use the take/drop notation of [113] for prefixes and suffixes of $a$ :

$$
\begin{array}{ll}
a \nmid k=a\langle 0: k\rangle & a\rfloor k=a\langle k: m\rangle \\
a \nmid k=a\langle m-k: m\rangle & a\lfloor k=a\langle 0: m-k\rangle
\end{array}
$$

Unless indicated otherwise, our algorithms will take as input a string $a$ of length $m$, and a string $b$ of length $n$.

One of the simplest form of string comparison is the (global) subsequence recognition problem. Given a text string $t$ of length $n$ and a pattern string $p$ of length $m \leq n$, the problem asks whether $t$ contains the whole of $p$ as a subsequence. This problem has been considered e.g. by Aho et al. [2] as the 
"subsequence matching problem"; they describe a straightforward algorithm running in time $O(m)$.

A classical method of string comparison is based on the following definition.

Definition 11 Given strings $a, b$, the longest common subsequence (LCS) problem asks for the length of the longest string that is a subsequence of both $a$ and $b$. We will call this length the LCS score of strings $a, b$.

Example 7 The LCS score of strings $a=$ "baabcbca", $b=$ "baabcabcabaca" is 8 ; string $b$ contains the whole string $a$ as a subsequence. The LCS score of string $a$ against substring $b\langle 4: 11\rangle=$ "cabcaba" is 5 ; this score is realised by a common subsequence "abcba". This example, which will serve as a running example for this chapter, is borrowed from Alves et al. [7].

The subsequence recognition problem is equivalent to asking whether the LCS score of $t$ against $p$ is exactly $m$. The general LCS problem is more computationally demanding than subsequence recognition; the best known algorithms run within logarithmic factors of $O(m n)$. We will recall the necessary background on LCS algorithms in Sections 4.1, 5.1, 8.3.

The string comparison method adopted by this work generalises the LCS problem as follows.

Definition 12 Given strings $a, b$, the semi-local LCS problem asks for the LCS scores as follows:

- $a$ against every substring of $b$ (the string-substring LCS scores);

- every prefix of $a$ against every suffix of $b$ (the prefix-suffix LCS scores);

- symmetrically, the substring-string LCS scores and the suffix-prefix $L C S$ scores, defined as above but with the roles of $a$ and $b$ exchanged. $\square$

A traditional distinction, especially in computational biology, is between global (full string against full string) and local (all substrings against all substrings) comparison. Our problem lies in between, hence the term "semilocal". Many string comparison algorithms output either a single optimal comparison score across all local comparisons, or a number of local comparison scores that are "sufficiently close" to the globally optimal. In contrast with this approach, Definition 12 requires to output all the locally optimal comparison scores. It turns out that this is a very natural and useful generalisation of the LCS problem.

The semi-local LCS problem includes the LCS problem as a special case. We will consider semi-local LCS algorithms on plain strings in Sections 4.1, 5.1, and on compressed strings in Section 8.3.

Another special case of the semi-local LCS problem is the local subsequence recognition problem, which asks for the substrings in $a$ containing $b$ 
as a subsequence. This problem can also be regarded as a basic form of approximate pattern matching. We will consider algorithms for local subsequence recognition and other types of approximate pattern matching on plain strings in Section 5.4, and on compressed strings in Section 8.4.

In certain contexts, e.g. when one input string is much longer than the other, we may not be able to solve all four components of the semi-local LCS problem efficiently. In such cases, we may wish to settle for the following restriction.

Definition 13 Given strings $a, b$, the three-way semi-local LCS problem asks for the string-substring, prefix-suffix and suffix-prefix LCS scores as in Definition 12, but excludes the substring-string LCS scores. The same term three-way semi-local LCS problem will also refer to the symmetric version of the problem, that asks for the prefix-suffix, suffix-prefix and substring-string LCS scores, but excludes the string-substring LCS scores.

We will occasionally use the term full semi-local LCS to distinguish the standard four-way semi-local LCS problem from its three-way restriction.

\subsection{Alignment dags and highest-score matrices}

It is well-known that an instance of the LCS problem can be represented by a dag (directed acyclic graph) on a rectangular grid of nodes, where character matches correspond to edges scoring 1 , and mismatches to edges scoring 0 .

Definition 14 An alignment dag is a weighted dag, defined on the set of nodes $v_{l, i}, l \in\left[l_{0}: l_{1}\right], i \in\left[i_{0}, i_{1}\right]$. The edge and path weights are called scores. For all $l \in\left[l_{0}: l_{1}\right], \hat{l} \in\left\langle l_{0}: l_{1}\right\rangle, i \in\left[i_{0}, i_{1}\right], \hat{\imath} \in\left\langle i_{0}: i_{1}\right\rangle$, the alignment dag contains:

- the horizontal edge $v_{l, \hat{\imath}-\frac{1}{2}} \rightarrow v_{l, \hat{\imath}+\frac{1}{2}}$ and the vertical edge $v_{\hat{l}-\frac{1}{2}, i} \rightarrow$ $v_{\hat{l}+\frac{1}{2}, i}$, both with score 0 ;

- the diagonal edge $v_{\hat{l}-\frac{1}{2}, \hat{\imath}-\frac{1}{2}} \rightarrow v_{\hat{l}+\frac{1}{2}, \hat{\imath}+\frac{1}{2}}$ with score either 0 or 1 .

An alignment dag can be viewed as an $\left(l_{1}-l_{0}\right) \times\left(i_{1}-i_{0}\right)$ grid of cells. An instance of the semi-local LCS problem on strings $a, b$ corresponds to an $m \times n$ alignment dag $G_{a, b}$; a cell indexed by $\hat{l} \in\langle 0: m\rangle, \hat{\imath} \in\langle 0: n\rangle$ is called a match cell, if $a(\hat{l})$ matches $b(\hat{\imath})$, and a mismatch cell otherwise (recall that the strings may contain wildcard characters). The diagonal edges in match cells have score 1 , and in mismatch cells score 0 . Clearly, the diagonal edges with score 0 do not affect maximum node-to-node scores, and can therefore be ignored.

Particular examples of an alignment dag are the full-mismatch dag and the full-match dag, which consist entirely of mismatch or match cells, respectively. The dag $G_{a, b}$ is the full-mismatch dag, when the strings $a, b$ 


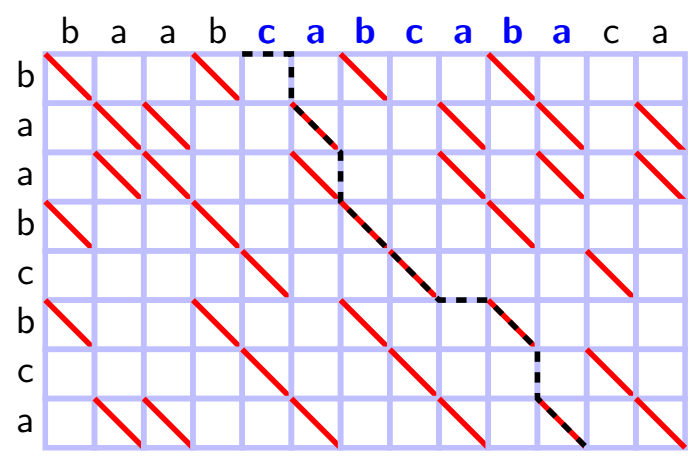

Figure 3.1: Alignment dag $G_{a, b}$ and a highest-scoring path

have no characters in common. The dag $G_{a, b}$ is the full-match dag, when both strings consist of a single repeated character, or when one of the strings consists entirely of wildcard characters.

Given an instance of the LCS problem on strings $a, b$, common stringsubstring, suffix-prefix, prefix-suffix, and substring-string subsequences correspond, respectively, to paths of the following form in the alignment dag $G_{a, b}$ :

$$
v_{0, i} \rightsquigarrow v_{m, i^{\prime}} \quad v_{l, 0} \rightsquigarrow v_{m, i^{\prime}} \quad v_{0, i} \rightsquigarrow v_{l^{\prime}, n} \quad v_{l, 0} \rightsquigarrow v_{l^{\prime}, n}
$$

where $l, l^{\prime} \in[0: m], i, i^{\prime} \in[0: n]$. The length of each subsequence is equal to the total score of its corresponding path. The semi-local LCS problem is equivalent to the problem of finding the highest-scoring paths for each of the four path types (3.1) and each possible pair of endpoints.

Example 8 Figure 3.1 shows the alignment dag for strings $a=$ "baabcbca", $b=$ "baabcabcabaca". The highlighted path of score 5 corresponds to the string-substring LCS score for string $a$ against substring $b\langle 4: 11\rangle=$ "cabcaba".

Finding the highest-scoring paths is equivalent to finding the corresponding shortest distances in an undirected graph, obtained from the alignment dag by assigning length 1 to vertical and horizontal edges, assigning lengths 0 and 2 to diagonal edges in match and mismatch cells respectively, and ignoring edge directions. Thus, the problem is equivalent to the problem of finding distances between boundary nodes and all nodes on a special case of a weighted undirected planar graph. This problem has been previously studied by Schmidt [101] on real-weighted alignment dags, and by Klein [68] and Cabello and Chambers [23] on general real-weighted undirected planar graphs. In contrast with these approaches, we exploit both the special structure of the alignment dag, and the discreteness of the weights.

The analysis of the four path types (3.1) can be reduced to a single type, by padding one of the input strings with wildcards. Accordingly, we need to 


\begin{tabular}{c|c|c|c|c|c|c|c|c|c|c|c|c|c}
0 & 1 & 2 & 3 & 4 & 5 & 6 & 6 & 7 & 8 & 8 & 8 & 8 & 8 \\
\hline-1 & 0 & 1 & 2 & 3 & 4 & 5 & 5 & 6 & 7 & 7 & 7 & 7 & 7 \\
\hline-2 & -1 & 0 & 1 & 2 & 3 & 4 & 4 & 5 & 6 & 6 & 6 & 6 & 7 \\
\hline-3 & -2 & -1 & 0 & 1 & 2 & 3 & 3 & 4 & 5 & 5 & 6 & 6 & 7 \\
\hline-4 & -3 & -2 & -1 & 0 & 1 & 2 & 2 & 3 & 4 & 4 & 5 & 5 & 6 \\
\hline-5 & -4 & -3 & -2 & -1 & 0 & 1 & 2 & 3 & 4 & 4 & 5 & 5 & 6 \\
\hline-6 & -5 & -4 & -3 & -2 & -1 & 0 & 1 & 2 & 3 & 3 & 4 & 4 & 5 \\
\hline-7 & -6 & -5 & -4 & -3 & -2 & -1 & 0 & 1 & 2 & 2 & 3 & 3 & 4 \\
\hline-8 & -7 & -6 & -5 & -4 & -3 & -2 & -1 & 0 & 1 & 2 & 3 & 3 & 4 \\
\hline-9 & -8 & -7 & -6 & -5 & -4 & -3 & -2 & -1 & 0 & 1 & 2 & 3 & 4 \\
\hline$-10-9$ & -8 & -7 & -6 & -5 & -4 & -3 & -2 & -1 & 0 & 1 & 2 & 3 \\
\hline$-11-10-9$ & -8 & -7 & -6 & -5 & -4 & -3 & -2 & -1 & 0 & 1 & 2 \\
\hline$-12-11-10-9$ & -8 & -7 & -6 & -5 & -4 & -3 & -2 & -1 & 0 & 1 \\
\hline$-13-12-11-10-9$ & -8 & -7 & -6 & -5 & -4 & -3 & -2 & -1 & 0
\end{tabular}

Figure 3.2: Submatrices $H_{a, b}[0: 13 \mid 0: 13]$ and $P_{a, b}\langle 0: 13 \mid 0: 13\rangle$

consider an extended alignment dag for string $a$ over $\langle 0: m\rangle$ against string $\diamond^{m} b \diamond^{m}$ over $\langle-m: m+n\rangle$.

Definition 15 Given strings $a, b$, the corresponding semi-local score matrix ${ }^{1}$ is a matrix over $[-m: n \mid 0: m+n]$, defined by

$$
H_{a, b}(i, j)=\max \operatorname{score}\left(v_{0, i} \rightsquigarrow v_{m, j}\right)
$$

where $i \in[-m: n], j \in[0: m+n]$, and the maximum is taken across all paths between the given endpoints in the $m \times(2 m+n)$ alignment dag $G_{a, \diamond m} b \diamond^{m}$. If $i=j$, we have $H_{a, b}(i, j)=0$. By convention, if $j<i$, then we let $H_{a, b}(i, j)=j-i<0$.

Example 9 Figure 3.2 shows the submatrix $H_{a, b}[0: 13 \mid 0: 13]$, giving all string-substring LCS scores for strings $a, b$ as in the previous examples. The entry $H_{a, b}(4,11)=5$ is circled.

The solution for each of the four components (3.1) of the semi-local LCS problem can now be obtained from the semi-local score matrix $H_{a, b}$ as follows:

$$
\begin{aligned}
& \max \operatorname{score}\left(v_{0, j} \rightsquigarrow v_{m, j^{\prime}}\right)=H_{a, b}\left(j, j^{\prime}\right) \\
& \max \operatorname{score}\left(v_{i, 0} \rightsquigarrow v_{m, j^{\prime}}\right)=H_{a, b}\left(-i, j^{\prime}\right)-i \\
& \max \operatorname{score}\left(v_{0, j} \rightsquigarrow v_{i^{\prime}, n}\right)=H_{a, b}\left(j, m+n-i^{\prime}\right)-m+i^{\prime}
\end{aligned}
$$

\footnotetext{
${ }^{1}$ These matrices are called "DIST matrices" e.g. in [101, 33], and "score matrices" in [106]. Our current terminology is chosen to reflect the semi-local score-maximising nature of the matrix elements, while avoiding confusion with pairwise substitution score matrices used in comparative genomics (see e.g. [63]).
} 


$$
\max \operatorname{score}\left(v_{i, 0} \rightsquigarrow v_{i^{\prime}, n}\right)=H_{a, b}\left(-i, m+n-i^{\prime}\right)-m-i+i^{\prime}
$$

where $i, i^{\prime} \in[0: m], j, j^{\prime} \in[0: n]$, and the maxima are taken across all paths between the given endpoints.

Special properties of semi-local score matrices have been extensively used in algorithm design. These properties are captured by the following theorem.

Theorem 9 Given strings $a, b$, the corresponding semi-local score matrix $H_{a, b}$ is unit-anti-Monge. In particular, we have

$$
H_{a, b}(i, j)=j-i-P_{a, b}^{\Sigma}(i, j)
$$

where $P_{a, b}$ is a superdiagonal permutation matrix over $\langle-m: n \mid 0: m+n\rangle_{\text {. }}$

Proof Let $\hat{\imath} \in\langle-m: n\rangle, \hat{\jmath} \in\langle 0: m+n\rangle$. Any crossing pair of paths $v_{0, \hat{\imath}+\frac{1}{2}} \rightsquigarrow v_{m, \hat{\jmath}-\frac{1}{2}}$ and $v_{0, \hat{\imath}-\frac{1}{2}} \rightsquigarrow v_{m, \hat{\jmath}+\frac{1}{2}}$ can be rearranged into a non-crossing pair of paths $v_{0, \hat{\imath}-\frac{1}{2}} \rightsquigarrow v_{m, \hat{\jmath}-\frac{1}{2}}$ and $v_{0, \hat{\imath}+\frac{1}{2}} \rightsquigarrow v_{m, \hat{\jmath}+\frac{1}{2}}$ of the same total score. Therefore, we have $H_{a, b}^{\square}(\hat{\imath}, \hat{\jmath}) \leq 0$, hence matrix $H_{a, b}$ is anti-Monge, and matrix $P_{a, b}^{\Sigma}$ is Monge. Furthermore, we have

$$
\begin{aligned}
& P_{a, b}^{\Sigma}\left(\hat{\imath}-\frac{1}{2}, m+n\right)-P_{a, b}^{\Sigma}\left(\hat{\imath}+\frac{1}{2}, m+n\right)= \\
& \quad 1-H_{a, b}\left(\hat{\imath}-\frac{1}{2}, m+n\right)+H_{a, b}\left(\hat{\imath}+\frac{1}{2}, m+n\right)=1-m+m=1
\end{aligned}
$$

and

$$
\begin{aligned}
& P_{a, b}^{\Sigma}\left(-m, \hat{\jmath}+\frac{1}{2}\right)-P_{a, b}^{\Sigma}\left(-m, \hat{\jmath}-\frac{1}{2}\right)= \\
& \quad 1-H_{a, b}\left(-m, \hat{\jmath}+\frac{1}{2}\right)+H_{a, b}\left(-m, \hat{\jmath}-\frac{1}{2}\right)=1-m+m=1
\end{aligned}
$$

Together, the above properties imply that $P_{a, b}^{\Sigma}$ is a simple unit-Monge matrix, so $P_{a, b}$ is a permutation matrix.

In fact, Theorem 9 holds more generally for any alignment dag, not necessarily defined by any particular pair of strings. However, the given form of the theorem will be sufficient for the rest of this work.

The key idea of our approach is to regard the semi-local score matrix $H_{a, b}$ as implicitly represented by the permutation matrix $P_{a, b}$.

Definition 16 Given strings $a, b$, the semi-local seaweed matrix is a permutation matrix $P_{a, b}$ over $\langle-m: n \mid 0: m+n\rangle$, defined by Theorem 9 .

The nonzeros of matrix $P_{a, b}$ can be regarded as an implicit solution to the semi-local LCS problem.

Example 10 Figure 3.2 shows the unit-anti-Monge property of matrix $H_{a, b}$ by a coloured grid pattern, where the red (respectively, blue) lines separate 


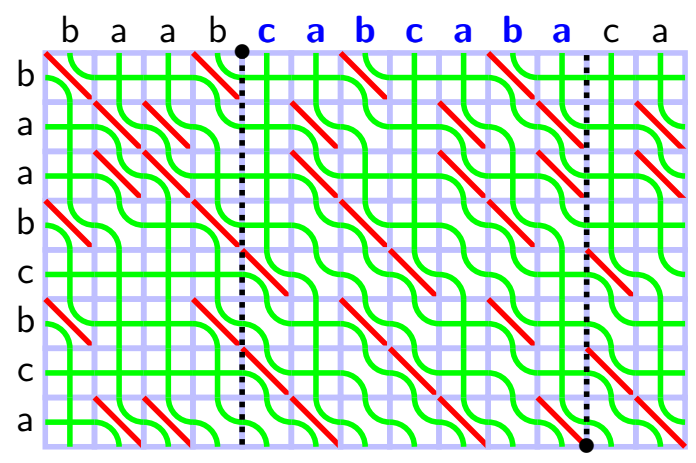

Figure 3.3: Alignment dag $G_{a, b}$ and nonzeros of $P_{a, b}$ as seaweeds

matrix elements that differ by 1 (respectively, by 0 ). The nonzeros of the subpermutation submatrix $P_{a, b}\langle 0: 13 \mid 0: 13\rangle$ are shown by green bullets.

Figure 3.3 shows the semi-local seaweed matrix $P_{a, b}$ over $\langle-8: 13| 0$ : $8+13\rangle$ as a seaweed braid, laid out directly on the alignment $\operatorname{dag} G_{a, b}$. The nonzeros correspond to seaweeds, laid out as paths in the dual graph. In particular, every nonzero $P_{a, b}(\hat{\imath}, \hat{\jmath})=1$, where $\hat{\imath}, \hat{\jmath} \in\langle 0: 13\rangle$, is represented by a seaweed originating between the nodes $v_{0, \hat{\imath}-\frac{1}{2}}$ and $v_{0, \hat{\imath}+\frac{1}{2}}$, and terminating between the nodes $v_{8, \hat{\jmath}-\frac{1}{2}}$ and $v_{8, \hat{\jmath}+\frac{1}{2}}$. The remaining seaweeds, originating or terminating at the sides of the dag, correspond to nonzeros $P_{a, b}(\hat{\imath}, \hat{\jmath})=1$, where either $\hat{\imath} \in\langle-8: 0\rangle$ or $\hat{\jmath} \in\langle 13: 8+13\rangle$ (or both). In particular, every nonzero $P_{a, b}(\hat{\imath}, \hat{\jmath})=1$, where $\hat{\imath} \in\langle-8: 0\rangle$ (respectively, $\hat{\jmath} \in\langle 13$ : $8+13\rangle)$ is represented by a seaweed originating between the nodes $v_{-\hat{\imath}-\frac{1}{2}, 0}$ and $v_{-\hat{\imath}+\frac{1}{2}, 0}$ (respectively, terminating between the nodes $v_{8+13-\hat{\jmath}-\frac{1}{2}, 13}$ and $\left.v_{8+13-\hat{\jmath}+\frac{1}{2}, 13}\right)$. For the purposes of this example, the specific layout of the seaweeds between their endpoints is not important. However, this layout will become meaningful in the context of the algorithms described in the next chapter.

In Figure 3.2, the nonzeros of $P_{a, b}\langle 0: 13 \mid 0: 13\rangle$ that are $\lessgtr$-dominated by the entry $P_{a, b}^{\Sigma}(4,11)=2$ correspond to the green bullets lying below and to the left of the circled entry. In Figure 3.3, these $\lessgtr$-dominated nonzeros correspond to seaweeds fitting completely between the two vertical lines $i=4$ and $j=11$. Note that in both cases, there are exactly two $\lessgtr$ dominated nonzeros, and that $H_{a, b}(4,11)=11-4-2=5$. The full set of nonzeros in the semi-local seaweed matrix $P_{a, b}$ corresponds in Figure 3.3 to the full set of $8+13=21$ seaweeds.

With minimal modification, the definitions of score and seaweed matrices can also be applied individually to the string-substring (symmetrically, substring-string), and the three-way semi-local LCS problems. For that, we split up the definitions as follows. 
Definition 17 Given strings $a, b$, the corresponding string-substring, prefixsuffix, suffix-prefix and substring-string score matrices are respectively the following submatrices of the semi-local score matrix $H_{a, b}$ over $\langle-m: n| 0$ : $m+n\rangle$ :

$$
\begin{aligned}
& H_{a, b}^{s-s u b}=H_{a, b}\langle 0: n \mid 0: n\rangle \\
& H_{a, b}^{p x-s x}=H_{a, b}\langle 0: n \mid n: m+n\rangle \\
& H_{a, b}^{s x-p x}=H_{a, b}\langle-m: 0 \mid 0: n\rangle \\
& H_{a, b}^{s u b-s}=H_{a, b}\langle-m: 0 \mid n: m+n\rangle
\end{aligned}
$$

Definition 18 Given strings $a, b$, the corresponding string-substring, prefixsuffix, suffix-prefix and substring-string seaweed matrices are respectively the following subpermutation submatrices of the semi-local seaweed matrix $P_{a, b}$ over $\langle-m: n \mid 0: m+n\rangle$ :

$$
\begin{aligned}
& P_{a, b}^{s-s u b}=P_{a, b}\langle 0: n \mid 0: n\rangle \\
& P_{a, b}^{p x-s x}=P_{a, b}\langle 0: n \mid n: m+n\rangle \\
& P_{a, b}^{s x-p x}=P_{a, b}\langle-m: 0 \mid 0: n\rangle \\
& P_{a, b}^{s u b-s}=P_{a, b}\langle-m: 0 \mid n: m+n\rangle
\end{aligned}
$$

Example 11 Matrices shown in Figure 3.2 are, in the above notation, matrices $H_{a, b}^{s-s u b}=H_{a, b}[0: 13 \mid 0: 13]$ and $P_{a, b}^{s-s u b}=P_{a, b}[0: 13 \mid 0: 13]$.

The nonzeros of each matrix introduced in Definition 18 can be regarded as an implicit solution to the corresponding component of the semi-local LCS problem. In particular, the first three matrices can serve as an implicit solution to the three-way semi-local LCS problem. The combined number of nonzeros in these three matrices is at least $n$ and at most $\min (m+n, 2 n)$; note that for $m \geq n$, this number is independent of $m$.

Both the full semi-local seaweed matrices and their submatrices introduced in Definition 18 can be processed into an efficient data structure of Theorem 1 for answering individual semi-local score queries.

The definitions of score and seaweed matrices are not symmetric with respect to the order of the input matrices. The precise relationship between score matrices $H_{a, b}, H_{b, a}$, as well as their seaweed counterparts, is given by the following lemma.

Lemma 4 Given input strings $a, b$, we have

$$
H_{b, a}(i, j)=H_{a, b}(-i, m+n-j)-i+j-n
$$

for all $i \in[0: n], j \in[0: m]$, and

$$
P_{b, a}(\hat{\imath}, \hat{\jmath})=P_{a, b}(-\hat{\imath}, m+n-\hat{\jmath})
$$

for all $\hat{\imath} \in\langle 0: n\rangle, \hat{\jmath} \in\langle 0: m\rangle$.

Proof Straightforward by definitions. 


\subsection{Score matrix composition}

We now describe how the techniques of previous sections can be applied within a divide-and-conquer framework. Let $a^{\prime}, a^{\prime \prime}, b^{\prime}, b^{\prime \prime}$ be strings of length $m^{\prime}, m^{\prime \prime}, n^{\prime}, n^{\prime \prime}$ respectively.

Given a string $a=a^{\prime} a^{\prime \prime}$, a substring $a\left\langle i^{\prime}: i^{\prime \prime}\right\rangle$ with $i^{\prime} \in\left\langle 0: m^{\prime}\right\rangle, i^{\prime \prime} \in\left\langle m^{\prime}\right.$ : $m\rangle$ will be called an overlap substring; in other words, an overlap substring of $a$ is a concatenation of a suffix of $a^{\prime}$ and a prefix of $a^{\prime \prime}$.

Definition 19 Given strings $a^{\prime}, a^{\prime \prime}, b$, the overlap submatrix of the substringstring seaweed matrix $P_{a^{\prime} a^{\prime \prime}, b}^{s u b-s}$ is the subpermutation matrix

$$
P_{a^{\prime}, a^{\prime \prime}, b}^{o s u b-s}=P_{a^{\prime} a^{\prime \prime}, b}^{s u b-s}\left\langle-m^{\prime}: 0 \mid n: m^{\prime \prime}+n\right\rangle
$$

Symmetrically, the overlap submatrix of the string-substring seaweed matrix $P_{a, b^{\prime} b^{\prime \prime}}^{s-s u b}$ is the subpermutation matrix

$$
P_{a, b^{\prime}, b^{\prime \prime}}^{s-o s b}=P_{a, b^{\prime} b^{\prime \prime}}^{s-s u b}\left\langle 0: n^{\prime} \mid n^{\prime}: n\right\rangle
$$

Note that, in contrast with Definition 18, the dimensions of matrices introduced by Definition 19 depend on the lengths of individual component strings in the concatenation $\left(a^{\prime}, a^{\prime \prime}\right.$ or $b^{\prime}, b^{\prime}$, respectively). Hence, the notation for these matrices involves three string subscripts.

Without lost of generality, we will consider the semi-local LCS problem for a concatenation string $a=a^{\prime} a^{\prime \prime}$ against a fixed string $b$. The equivalent results for a fixed string $a$ against a concatenation string $b^{\prime} b^{\prime \prime}$ will follow as corollaries by symmetry.

The alignment dag $G_{a, b}$ consists of alignment subdags $G_{a^{\prime}, b}, G_{a^{\prime \prime}, b}$, sharing a horizontal row of $n$ nodes and $n-1$ edges, which is simultaneously the bottom row of $G_{a^{\prime}, b}$ and the top row of $G_{a^{\prime \prime}, b}$. We will say that dag $G_{a, b}$ is the composite of dags $G_{a^{\prime}, b}$ and $G_{a^{\prime \prime}, b}$.

Our goal is, given the semi-local score matrices $H_{a^{\prime}, b}, H_{a^{\prime \prime}, b}$, to compute matrix $H_{a, b}$ efficiently. We call this procedure score matrix composition.

By Theorem 9, score matrices $H_{a^{\prime}, b}, H_{a^{\prime \prime}, b}, H_{a, b}$ can be represented implicitly by seaweed permutation matrices $P_{a^{\prime}, b}, P_{a^{\prime \prime}, b}, P_{a, b}$. We argue that score matrix composition corresponds generally to implicit distance multiplication of these seaweed matrices.

Example 12 Figure 3.4 shows an instance of semi-local score matrix composition represented by seaweed matrices. The nonzeros in the matrices are given by seaweeds. Since in this case there is no explicit underlying alignment dag, the layout of every seaweed should be regarded as completely arbitrary (except for disallowing double crossings).

We first consider the string-substring LCS problem. 


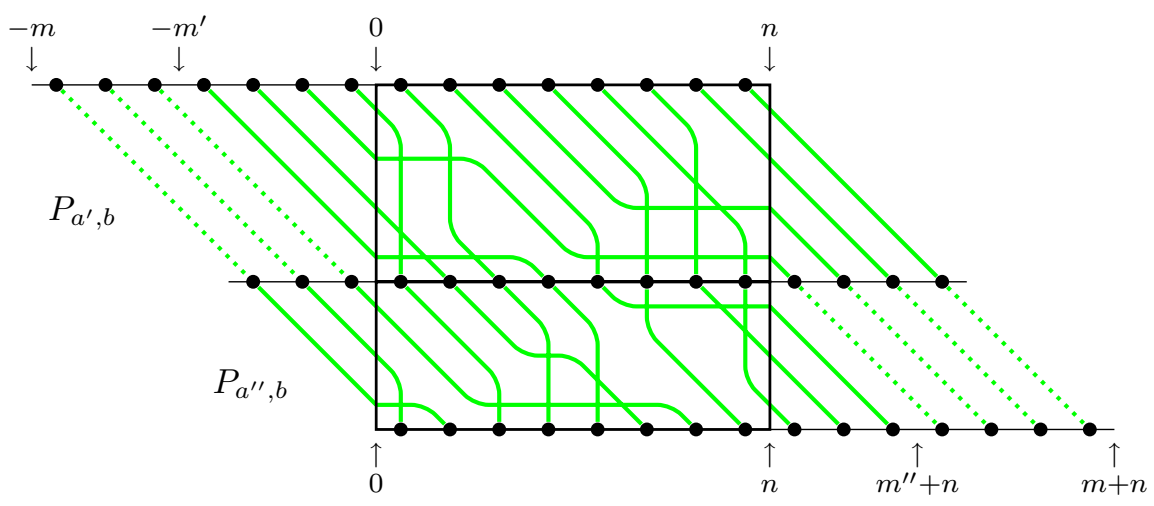

(a) Input matrices $P_{a^{\prime}, b}, P_{a^{\prime \prime}, b}$

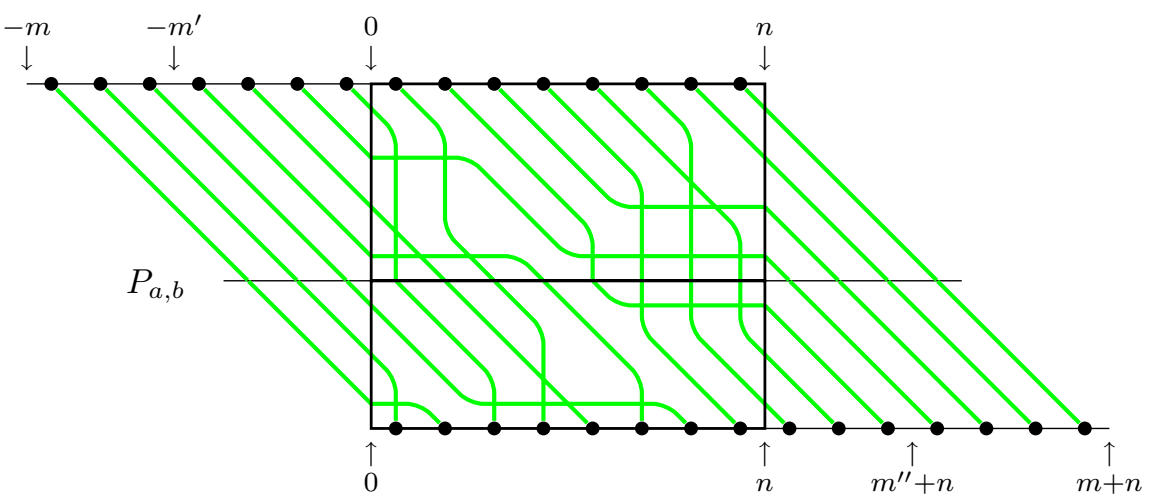

(b) Output matrix $P_{a, b}$

Figure 3.4: Semi-local score matrix composition: $P_{a^{\prime}, b} \boxminus P_{a^{\prime \prime}, b}=P_{a, b}$

Theorem 10 Given the nonzeros of the string-substring seaweed matrices $P_{a^{\prime}, b}^{s-s u b}, P_{a^{\prime \prime}, b}^{s-s u b}$, it is possible to compute the nonzeros of the string-substring seaweed matrix $P_{a, b}^{s-s u b}$ in time $O\left(n \log \min \left(m^{\prime}, m^{\prime \prime}, n\right)\right)$.

Proof We have

$$
\begin{aligned}
& H_{a, b}(i, k)=\max _{j \in[0: n]}\left(H_{a^{\prime}, b}(i, j)+H_{a^{\prime \prime}, b}(j, k)\right)= \\
& \max _{j \in[0: n]}\left(j-i-P_{a^{\prime}, b}^{\Sigma}(i, j)+k-j-P_{a^{\prime \prime}, b}^{\Sigma}(j, k)\right)= \\
& k-i-\min _{j \in[0: n]}\left(P_{a^{\prime}, b}^{\Sigma}(i, j)+P_{a^{\prime \prime}, b}^{\Sigma}(j, k)\right)= \\
& k-i-P_{a, b}^{\Sigma}(i, k)
\end{aligned}
$$

for all $i, k \in[0, n]$. Therefore, we have

$$
P_{a, b}^{\Sigma}(i, k)=\min _{j \in[0: n]}\left(P_{a^{\prime}, b}^{\Sigma}(i, j)+P_{a^{\prime \prime}, b}^{\Sigma}(j, k)\right)=\left(P_{a^{\prime}, b} \boxminus P_{a^{\prime \prime}, b}\right)(i, k)
$$


Hence, the string-substring seaweed submatrix for $a$ against $b$ can be obtained by implicit distance multiplication of the string-substring seaweed submatrices for $a^{\prime}$ against $b$ and for $a^{\prime \prime}$ against $b$ :

$$
P_{a, b}^{s-s u b}=P_{a^{\prime}, b}^{s-s u b} \boxminus P_{a^{\prime \prime}, b}^{s-s u b}
$$

If $n \leq \min \left(m^{\prime}, m^{\prime \prime}\right)$, then the theorem statement follows directly from Theorem 8. Otherwise, we may assume without loss of generality that $m^{\prime} \geq m^{\prime \prime}$, and that $\frac{n}{m^{\prime \prime}}$ is an integer. Since $P_{a^{\prime \prime}, b}$ is a superdiagonal matrix, by Lemma 3 we obtain a decomposition

$$
P_{a^{\prime \prime}, b}^{s-s u b}=P_{0} \boxminus \cdots \boxminus P_{\frac{n}{m^{\prime \prime}}-1} \boxminus I d_{m^{\prime \prime}}
$$

where each of $P_{0}, \ldots, P_{\frac{n}{m^{\prime \prime}}-1}$ is a permutation matrix embedding with core size $2 m^{\prime \prime}$. Then, we compute

$$
\begin{aligned}
& P_{a, b}^{s-s u b}= \\
& \quad P_{a^{\prime}, b}^{s-s u b} \boxminus P_{a^{\prime \prime}, b}\langle 0: n \mid 0: n\rangle= \\
& \quad P_{a^{\prime}, b}^{s-s u b} \boxminus\left(P_{0} \boxminus \cdots \boxminus P_{\frac{n}{m^{\prime \prime}}-1} \boxminus I d_{m^{\prime \prime}}\right)= \\
& \quad\left(\left(\left(P_{a^{\prime}, b}^{s-s u b} \boxminus P_{0}\right) \boxminus \cdots\right) \square P_{\frac{n}{m^{\prime \prime}}-1}\right) \square I d_{m^{\prime \prime}}
\end{aligned}
$$

By Lemma 2, each of the above implicit distance multiplications can be performed in-place in time $O\left(\mathrm{~m}^{\prime \prime} \log \mathrm{m}^{\prime \prime}\right)$. Therefore, all the nonzeros of matrix $P_{a, b}^{s-s u b}$ can be obtained in time $O\left(\frac{n}{m^{\prime \prime}} \cdot m^{\prime \prime} \log m^{\prime \prime}\right)=O\left(n \log m^{\prime \prime}\right)$.

Symmetrically, we have the following corollary.

Corollary 1 Given the nonzeros of the substring-string seaweed matrices $P_{a, b^{\prime}}^{s u b-s}, P_{a, b^{\prime \prime}}^{s u b-s}$, it is possible to compute the nonzeros of the substring-string seaweed matrix $P_{a, b}^{\text {sub-s }}$ in time $O\left(m \log \min \left(m, n^{\prime}, n^{\prime \prime}\right)\right)$.

Proof By Lemma 4 and Theorem 10.

Next, we extend this method to the three-way semi-local LCS problem.

Theorem 11 Given the nonzeros of the string-substring, prefix-suffix and suffix-prefix seaweed matrices

$$
\begin{array}{lll}
P_{a^{\prime}, b}^{s-s u b} & P_{a^{\prime}, b}^{p x-s x} & P_{a^{\prime}, b}^{s x-p x} \\
P_{a^{\prime \prime}, b}^{s-s u b} & P_{a^{\prime \prime}, b}^{p x-s x} & P_{a^{\prime \prime}, b}^{s x-p x}
\end{array}
$$

it is possible to compute the nonzeros of the string-substring, prefix-suffix and suffix-prefix seaweed matrices

$$
P_{a, b}^{s-s u b} \quad P_{a, b}^{p x-s x} \quad P_{a, b}^{s x-p x}
$$


as well as the nonzeros of the overlap submatrix of the substring-string seaweed matrix

$$
P_{a^{\prime}, a^{\prime \prime}, b}^{o s u b-s}
$$

in time $O\left(n \log \min \left(m^{\prime}, m^{\prime \prime}, n\right)\right)$.

Proof We first run the algorithm of Theorem 10, obtaining the nonzeros of $P_{a, b}^{s-s u b}$. We now need to obtain the nonzeros of $P_{a, b}^{p x-s x}, P_{a, b}^{s x-p x}, P_{a^{\prime}, a^{\prime \prime}, b}^{o s u b-s}$.

Matrix $P_{a, b}^{p x-s x}$ can be obtained by implicit distance multiplication of matrices $P_{a^{\prime}, b}^{s-s u b}$ and $P_{a^{\prime \prime}, b}^{p x-s x}$. The result of this multiplication has to be combined with matrix $P_{a^{\prime}, b}^{p x-s x}$, multiplied by an offset identity matrix:

$$
\begin{aligned}
& P_{a, b}^{p x-s x}=\left(P_{a^{\prime}, b}^{s-s u b} \boxminus P_{a^{\prime \prime}, b}^{p x-s x}\right) \sqcup \\
& \quad\left(P_{a^{\prime}, b}^{p x-s x} \boxminus I d_{m^{\prime \prime}}\left\langle n: m^{\prime}+n \mid m^{\prime \prime}+n: m+n\right\rangle\right)
\end{aligned}
$$

Symmetrically, matrix $P_{a, b}^{s x-p x}$ can be obtained by implicit distance multiplication of matrices $P_{a^{\prime}, b}^{s x-p x}$ and $P_{a^{\prime \prime}, b}^{s-s u b}$. The result of this multiplication has to be combined with matrix $P_{a^{\prime}, b}^{s x-p x}$, multiplied by an offset identity matrix:

$$
\begin{aligned}
& P_{a, b}^{s x-p x}=\left(P_{a^{\prime}, b}^{s x-p x} \square P_{a^{\prime \prime}, b}^{s-s u b}\right) \sqcup \\
& \quad\left(I d_{m^{\prime}}\left\langle-m:-m^{\prime} \mid-m^{\prime \prime}: 0\right\rangle \boxminus P_{a^{\prime \prime}, b}^{s x-p x}\right)
\end{aligned}
$$

Finally, matrix $P_{a^{\prime}, a^{\prime \prime}, b}^{o s u b-s}$ can be obtained by implicit distance multiplication of matrices $P_{a^{\prime}, b}^{s x-p x}$ and $P_{a^{\prime \prime}, b}^{p x-s x}$ :

$$
P_{a^{\prime}, a^{\prime \prime}, b}^{o s u b-s}=P_{a^{\prime}, b}^{s x-p x} \boxminus P_{a^{\prime \prime}, b}^{p x-s x}
$$

Similarly to Theorem 10, each of the above non-trivial implicit distance multiplications can be performed in time $O\left(n \log \min \left(m^{\prime}, m^{\prime \prime}, n\right)\right)$. The implicit distance multiplications by offset identity matrices can be performed in time $O(n)$. Therefore, the overall running time is $O\left(n \log \min \left(m^{\prime}, m^{\prime \prime}, n\right)\right)$.

Symmetrically, we have the following corollary.

Corollary 2 Given the nonzeros of the prefix-suffix, suffix-prefix and substringstring seaweed matrices

$$
\begin{array}{lll}
P_{a, b^{\prime}}^{p x-s x} & P_{a, b^{\prime}}^{s x-p x} & P_{a, b^{\prime}}^{s u b-s} \\
P_{a, b^{\prime \prime}}^{p x-s x} & P_{a, b^{\prime \prime}}^{s x-p x} & P_{a, b^{\prime \prime}}^{s u b-s}
\end{array}
$$

it is possible to compute the nonzeros of the prefix-suffix, suffix-prefix and substring-string seaweed matrices

$$
P_{a, b}^{p x-s x} \quad P_{a, b}^{s x-p x} \quad P_{a, b}^{s-s u b}
$$


as well as the nonzeros of the overlap submatrix of the string-substring seaweed matrix

$$
P_{a, b^{\prime}, b^{\prime \prime}}^{s-o s u b}
$$

in time $O\left(m \log \min \left(m, n^{\prime}, n^{\prime \prime}\right)\right)$.

Proof By Lemma 4 and Theorem 11.

While matrices $P_{a^{\prime}, a^{\prime \prime}, b}^{o s u b s}, P_{a, b^{\prime}, b^{\prime \prime}}^{s-s u b}$ do not contribute to the solution of the three-way semi-local LCS problem for $a$ against $b$, they are included in Theorem 11 and Corollary 2 for the sake of other applications, described in subsequent chapters.

Finally, we consider the full semi-local LCS problem.

Theorem 12 Given the nonzeros of the semi-local seaweed matrices $P_{a^{\prime}, b}$, $P_{a^{\prime \prime}, b}$, it is possible to compute the nonzeros of the semi-local seaweed matrix $P_{a, b}$ in time $O\left(m+n \log \min \left(m^{\prime}, m^{\prime \prime}, n\right)\right)$.

Proof We first run the algorithm of Theorem 11, obtaining the nonzeros of $P_{a, b}^{s-s u b}, P_{a, b}^{p x-s x}, P_{a, b}^{s x-p x}, P_{a^{\prime}, a^{\prime \prime}, b}^{o s u b-s}$. We now need to obtain the nonzeros of $P_{a, b}^{s u b-s}$. This is done by combining the overlap submatrix $P_{a^{\prime}, a^{\prime \prime}, b}^{o s u b}$ with matrices $P_{a^{\prime}, b}^{s u b-s}, P_{a^{\prime \prime}, b}^{s u b-s}$, each multiplied by an offset identity matrix:

$$
\begin{aligned}
& P_{a, b}^{s u b-s}=P_{a^{\prime}, a^{\prime \prime}, b}^{o s u b-s} \sqcup \\
& \quad\left(I d_{m^{\prime}}\left\langle-m:-m^{\prime} \mid-m^{\prime \prime}: 0\right\rangle \square P_{a^{\prime \prime}, b}^{s u b-s}\right) \sqcup \\
& \quad\left(P_{a^{\prime}, b}^{s u b-s} \boxminus I d_{m^{\prime \prime}}\left\langle n: m^{\prime}+n \mid m^{\prime \prime}+n: m+n\right\rangle\right)
\end{aligned}
$$

The algorithm of Theorem 11 runs in time $O\left(n \log \min \left(m^{\prime}, m^{\prime \prime}, n\right)\right)$. The implicit distance multiplications by offset identity matrices can each be performed in time $O(m)$. Therefore, the overall running time is $O(m+$ $\left.n \log \min \left(m^{\prime}, m^{\prime \prime}, n\right)\right)$.

Symmetrically, we have the following corollary.

Corollary 3 Given the nonzeros of the semi-local seaweed matrices $P_{a, b^{\prime}}$, $P_{a, b^{\prime \prime}}$, it is possible to compute the nonzeros of the semi-local seaweed matrix $P_{a, b}$ in time $O\left(m \log \min \left(m, n^{\prime}, n^{\prime \prime}\right)+n\right)$.

Proof By Lemma 4 and Theorem 12.

\subsection{Weighted scores and edit distances}

The concept of LCS score is generalised by that of (weighted) alignment score (see e.g. [61]). An alignment of strings $a, b$ is obtained by putting 
a subsequence of $a$ into one-to-one correspondence with a (not necessarily identical) subsequence of $b$, character by character and respecting the index order. The corresponding pair of characters, one from $a$ and the other from $b$, are said to be aligned. A character that is not aligned against a character of another string is said to be aligned against a gap in that string. Hence, four types of character alignment arise, each of which is given a real score:

- a pair of matching characters, with score $w_{=}$;

- a pair of mismatching characters, with score $w_{\#}$;

- a gap against a character, with score $w_{\perp}$;

- a character against a gap, with score $w_{\top}$.

Some of these scores may be negative. Aligning a matching pair of characters is considered to be better than aligning a mismatching pair of characters, which in its turn is not worse than aligning each of the two characters against a gap. Therefore, we assume $w_{=}>w_{\#} \geq w_{\perp}+w_{\top}$.

Example 13 The LCS score corresponds to taking

$$
w_{=}=1 \quad w_{\#}=w_{\perp}=w_{\top}=0
$$

A typical set of scores for DNA sequence alignment [28] is

$$
w_{=}=2 \quad w_{\#}=-1 \quad w_{\perp}=w_{\top}=-1.5
$$

Definition 20 The alignment score for strings $a, b$ is the maximum total score of character alignments in an alignment of $a$ and $b$.

We can extend naturally Definitions 12 and 13 to define the semi-local and the three-way semi-local alignment score problems. A semi-local alignment score corresponds to a highest-scoring path in a generalised alignment dag, where diagonal match, diagonal mismatch, horizontal and vertical edges have score $w_{=}, w_{\#}, w_{\perp}, w_{\top}$, respectively. The output of the semi-local alignment score problem corresponds to semi-local score matrix of a more general type than considered previously. This matrix is anti-Monge, but not necessarily unit-anti-Monge.

We show that without loss of generality, we can restrict ourselves to alignment scores with $w_{=}=1, w_{\perp}=w_{\top}=0$. Indeed, given arbitrary alignment scores, we transform them to normalised scores:

$$
w_{=}^{*}=1 \quad w_{\#}^{*}=\frac{w_{\#}-w_{\perp}-w_{\top}}{w_{=}-w_{\perp}-w_{\top}} \quad w_{\perp}^{*}=w_{\top}^{*}=0
$$


Then the score $w$ of any alignment with the original scores can be found from the score $w^{*}$ of the corresponding alignment with normalised scores as

$$
w=w^{*} \cdot\left(w_{=}-w_{\perp}-w_{\top}\right)+m \cdot w_{\top}+n \cdot w_{\perp}
$$

In this work, we will mostly restrict ourselves to alignment scores that satisfy the following rationality condition.

Definition 21 A set of character alignment scores will be called rational, if the corresponding normalised scores (in particular, $w_{\#}^{*}$ ) are all rational numbers.

Given a rational set of normalised scores, the semi-local alignment score problem on strings $a, b$ can be reduced to the semi-local LCS problem as follows. Let $w_{\#}^{*}=\frac{\mu}{\nu}<1$, where $\mu, \nu$ are positive natural numbers. From input strings $a, b$ of lengths $m, n$, we obtain strings $a^{\prime}, b^{\prime}$ of lengths $\nu m, \nu n$, by replacing every character $\gamma$ with a substring $\$^{\mu} \gamma^{\nu-\mu}$ of length $\nu$, where ' $\$$ ' is a special character not present in strings $a, b$. We have

$$
H_{a, b}(i, j)=\frac{1}{\nu} \cdot H_{a^{\prime}, b^{\prime}}(\nu i, \nu j)
$$

for all $i \in[-m: n], j \in[0: m+n]$, where the semi-local score matrix $H_{a, b}$ is defined by the original normalised scores, and the matrix $H_{a^{\prime}, b^{\prime}}$ by the LCS scores. Therefore, all the techniques of the previous sections apply to the more general semi-local alignment score problem, assuming that $\nu$ is a constant.

Finally, we can adapt the same approach to comparing strings by means of an edit distance metric. Here, we think of string $a$ being transformed into string $b$ by a sequence of weighted character edits of one of three types:

- character insertion, with weight $w_{i n}$;

- character deletion, with weight $w_{d e l}$;

- character substitution, with weight $w_{\text {sub }}$.

Definition 22 The edit distance between strings $a, b$ is the minimum total weight of a sequence of character edits transforming $a$ into $b$.

Example 14 The LCS distance (also called the indel distance) [90, 10], the Levenshtein distance (also called the indelsub distance) [78], and the episode distance [36] correspond respectively to

$$
\begin{aligned}
& w_{\text {in }}=w_{\text {del }}=1 \quad w_{\text {sub }}=2 \\
& w_{\text {in }}=w_{\text {del }}=w_{\text {sub }}=1 \\
& w_{\text {in }}=0 \quad w_{\text {del }} \geq w_{\text {sub }}>0
\end{aligned}
$$


The relationship between string alignment and string editing is straightforward: given a set of weights, the edit distance problem corresponds to the alignment score problem with scores

$$
w_{=}=0 \quad w_{\#}=-w_{\text {sub }} \quad w_{\perp}=-w_{\text {in }} \quad w_{\top}=-w_{\text {del }}
$$

This set of scores is rational, if $\frac{w_{d e l}+w_{i n}}{w_{s u b}}$ is a rational number. In such a case, all the techniques of the previous sections apply also to semi-local edit distances. 


\section{Chapter 4}

\section{The seaweed method}

\subsection{The seaweed algorithm}

A classical solution to the global LCS problem is given by the dynamic programming algorithm, discovered independently by Needleman and Wunsch (without an explicit analysis) [90], and by Wagner and Fischer [112]. This algorithm assumes a character comparison model that only allows comparison outcomes "equal" and "unequal", and the unit-cost RAM computation model. The dynamic programming algorithm runs in time $O(m n)$. In the course of the computation, the LCS problem is solved for all prefixes of input string $a$ against all prefixes of input string $b$.

A naive algorithm for the semi-local LCS problem runs in time $O((m+$ $n)^{4}$ ). Based on the ideas of Schmidt [101], Alves et al. [7] gave an algorithm for the string-substring LCS problem that runs in time $O(m n)$, which therefore extends the functionality of the standard dynamic programming algorithm, while matching its asymptotic running time. In the course of the computation, the string-substring LCS problem is solved for all prefixes of $a$ against all prefixes of $b$.

We now give a simple algorithm for the semi-local LCS problem, which further improves on the functionality of the above algorithms, while still matching their model assumptions and asymptotic running time. We call it the seaweed algorithm, since it has a simple interpretation in terms of seaweeds introduced in Chapter 1.

\section{Algorithm 1 (Semi-local LCS: The seaweed algorithm)}

Input: strings $a, b$ of length $m, n$, respectively.

Output: nonzeros of semi-local seaweed matrix $P_{a, b}$.

Description. We will maintain a variable permutation matrix $P$ over $\langle-m$ : $n|0: m+n\rangle$. We initialise it as an offset identity matrix:

$$
P \leftarrow I d_{m}\langle-m: n \mid 0: m+n\rangle
$$


The algorithm sweeps the alignment $\operatorname{dag} G_{a, b}$ in an arbitrary order compatible with the top-to-bottom and left-to-right partial order of the cells. For each cell, we perform an update on matrix $P$.

Consider a cell indexed by $\hat{l} \in\langle 0: m\rangle, \hat{\imath} \in\langle 0: n\rangle$. We define the cell's parameter characters to be the characters $a(\hat{l}), b(\hat{\imath})$. Let $i^{*}=\hat{\imath}+m-\hat{l}$. The update is performed on a $2 \times 2$ induced permutation submatrix of $P$ as follows:

$$
\begin{aligned}
& P\left\langle\cdot \mid i^{*}-1: i^{*}+1\right\rangle \leftarrow \\
& \begin{cases}\left(\begin{array}{ll}
0 & 1 \\
1 & 0
\end{array}\right) & \text { if } a(\hat{l}) \neq b(\hat{\imath}) \text { and } P\left\langle\cdot \mid i^{*}-1: i^{*}+1\right\rangle=\left(\begin{array}{ll}
1 & 0 \\
0 & 1
\end{array}\right) \\
\text { unchanged } & \text { otherwise }\end{cases}
\end{aligned}
$$

The sequence of updates on matrix $P$ can be interpreted as the following sequence of updates on the alignment dag. We start with a trivial fullmatch dag, which consists entirely of match cells. We then sweep the cells in the order described above. In each step, we transform a match cell into a mismatch cell, if its parameter characters mismatch. By Theorem 9, the algorithm maintains the invariant "current state of matrix $P$ is the semilocal seaweed matrix for the current state of the alignment dag". Therefore, at the end of the sweep, we have the output matrix

$$
P=P_{a, b}
$$

Cost analysis. For every cell, the $2 \times 2$ column-induced submatrix $P\left\langle\cdot, i^{*}-\right.$ $\left.1: i^{*}+1\right\rangle$ can be obtained from matrix $P$ in time $O(1)$. The cell update also runs in time $O(1)$. Therefore, the overall running time is $O(m n)$.

The memory cost is dominated by storing the input and the linear representation of the current matrix $P$. Therefore, the overall memory cost is $O(m+n)$.

In the course of the computation by Algorithm 1, the semi-local LCS problem is solved implicitly for all prefixes of $a$ against all prefixes of $b$. The algorithm can be interpreted in terms of seaweeds as follows. Each seaweed is traced across the alignment dag in the top-to-bottom or left-toright direction. A seaweed runs in a straight line by default; however, its direction may be affected by match cells, and by other seaweeds. Every cell has two seaweeds passing through it, one entering across the top edge and another across the left-hand edge. In a match cell, both seaweeds "bend away" from each other, so the seaweed entering at the top exits on the right, and the seaweed entering on the left exits at the bottom. In a mismatch cell, the two seaweeds keep straight and cross each other, if and only if this pair of seaweeds have not previously crossed; otherwise, they bend away as in a match cell. Therefore, any given pair of seaweeds are only allowed to cross at most once in the course of the computation. Notice that the same 


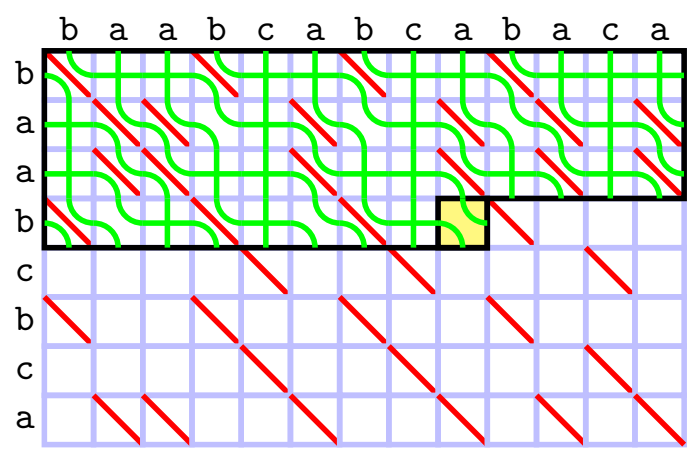

Figure 4.1: A snapshot of Algorithm 1 (the seaweed algorithm)

property of crossing at most once also holds for any pair of highest-scoring paths in the dag.

Example 15 Figure 4.1 shows a snapshot of Algorithm 1. The dag area that has already been processed is shown by the dark border; the cell currently being processed is shaded in yellow. Since the two seaweeds crossing in the current cell have previously crossed, the current step will leave the semilocal seaweed matrix unchanged, so that the second crossing is not allowed. The final layout of the seaweeds is identical to the one given in Figure 3.3, which describes the full sequence of states of the semi-local seaweed matrix in Algorithm 1.

Recall that the dag cells in Algorithm 1 can be processed in any order consistent with the left-to-right and top-to-bottom dependency partial order. In particular, the cell processing order can be fixed so that the algorithm will compute incrementally the semi-local seaweed matrix for all prefixes of string $a$ against string $b$. By keeping the algorithm's intermediate data, we obtain a data structure that allows efficient LCS queries for every prefix of $a$ against every substring of $b$. As in the classical dynamic programming approach, this data structure can be used to trace back (i.e. to obtain character by character) the actual LCS corresponding to a prefixsubstring LCS query, in time proportional to the size of the output (i.e. the length of the output subsequence). Alternatively, a technique similar to memory-efficient dynamic programming by Hirschberg [56] can be applied to achieve prefix-substring LCS traceback in the same asymptotic time, but in a linear amount of memory.

Under the "equal/unequal" character comparison model, Algorithm 1 matches the lower bound on the (global) LCS problem by Aho et al. [3] (see also a survey by Bergroth et al. [16] and references therein). Therefore, in this model the algorithm is asymptotically optimal. 


\subsection{Incremental LCS and semi-local LCS}

The incremental LCS problem was introduced by Landau et al. [75], and by Kim and Park [67]. Given a fixed text string, the problem asks its LCS score against a variable pattern string, which can be modified on-line by either appending or prepending a character. An extension, called fully-incremental LCS problem, was introduced by Ishida et al. [60]. Here, both input strings can be modified on-line in a similar fashion. In both versions of the problem, the goal is to maintain a data structure that will store the LCS score for the input strings, and will allow efficient on-line updates of this score.

Let $a, b$ denote the current state of each input string, and $m, n$ their respective current size. Works $[75,67]$ give incremental LCS algorithms with worst-case update time $O(m)$, where input string $a$ is kept fixed. Work [60] extends this result to a fully-incremental LCS algorithm with worst-case update time $O(m)$ (respectively, $O(n)$ ) when input string $a$ (respectively, $b$ ) is kept fixed.

We now give an algorithm for the fully-incremental LCS problem, matching the above algorithms in running time. Our algorithm is a straightforward generalisation of Algorithm 1. The dynamic data structure consists of the nonzeros of semi-local seaweed matrix $P_{a, b}$. When a new character is prepended or appended to string $a$ (respectively, $b$ ), the seaweed matrix is updated by processing a new row of cells along the top or bottom (respectively, column of cells along the left or right boundary) of the alignment dag $G_{a, b}$

The same technique also extends to the case where the dynamic data structure is required to support, in addition to the global LCS score, also semi-local LCS queries. By Theorem 1, a data structure allowing efficient semi-local LCS score queries can be maintained on top of the matrix $P_{a, b}$ at an extra time $O((m+n) \log (m+n))$ per character update.

We now give another generalisation of incremental string comparison. Consider a fixed text string of length $n$ and a variable pattern string, which can be modified on-line by either appending or prepending a block of characters from a pre-specified set of admissible blocks. The set of admissible blocks is known in advance, and off-line preprocessing of this set is allowed. The block-incremental LCS problem asks, as before, to maintain a data structure that will store the LCS score for the text against the pattern, and will allow efficient on-line updates of this score.

Consider an individual block update, and let $l$ be the corresponding block length. Such an update can be done naively as $l$ single-character updates, giving the block update time $O(n l)$.

We now give an algorithm for the block-incremental LCS problem, that improves on the naive algorithm in running time. The set of admissible blocks is preprocessed off-line by computing the string-substring seaweed matrix for the text string against every admissible block. The preprocessing 
runs in time $O(n \cdot L)$, where $L$ is the total length of the admissible blocks. Given the string-substring seaweed matrices, an individual block update can be performed by Theorem 10 in time $O(n \log l)$.

\subsection{Common-substring LCS and semi-local LCS}

The common-substring LCS problem was introduced by Landau et al. [77, 33]. Given a text string $b$ of length $n$ and an unspecified number of pattern strings, the problem asks for the LCS score of the text against each of the patterns. The pattern strings may share a known common substring $c$ of length $l$; we assume $l \leq n$. A pattern string may contain several copies of the common substring; the locations of all the copies are known in advance. The goal is, given the text, to preprocess the common substring so as to minimise the LCS computation time for each occurrence of the common substring in the patterns.

The problem can be solved naively by computing the LCS score for the text $b$ against each of the patterns, ignoring the common-substring structure. The resulting algorithm does no preprocessing, and runs in time $O(n l)$ for each occurrence of the common substring $c$.

An improved algorithm is given in [77,33]. This algorithm, following some preprocessing in time $O(n l)$, runs in time $O(n)$ for each occurrence of the common substring.

We now give an algorithm for the common-substring LCS problem, that matches the above algorithm both in preprocessing and running time. We preprocess the common substring $c$ in time $O(n l)$ by computing the nonzeros of the string-substring seaweed matrix $P_{c, b}^{s-s u b}$. For every pattern string $a$, we now compute the vector $h_{a, b}=H_{a, b}^{s-s u b}\langle 0 \mid *\rangle$ over $\langle 0: n\rangle$. This vector can be computed incrementally by repeated application of Theorem 6 . Each vector update takes time $O(n)$ per occurrence of the common substring $c$ in the pattern $a$, and the same time $O(n)$ per character of $a$ outside any occurrence of $c$.

We now consider the more general semi-local common-substring LCS problem. As in the ordinary semi-local LCS problem, string-substring, substringstring, prefix-suffix and suffix-prefix LCS score queries are now allowed between the text $b$ and each of the patterns.

The semi-local common-substring LCS problem can be solved naively by computing the implicit semi-local seaweed matrix for the text $b$ against each of the patterns, ignoring the common-substring structure. The resulting algorithm does no preprocessing, and runs in time $O(n l)$ for each occurrence of the common substring $c$.

We now give an algorithm for the semi-local common-substring LCS problem, improving on the naive algorithm in running time. We preprocess the common substring $c$ in time $O(n l)$ by computing the nonzeros of the 
semi-local seaweed matrix $P_{c, b}$. For every pattern string $a$, the semi-local seaweed matrix $P_{a, b}$ can now be computed incrementally, starting from an arbitrary occurrence of $c$ in $a$. The resulting algorithm can be regarded as a special case of the block-incremental LCS algorithm from Section 4.2. Each incremental update takes time $O(n \log l)$ per occurrence of the common substring $c$ in the pattern $a$, and $O(n)$ per character of $a$ outside any occurrence of $c$. Overall, the algorithm takes time $O(n)$ for the first occurrence of the common substring $c$ in a pattern, and time $O(n \log l)$ for each subsequent occurrence of $c$ in the same pattern. In particular, if the common substring occurs only once in every pattern string, our algorithm improves on the algorithm of $[77,33]$ in functionality, without any increase in the asymptotic running time. 


\section{Chapter 5}

\section{The micro-block seaweed method}

\subsection{The micro-block seaweed algorithm}

In the previous chapter, we assumed the character comparison model that only allows comparison outcomes "equal" and "unequal". As mentioned in Section 4.1, the LCS problem in this model has a lower bound of $\Omega(m n)$, which is matched by both the standard dynamic programming algorithm, and by the seaweed algorithm. Both these algorithms sweep the alignment dag cell by cell, and perform in every cell a constant amount of work, assuming the unit-cost RAM computation model.

We now switch to a more powerful character comparison model, assuming that the alphabet is a totally ordered set, and comparison outcomes are "less than", "equal" and "greater than". In this model, we no longer need to process every dag cell individually, so algorithms with running time $o(m n)$ become possible. This is true even if the computation model assumption is weakened, so that character comparisons and arithmetic operations are charged using the log-cost RAM model.

We assume without loss of generality that $m \leq n$, and that $m$ and $n$ are reasonably close, so $m=\Omega\left(\frac{\log n}{\log \log n}\right)$. In such a setting, LCS computation can be accelerated by the method originated by Arlazarov et al. [11], often called the "four Russians method". In this work, we call it the micro-block method, adapting the terminology of Bille and Gørtz [19]. The main idea of the method is to sweep the alignment dag in regular micro-blocks of a small, suitably chosen size, such that running time can be saved by precomputing all possible micro-block updates in advance. Using the micro-block method, Masek and Paterson [84] gave an algorithm for the (global) LCS problem running in time $O\left(\frac{m n}{\log ^{2} n}\right)$ for a constant-size alphabet ${ }^{1}$.

An extension of the subquadratic LCS algorithm to an unbounded-size

\footnotetext{
${ }^{1}$ The original algorithm by Masek and Paterson is described in [84] as running in time
} 
alphabet, running in time $O\left(\frac{m n(\log \log n)^{2}}{\log ^{2} n}\right)$ was suggested in [92], and fully developed by Bille and Farach-Colton [18]. In this extension, a second, coarser level of alignment dag partitioning is introduced. The blocks of this second level, called macro-blocks, are used for reducing the effective alphabet size, maximising the number of input string characters that fit into a machine word for each micro-block update.

We now give an algorithm for semi-local LCS running in subquadratic time, which presents a slight improvement on Algorithm 1. The algorithm uses the two-level micro-block method, similar to the global LCS algorithm of [18], and matching that algorithm in running time while providing a substantially more detailed string comparison. However, in contrast to the global LCS algorithms, the running time of our semi-local LCS algorithm cannot be reduced, even when the alphabet is of constant size.

\section{Algorithm 2 (Semi-local LCS: The micro-block seaweed algorithm)}

Input, output: as in Algorithm 1. Let $s=\frac{\log ^{2} n}{2}$ and $t=\frac{\log n}{8 \cdot \log \log n}$, where the logarithms are base 2 . We assume that $s \leq m \leq n$.

Description. Without loss of generality, we may assume that the alphabet size is at most $2 n$, and that the characters are encoded by values in the range $\langle-n: n\rangle$. We call two strings of equal length isomorphic, if one can obtained from the other by a permutation of the alphabet.

As in Algorithm 1, we will maintain a variable permutation matrix $P$ over $\langle-m: n \mid 0: m+n\rangle$. We initialise it as an offset identity matrix:

$$
P \leftarrow I d_{m}\langle-m: n \mid 0: m+n\rangle
$$

The algorithm sweeps the alignment $\operatorname{dag} G_{a, b}$ in square micro-blocks of size $t$. For each micro-block, we perform an update on matrix $P$.

Consider a micro-block indexed by $\langle l: l+t \mid i: i+t\rangle$, where $l \in[0: m-h]$, $i \in[0: n-h]$. We define the micro-block's parameter strings as the substrings $a\langle l: l+t\rangle, b\langle i: i+t\rangle$. Let $i^{*}=i+m-l$. The current micro-block can be regarded as a function, parameterised by the parameter strings, and performing an update on the $2 t \times 2 t$ column-induced permutation submatrix $P\left\langle\cdot \mid i^{*}-t: i^{*}+t\right\rangle$. The states of the submatrix before and after the updates will be called the micro-block's input submatrix and output submatrix, respectively. Note that both of these are permutation matrices, and therefore can be represented implicitly by their nonzeros.

The micro-blocks can be swept in an arbitrary order compatible with their top-to-bottom and left-to-right partial order. Combined with precomputation, this is already sufficient to obtain a subquadratic algorithm. However, in order to perform the computation even more efficiently, we introduce

$O\left(\frac{m n}{\log n}\right)$ for a constant-size alphabet in the log-cost RAM model. The unit-cost RAM version of the algorithm was described in $[115,18]$. 
a second level of macro-blocks of size $s$. We define a macro-block's parameter substrings, input and output submatrices similarly to a micro-block's ones.

The elements of a macro-block's parameter substrings are characters, encoded by values in the range $\langle-n: n\rangle$. The macro-block's input and output submatrices are represented by the row and column indices of the nonzeros; the natural range of these indices is also $\langle-n: n\rangle$. In order to perform the computation efficiently, we remap each of these ranges to a smaller range $\langle-s: s\rangle$ before passing the values to micro-blocks. The range remapping preserves the linear order of the values (both characters and matrix indices).

We process each macro-block as follows. First, we form its parameter substrings, and obtain the input submatrix from the preceding macro-blocks; overall, we have $O(s)$ characters and index values for the macro-block. Then, we sort the parameter substrings and the input submatrix indices in time $O(s \log s)$. We then remap these values from range $\langle-n: n\rangle$ to range $\langle-s: s\rangle$, preserving their relative order by using the sorting ranks. The remapping can be easily done in time $O(s)$. Then, we sweep all micro-blocks within the current macro-block, in an arbitrary order compatible with their topto-bottom and left-to-right partial order. For each micro-block, we form its parameter substrings, and obtain the input submatrix from the preceding macro-blocks; overall, we have $O(t)$ values for the micro-block of size $t$. For each micro-block, an update is then performed on a $2 t \times 2 t$ column-induced permutation submatrix of $P$ as follows:

$$
\begin{aligned}
& P\left\langle\cdot \mid i^{*}-t: i^{*}+t\right\rangle \\
& \quad \text { update }\left(a\langle l: l+t\rangle, b\langle i: i+t\rangle, P\left\langle\cdot \mid i^{*}-t: i^{*}+t\right\rangle\right)
\end{aligned}
$$

The parameter substrings and the input state consist each of $2 t$ values, ranging over $\langle-s: s\rangle$. For each of the at most $(2 s)^{2 t+2 t}=(2 s)^{4 t}$ possible combinations of the parameter and input values, the output values given by the function update are precomputed in advance, using Algorithm 1.

Similarly to Algorithm 1, the sequence of updates on matrix $P$ corresponds to the following sequence of updates on the alignment dag. We start with a trivial full-match dag. We then sweep the dag in micro-blocks of size $t$, in the order defined by the nested orders of macro- and micro-blocks. In each step, we transform a full-match micro-block into a micro-block defined by the input strings. The algorithm maintains the invariant "current state of matrix $P$ is the implicit semi-local score matrix for the current state of the alignment dag". Therefore, at the end of the sweep, we have the output matrix

$$
P=P_{a, b}
$$




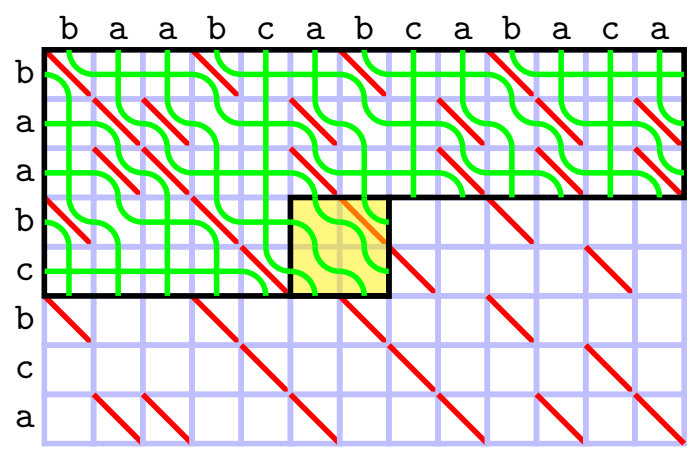

Figure 5.1: A snapshot of Algorithm 2 (the micro-block seaweed algorithm)

Cost analysis. In the precomputation stage, there are at most $(2 s)^{4 t}$ problem instances, each of which runs in time $O\left(t^{2}\right)$ in the log-cost RAM model. Therefore, the running time of the precomputation is

$$
\begin{aligned}
& O\left((2 s)^{4 t} \cdot t^{2}\right)=O\left(2^{\log \left(\log ^{2} n\right) \cdot \frac{\log n}{2 \log \log n}} \cdot t^{2}\right)= \\
& O\left(2^{2 \log \log n \cdot \frac{\log n}{2 \log \log n}} \cdot t^{2}\right)=O\left(2^{\log n} \cdot t^{2}\right)=o\left(n \log ^{2} n\right)
\end{aligned}
$$

which is negligible, compared to the subsequent main computation stage.

In the main computation stage, there are $\frac{m n}{t^{2}}$ micro-block update steps. The parameter substrings and input-output submatrices are each represented by $2 t$ values in the range $\langle-s: s\rangle$. The total micro-block data are of size

$$
O(2 t \cdot \log (2 s))=O\left(\frac{\log n}{\log \log n} \cdot \log \left(\log ^{2} n\right)\right)=O(\log n)
$$

and hence fit into a constant number of machine words. Therefore, the total running time of the algorithm is

$$
\frac{m n}{t^{2}} \cdot O(1)=O\left(\frac{m n(\log \log n)^{2}}{\log ^{2} n}\right)
$$

Example 16 Figure 5.1 shows a snapshot of Algorithm 2, using the same conventions as Figure 4.1. For simplicity, the macro-blocks are not shown, and the micro-blocks are assumed to be of size 2. As in Algorithm 1, the final layout of the seaweeds is identical to the one given in Figure 3.3.

\subsection{Cyclic LCS}

Given strings $a, b$ of length $m, n$ respectively, the cyclic LCS problem asks for the highest LCS score between $a$ and all cyclic shifts of $b$ (or, equivalently, all cyclic shifts of $a$ and all cyclic shifts of $b$ ). 
Cyclic string comparison has been considered by Maes [81], Bunke and Bühler [21], Landau et al. [75], Schmidt [101], Marzal and Barrachina [83]. Works $[75,101]$ give algorithms that solve the cyclic LCS problem in worstcase time $O(m n)$.

We now give a new algorithm for the cyclic LCS problem, improving on the existing algorithms in running time. First, we call Algorithm 2 on strings $a$ and $b b$ (a concatenation of string $b$ with itself), obtaining the semi-local seaweed matrix $P_{a, b b}$. Then, we perform $n$ string-substring LCS score queries for $a$ against every substring of $b b$ of length $n$; this can be done efficiently via Theorem 2. Finally, we take the maximum score among all the queries. The overall running time is dominated by the call to Algorithm 2, which runs in time $O\left(\frac{m n(\log \log n)^{2}}{\log ^{2} n}\right)$.

\subsection{Longest repeating subsequence}

Given a string $a$ of length $n$, the longest repeating subsequence problem asks for the length of the longest subsequence of $a$ that is a square, i.e. a concatenation of two identical strings.

This problem has been considered under the name "longest tandem scattered subsequence problem" by Kosowski [70], who gave an algorithm running in time $O\left(n^{2}\right)$.

We now give a new algorithm for the longest repeating subsequence problem, improving on the existing algorithm in running time. First, we call Algorithm 2 on string a against itself, obtaining the semi-local seaweed matrix $P_{a, a}$. Then, we perform $n-1$ prefix-suffix LCS score queries for every possible non-trivial prefix-suffix decomposition of $a$; this can be done efficiently via Theorem 2 . Finally, we take the maximum score among all the queries. The overall running time is dominated by the call to Algorithm 2, which runs in time $O\left(\frac{n^{2}(\log \log n)^{2}}{\log ^{2} n}\right)$.

\subsection{Approximate pattern matching}

Approximate pattern matching is a classical generalisation of both the alignment score (or, equivalently, edit distance) problem, and of ordinary pattern matching. Given a text string $t$ of length $n$ and a pattern string $p$ of length $m$, the approximate pattern matching problem asks to find the substrings of the text that are locally closest to the pattern, i.e. that have the locally highest alignment score (or, equivalently, lowest edit distance) against the pattern. The precise definition of "locally" may vary in different versions of the problem. Some well-known types of approximate pattern matching are as follows.

The local subsequence recognition problem, also called the minimal- 
window subsequence recognition problem, asks for all inclusion-minimal substrings in the text containing the pattern as a substring. Assuming the LCS alignment score, this corresponds to all $\lessgtr$-minimal points in the set

$$
\mathcal{H}_{m}=\left\{(i, j) \in[0: n \mid 0: n], \text { such that } H_{p, t}^{s-s u b}(i, j)=m\right\}
$$

If the above set is nonempty (i.e. if $p$ is contained in $t$ as a subsequence), it is the set of all global maxima in the matrix $H_{p, t}^{s-s u b}$. Another variant of this problem, the fixed-window subsequence recognition problem, given a window length $w$, asks for all substrings of length $w$ of the text containing the pattern as a substring. This corresponds to all points lying on the intersection of the diagonal $j-i=w$ with the set $\mathcal{H}_{m}$.

The threshold approximate matching problem assumes an alignment score with arbitrary weights, and, given a threshold score $h$, asks for all substrings of the text that have alignments score at least $h$ against the pattern. This corresponds to all points in the set

$$
\mathcal{H}_{h}=\left\{(i, j) \in[0: n \mid 0: n], \text { such that } H_{p, t}^{s-s u b}(i, j) \geq h\right\}
$$

The complete approximate matching problem assumes an alignment score with arbitrary weights, and for every suffix of the text, asks for a prefix of this suffix that has the highest alignment score against the pattern. This corresponds to the set of all row maxima of the stringsubstring score matrix $H_{p, t}^{s-s u b}$.

The local subsequence recognition problem is considered by Das et al. [36] as the "episode matching problem". For both the minimal-window and fixed-window versions, they give an algorithm running in time $O\left(\frac{m n}{\log ^{2} n}\right)$ for a constant-size alphabet, which can be modified to an algorithm running in time $O\left(\frac{m n(\log \log n)^{2}}{\log ^{2} n}\right)$ for an unbounded-size alphabet. A multi-pattern version of the problems has been considered by Cégielski et al. [25].

The complete (and therefore also the threshold) approximate pattern matching problem is solved by a classical algorithm by Sellers [102] in time $O(m n)$. Assuming a rational set of weights, the micro-block method gives an algorithm running in time $O\left(\frac{m n}{\log ^{2} n}\right)$ for a constant-size alphabet, and in time $O\left(\frac{m n(\log \log n)^{2}}{\log ^{2} n}\right)$ for an unbounded-size alphabet. Various extensions of the problem have been considered by Landau and Vishkin [76], Cormode and Muthukrishnan [32] and many others (see e.g. a survey by Navarro [89] and references therein).

The threshold approximate pattern matching problem also admits algorithms with running time parameterised by the threshold score $h$, or the corresponding threshold edit distance. When the threshold edit distance is 
low, the best known algorithm is by Cole and Hariharan [31]. We do not explore parameterised approximate pattern matching any further in this work.

We now give a new unified algorithm for all the described versions of approximate pattern matching. Our algorithm matches the algorithms based on the micro-block method in running time for an unbounded-size alphabet.

The new algorithm is as follows. First, we call Algorithm 2 on strings $p, t$ (in case of rational weights, via the subdivided alignment dag of Section 3.4), obtaining the semi-local seaweed matrix $P_{p, t}$. By Theorem 1, we then build a data structure that allows to query any element of the semi-local score matrix $H_{p, t}$ in polylogarithmic time. Since matrix $H_{p, t}$ is anti-Monge, all the row maxima can now be found efficiently by the algorithm of Lemma 1, which solves the complete approximate matching problem. The threshold approximate matching problem can now be solved by considering the matrix entries in the neighbourhood of the row maxima. Both the minimal-window and the fixed-window versions of the local subsequence recognition problem can be solved by selecting the row maxima that satisfy the additional conditions imposed by the problem.

In all the described versions of the algorithm, the overall running time is dominated by the call to Algorithm 2, which runs in time $O\left(\frac{m n(\log \log n)^{2}}{\log ^{2} n}\right)$. 


\section{Chapter 6}

\section{Periodic string comparison}

\subsection{The periodic seaweed algorithm}

In many string comparison applications, one or both of the input strings may have periodic structure. In this chapter, we show how to exploit such structure efficiently, using a variant of the seaweed method.

Consider the problem of comparing a finite string $a$ of length $m$ against a string $b$, which is infinite in both directions and periodic: $b=u^{ \pm \infty}=$ ...uиuu... The period string $u$ is finite of length $p$.

Definition 23 Given strings $a$, $u$, the periodic string-substring LCS problem asks for the LCS score of $a$ against every finite substring of $b=u^{ \pm \infty}$.

Without loss of generality, we assume that every character of $a$ occurs in $u$ at least once. Clearly, the length of the substring of $b$ in Definition 23 can be restricted to be at most $m p$ (otherwise, every character of $a$ can be matched to a different copy of $u$ within $b$, and therefore the string-substring LCS score is equal to $m$ ).

The definition of the alignment dag (Definition 14) extends naturally to the periodic string-substring LCS problem. The alignment dag is itself periodic: the edges $v_{l, \hat{\imath}-\frac{1}{2}+k p} \rightarrow v_{l, \hat{\imath}+\frac{1}{2}+k p}$ (respectively, $v_{\hat{l}-\frac{1}{2}, i+k p} \rightarrow v_{\hat{l}+\frac{1}{2}, i+k p}$, $\left.v_{\hat{l}-\frac{1}{2}, \hat{\imath}-\frac{1}{2}+k p} \rightarrow v_{\hat{l}+\frac{1}{2}, \hat{\imath}+\frac{1}{2}+k p}\right)$ have equal scores for all $l \in\left[l_{0}: l_{1}\right], \hat{l} \in\left\langle l_{0}: l_{1}\right\rangle$, $i \in\left[i_{0}, i_{1}\right], \hat{\imath} \in\left\langle i_{0}: i_{1}\right\rangle, k \in[-\infty:+\infty]$. Such an alignment dag can also be regarded as a horizontal composition of an infinite sequence of period subdags, each of which is isomorphic to the $m \times p$ alignment dag $G_{a, u}$.

Since string $b$ is infinite, the semi-local score and seaweed matrices coincide with the respective string-substring submatrices: matrix $H_{a, b}=H_{a, b}^{s-s u b}$ is over $[-\infty:+\infty]$, and matrix $P_{a, b}=P_{a, b}^{s-s u b}$ is over $\langle-\infty:+\infty\rangle$. Furthermore, matrices $H_{a, b}, P_{a, b}$ are again periodic: we have

$$
\begin{aligned}
& H_{a, b}(i, j)=H_{a, b}(i+p, j+p) \\
& P_{a, b}(\hat{\imath}, \hat{\jmath})=P_{a, b}(\hat{\imath}+p, \hat{\jmath}+p)
\end{aligned}
$$


for all $i, j \in[-\infty: \infty], \hat{\imath}, \hat{\jmath} \in\langle-\infty: \infty\rangle$. To represent such matrices, it is sufficient to store the $p$ nonzeros of the horizontal period submatrix $P_{a, b}\langle 0$ : $p|*\rangle$, or, symmetrically, of the vertical period submatrix $P_{a, b}\langle * \mid 0: p\rangle$. The nonzero sets of the two period submatrices can be obtained from one another in time $O(p)$; we will be using both of them simultaneously where necessary.

The periodic string-substring LCS problem can be solved by a simple extension of the seaweed algorithm (Algorithm 1). Since the semi-local seaweed matrix is periodic, the seaweeds only need to be traced within a single period subdag, with appropriate wraparound.

\section{Algorithm 3 (Periodic string-substring LCS: The periodic seaweed algorithm)}

Input: strings $a, u$ of length $m, p$, respectively.

Output: nonzeros of (say) vertical period submatrix $P_{a, b}\langle * \mid 0: p\rangle$, where $b=u^{ \pm \infty}$.

Description. We will maintain a variable permutation matrix $P$ over $\langle-\infty$ : $+\infty\rangle$, with period $p$ and vertical period submatrix $P\langle * \mid 0: p\rangle$. We initialise it as an offset identity matrix (which is a periodic matrix for any period):

$$
P\langle * \mid 0: p\rangle \leftarrow I d_{m}\langle * \mid 0: p\rangle
$$

Then, we sweep the cells of the period subdag as follows. In the outer loop, we run through the rows of cells top-to-bottom. For the current row $\hat{l} \in\langle 0: m\rangle$, we start the inner loop at an arbitrary match cell $\hat{\imath}_{0} \in\langle 0: p\rangle$, so we have $a(\hat{l})=b(\hat{\imath})$. Such a match cell is guaranteed to exist by the assumption that every character of $a$ occurs in $u$ at least once. Then, we sweep the cells from $\hat{\imath}=\hat{\imath}_{0}$ left-to-right, wrapping around from $\hat{\imath}=p-\frac{1}{2}$ to $\hat{\imath}=\frac{1}{2}$, and continuing the sweep left-to-right up to $\hat{\imath}=\hat{\imath}_{0}-1$. For each cell, we perform an update on matrix $P$. At the end of the sweep, we will have $P=P_{a, b}$.

Consider a cell indexed by $\hat{l} \in\langle 0: m\rangle, \hat{\imath} \in\langle 0: p\rangle$. We define the cell's parameter characters to be $a(\hat{l}), b(\hat{\imath})$. Let $i^{*}=\hat{\imath}+m-l$. As in Algorithm 1 , the update is performed on a $2 \times 2$ column-induced permutation submatrix of $P$ as follows:

$$
\begin{aligned}
& P\left\langle\cdot \mid i^{*}-1: i^{*}+1\right\rangle \leftarrow \\
& \begin{cases}\left(\begin{array}{ll}
0 & 1 \\
1 & 0
\end{array}\right) & \text { if } a(\hat{l}) \neq b(\hat{\imath}) \text { and } P\left\langle\cdot \mid i^{*}-1: i^{*}+1\right\rangle=\left(\begin{array}{ll}
1 & 0 \\
0 & 1
\end{array}\right) \\
\text { unchanged } & \text { otherwise }\end{cases}
\end{aligned}
$$

Note that the first update in an inner loop is always trivial: we have $a(\hat{l})=$ $b\left(\hat{\imath}_{0}\right)$, therefore $P$ remains unchanged.

The sequence of updates on matrix $P$ can be interpreted as a sequence of updates on the alignment dag, as described in Algorithm 1, but now 


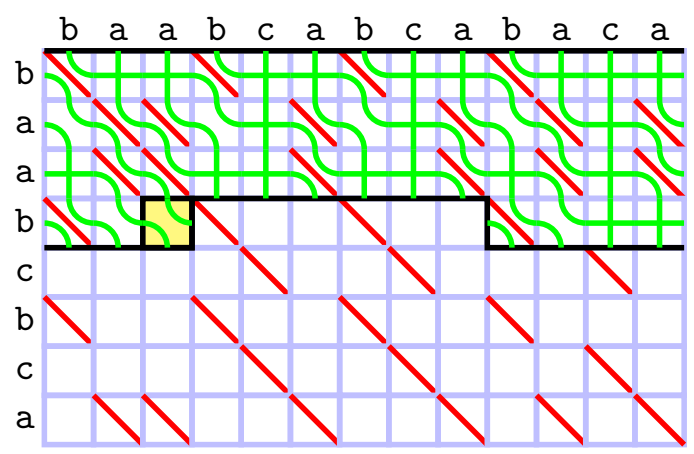

Figure 6.1: A snapshot of Algorithm 3 (the periodic seaweed algorithm)

including the wraparound. Therefore, at the end of the sweep, we have the output period submatrix

$$
P\langle * \mid 0: p\rangle=P_{a, b}\langle * \mid 0: p\rangle
$$

Cost analysis. For every cell, the $2 \times 2$ column-induced submatrix $P\langle\cdot|$ $\left.i^{*}-1: i^{*}+1\right\rangle$ can be obtained from the vertical period submatrix of $P$ in time $O(1)$. The cell update also runs in time $O(1)$. Therefore, the overall running time is $O(m p)$.

The memory cost is dominated by storing the input and the period submatrix of the current matrix $P$. Therefore, the overall memory cost is $O(m+p)$.

Example 17 Figure 6.1 shows a snapshot of Algorithm 3, using the same conventions as Figure 4.1.

Note that the cell updating order in Algorithm 3 is significantly more restricted than in Algorithm 1, due to the extra data dependencies caused by the wraparound. This seems to rule out the possibility of a micro-block version of the algorithm.

\subsection{Tandem alignment}

The periodic LCS problem has many variations that can be solved by an application of the periodic seaweed algorithm.

The first such variation is the tandem LCS problem. The problem asks for the LCS score of a string $a$ of length $m$ against a tandem $k$-repeat string $b=u^{k}$ of length $n=k p$. As before, we assume that every character of $a$ occurs in $u$ at least once; we may also assume that $k \leq m$.

The tandem LCS problem can be solved naively by considering the LCS problem directly on strings $a$ and $b$, ignoring the periodic structure of string 
b. The standard dynamic programming algorithm (see Section 4.1) solves the problem in time $O(m n)=O(m k p)$. This running time can be slightly improved by the micro-block method (see Section 5.1).

The tandem LCS problem can also be regarded as a special case of the common-substring LCS problem [77, 33] (see also Section 4.3). Using this technique, the problem can be solved in time $O(m(k+p))$. The techniques of Landau et al. [33, 74] give an algorithm for the tandem LCS problem, parameterised by the LCS score of the input strings; however, the worst-case running time of this algorithm is still $O(m(k+p))$. Landau [73] asked if the running time for the tandem LCS problem can be improved to $O(m(\log k+$ $p))$.

We now give an algorithm that improves on the current algorithms in time and functionality, and even exceeds Landau's expectation. First, we call Algorithm 3 on strings $a$ and $u$. Then, we count the number of nonzeros $\lessgtr$-dominated by point $(0, n)$, i.e. nonzeros in the submatrix $P_{a, b}\langle 0:+\infty|$ $-\infty: n\rangle$. Given the (say) horizontal period submatrix $P_{a, b}\langle 0: p \mid *\rangle$, this can be done by a sweep of its $p$ nonzeros, counting every nonzero with appropriate multiplicity. More precisely, every nonzero $P_{a, b}(i, j)=1, i \in$ $\langle 0: p\rangle, j \in\langle-\infty: \infty\rangle$, is counted with multiplicity $k-\lfloor j / p\rfloor$, if $j \in\langle 0: n\rangle$, and is skipped (counted with multiplicity 0 ) otherwise. The solution to the tandem LCS problem is then obtained by Theorem 9. The overall running time is dominated by the call to Algorithm 3, which runs in time $O(m p)$.

Another set of variations on the periodic LCS problem was introduced by Benson [14] as the tandem alignment problem. Instead of asking for all string-substring LCS scores of $a$ against $b=u^{ \pm \infty}$, the tandem alignment problem asks for a substring of $b$ that is closest to $a$ in terms of alignment score (or edit distance), under different restrictions on the substring. In particular:

- the pattern global, text global (PGTG) tandem alignment problem restricts the substring of $b$ to consist of a whole number of copies of $u$, i.e. to be of the form $u^{k}=u u \ldots u$ for an arbitrary integer $k$;

- the tandem cyclic alignment problem restricts the substring of $b$ to be of length $k p$ for an arbitrary integer $k$ (but it may not consist of a whole number of copies of $u$ );

- the pattern local, text global (PLTG) tandem alignment problem leaves the substring of $b$ unrestricted.

All three versions of the tandem alignment problem can be regarded as special cases of the approximate pattern matching problem (see Section 5.4) on strings $a$ of length $m$ and $b^{\prime}=u^{m}$ of length $n=m p$ (but with the roles of the text and the pattern reversed). Therefore, the tandem LCS problem can be solved naively by considering the approximate pattern matching problem 
directly on strings $a$ and $b^{\prime}$, ignoring the periodic structure of string $b^{\prime}$. Given an arbitrary (real) set of alignment weights, the classical algorithm by Sellers (see Section 5.4) solves the problem in time $O(m n)=O\left(m^{2} p\right)$. For a rational set of weights, the running time can again be slightly improved by the micro-block precomputation method (see Section 5.4).

The PGTG and PLTG tandem alignment problems can be solved more efficiently by the technique of wraparound dynamic programming [87, 45] (see also [14]) in time $O(m p)$. For the tandem cyclic alignment problem, Benson [14] modified this technique to give an algorithm running in time $O(m p \log p)$ and memory $O(m p)$.

We now give a new algorithm for the tandem cyclic alignment problem, which improves on the existing algorithm in running time, assuming a rational set of alignment weights. The running time of the new algorithm matches the current algorithms for the PGTG and PLTG tandem alignment problems.

Given input strings $a, u$, we first solve the periodic string-substring problem by calling Algorithm 3. This gives us a period submatrix of matrix $P_{a, b}$, where $b=u^{ \pm \infty}$. Then, for each $k, 0<k<m$, we perform independently the following procedure. We solve the tandem LCS problem for strings $a$ and $u^{k}$ by the method described earlier in this section, counting every nonzero in the period submatrix $P_{a, b}$ with an appropriate multiplicity. This gives us the LCS score for $a$ against $u^{k}$ for every $k$. We then update this score incrementally, obtaining the LCS score for string $a$ against a window of length $p$ in $b$, sliding through $p$ successive positions. This is equivalent to querying $p$ successive elements in a diagonal of matrix $P_{a, b}$, which can be achieved by $2 p$ incremental dominance counting queries. By Theorem 2, every one of these queries can be performed in time $O(1)$.

The call to Algorithm 3 runs in time $O(m p)$; its output is shared by the tandem LCS computation for all $k$. For each $k$, the running time of the remaining computation is $O(p)$. Therefore, the combined running time for all values of $k$ is $m \cdot O(p)=O(m p)$. Overall, the algorithm runs in time $O(m p)$. 


\section{Chapter 7}

\section{Permutation string comparison}

\subsection{Semi-local LCS between permutations}

An important special case of string comparison is where each of the input strings $a, b$ is a permutation string, i.e. a string that consists of all distinct characters. Without loss of generality, we may assume that $m=n$, and that both strings are permutations of a given totally ordered set of size $n$. The semi-local LCS problem on permutation strings is equivalent to the following classical problem.

Definition 24 Given a string a, the longest increasing subsequence (LIS) problem asks for the length of the longest string that is an increasing subsequence of $a$.

The LIS problem has a long history, going back to Erdös and Szekeres [42] and Robinson [97]. Later, Knuth [69], Fredman [46] and Dijkstra [40] gave algorithms running in time $O(n \log n)$. The problem was studied further by Chang and Wang [27] and by Bespamyatnikh and Segal [17].

The semi-local LCS problem on permutation strings is equivalent to solving the LIS problem in every substring of a given string. In the rest of this section, we give an efficient algorithm for this problem.

For consistency with the notation in previous chapters, we will assume that a permutation string of length $n$ is indexed by odd half-integers $\langle 0: n\rangle$, and is over the alphabet $\langle 0: n\rangle$, unless indicated otherwise. The identity permutation string of length $n$ is the string $i d=\left(\frac{1}{2}, \frac{3}{2}, \ldots, n-\frac{1}{2}\right)$.

Given a string $a$, we denote its reverse string by $\bar{a}$. In particular, the reverse identity permutation string is $\overline{i d}=\left(n-\frac{1}{2}, n-\frac{3}{2}, \ldots, \frac{1}{2}\right)$. We denote by $\Sigma(a)$ the set of characters appearing in $a$ at least once. For a set of characters $S$, we denote by $\left.a\right|_{S}$ the filtered subsequence of $a$, which consists only of those characters that belong to $S$. 


\section{Algorithm 4 (Semi-local LCS between permutation strings)}

Input: permutation strings $a, b$ of length $n$ over an alphabet of size $n$.

Output: nonzeros of the semi-local seaweed matrix $P_{a, b}$.

Description. Recursion on $n$.

Recursion base. If $n=1$, the computation is trivial.

Recursive step. Assume without loss of generality that $n>1$ is even. We partition the input string $a$ into a concatenation $a=a^{\prime} a^{\prime \prime}$ of two strings of length $\frac{n}{2}$. Each of the strings $a^{\prime}, a^{\prime \prime}$ is a permutation string of length $\frac{n}{2}$.

The semi-local seaweed matrices $P_{a^{\prime}, b}, P_{a^{\prime \prime}, b}$, are each over $\left\langle-\frac{n}{2}: n\right| 0$ : $\left.\frac{3 n}{2}\right\rangle$, and each contain $\frac{3 n}{2}$ nonzeros. The semi-local seaweed matrix $P_{a, b}$ is over $\langle-n: n \mid 0: 2 n\rangle$, and contains $2 n$ nonzeros.

Note that for all $\hat{\imath} \in\langle 0: n\rangle$, we have $P_{a^{\prime}, b}(\hat{\imath}, \hat{\imath})=1$, whenever $b(\hat{\imath}) \notin \Sigma\left(a^{\prime}\right)$. More formally, let $\langle 0: n\rangle=I^{\prime} \cup I^{\prime \prime}$, where

$$
\begin{aligned}
& I^{\prime}=\left\{\hat{\imath} \in\langle 0: n\rangle, \text { such that } b(\hat{\imath}) \in \Sigma\left(a^{\prime}\right)\right\} \\
& I^{\prime \prime}=\left\{\hat{\imath} \in\langle 0: n\rangle, \text { such that } b(\hat{\imath}) \in \Sigma\left(a^{\prime}\right)\right\}
\end{aligned}
$$

Sets $I^{\prime}, I^{\prime \prime}$ can be computed easily from strings $a^{\prime}, a^{\prime \prime}, b$, at the cost of sorting their character sets. We have a decomposition

$$
P_{a^{\prime}, b}=P_{a^{\prime}, b}\left(\left\langle-\frac{n}{2}: 0\right\rangle \cup I^{\prime} \mid I^{\prime} \cup\left\langle n: \frac{3 n}{2}\right\rangle\right) \sqcup I d\left(I^{\prime \prime} \mid I^{\prime \prime}\right)
$$

The two permutation matrices in the above decomposition are of size $n$ and $\frac{n}{2}$, respectively. Only the $n$ nonzeros in the former matrix are non-trivial; they can be obtained by solving recursively the semi-local LCS problem on strings $a^{\prime}$ and $\left.b\right|_{\Sigma\left(a^{\prime}\right)}=b\left(I^{\prime}\right)$, both of which are permutations strings with alphabet size $\frac{n}{2}$. Similarly, only $n$ out of $\frac{3 n}{2}$ nonzeros of $P_{a^{\prime \prime}, b}$ are non-trivial; they can be obtained by solving recursively the semi-local LCS problem on strings $a^{\prime \prime}$ and $\left.b\right|_{\Sigma\left(a^{\prime \prime}\right)}=b\left(I^{\prime \prime}\right)$.

Finally, given matrices $P_{a^{\prime}, b}, P_{a^{\prime \prime}, b}$, the output matrix $P_{a, b}$ is computed by a call to the algorithm of Theorem 12, which calls the algorithm of Theorem 8 as a subroutine. Note that we now have two nested recursions: the current recursion, and the recursion of Theorem 8 .

End of recursive step.

Cost analysis. The recursion tree is a balanced binary tree of height $\log n$. In the root node, the running time is dominated by the call to the algorithm of Theorem 12, and is therefore $O(n \log n)$. In each subsequent level of the recursion tree, the number of nodes doubles, and the running time per node is reduced by at least a factor of 2 . Therefore, the running time per level is $O(n \log n)$. The overall running time is $\log n \cdot O(n \log n)=O\left(n \log ^{2} n\right)$. 

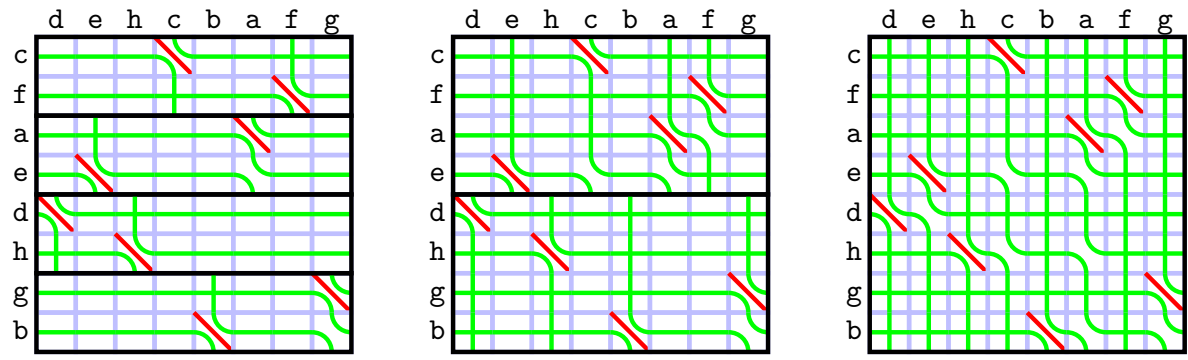

Figure 7.1: Snapshots of Algorithm 4 (semi-local LCS between permutation strings)

Example 18 Figure 7.1 shows a series of snapshots of an execution of Algorithm 4 on permutation strings $a=$ "cfaedhgb", $b=$ "dehcbafg".

By keeping the algorithm's intermediate data, we obtain a data structure that allows efficient traceback of any semi-local LCS query on a pair of permutations, in time proportional to the size of the output (i.e. the length of the output subsequence). A related problem of tracing back LIS in every substring of a fixed size in a permutation has been studied by Albert et al. [6] and by Chen et al. [29]. In particular, work [29] gives an algorithm that runs in time proportional to the size of the output (i.e. the combined lengths of all the output subsequences). In the same work, the algorithm is also generalised for tracing back the LIS in an arbitrary subset of $n$ substrings, possibly of different sizes. In both versions of the problem, the size of the output, and therefore the algorithm's running time, can be as high as $\Theta\left(n^{2}\right)$. In contrast, Algorithm 4 allows efficient LIS traceback for any individual substring.

\subsection{Cyclic LCS between permutations}

The cyclic LCS problem has been defined in Section 5.2. Given permutation strings $a, b$ of length $n$, the cyclic LCS problem on $a, b$ is equivalent to the LIS problem on a circular string.

This problem has been considered by Albert et al. [5], who gave a Monte Carlo randomised algorithm, running in time $O\left(n^{1.5} \log n\right)$ with small error probability.

We now give a new algorithm for cyclic LCS between permutations, that improves on the above algorithm both in running time, and by being deterministic. First, we call Algorithm 4, obtaining the semi-local seaweed matrix $P_{a, b}$. Then, we run the algorithm of Theorem 12 on matrix $P_{a, b}$ against itself, obtaining the semi-local seaweed matrix $P_{a a, b}$. Finally, we perform $n$ substring-string LCS queries for every substring of $a a$ of length 
$n$ against string $b$. The overall running time is dominated by the call to Algorithm 4, which runs in time $O\left(n \log ^{2} n\right)$.

A version of the cyclic LCS problem between permutations, parameterised by the output LCS length $l$, has also been considered by Albert et al. [5], who gave an algorithm running in time $O(n l \log n)$. This was improved upon by Deorowicz [39], who gave an algorithm running in time $O\left(\min \left(n l, n \log n+l^{3} \log n\right)\right)$. Our algorithm described above improves on the algorithm of [39], unless $l=o\left((n \log n)^{1 / 3}\right)$.

\subsection{Longest pattern-avoiding subsequences}

Two given permutation strings $a, b$ of equal length (but generally over different alphabets) are called isomorphic, if they have the same relative order of characters, i.e. $a(\hat{\imath})<a(\hat{\jmath})$ iff $b(\hat{\imath})<b(\hat{\jmath})$ for all $\hat{\imath}, \hat{\jmath}$. Given a target permutation string $t$ of length $n$ and a pattern permutation string $p$ of fixed length, the longest p-isomorphic subsequence problem, or simply the longest $p$-subsequence problem, asks for the longest subsequence of $t$ that is isomorphic to $p$. More generally, given a set of pattern permutation strings $X$, the longest $X$-subsequence problem asks for the longest subsequence of $t$ that is isomorphic to any pattern string in $p$.

Example 19 The LIS problem can be interpreted as the longest $X$-subsequence problem, where $X$ is a set of identity permutation strings, one of each length $m \in[1: n]$.

Given a set of antipattern permutation strings $Y$, the longest $Y$-avoiding subsequence problem asks for the longest subsequence of $t$ that does not contain a subsequence isomorphic to any string in $Y$.

Example 20 The LIS problem on a permutation string can be interpreted as the longest $\{$ "21" $\}$-avoiding subsequence problem.

For a detailed introduction into these problems and their connections, see the work by Albert et al. [4] and references therein.

The LIS problem is the only nontrivial example of the longest $Y$-avoiding subsequence problem with antipatterns of length 2. Albert et al. [4] gave the full classification of the longest $Y$-avoiding subsequence problem for all sets of antipatterns of length 3 . There are 10 non-trivial sets of such antipatterns. For each of these sets, the algorithms given in [4] run in polynomial time, ranging from $O(n \log n)$ to $O\left(n^{5}\right)$. Two particular antipattern sets considered in [4] are (in that work's original notation):

$$
\begin{aligned}
& C_{3}=\{\text { "132", "213", "321" }\} \\
& C_{4}=\{\text { "132", "213", "312" }\}
\end{aligned}
$$


For both these antipattern sets, algorithms given in [4] run in time $O\left(n^{2} \log n\right)$.

We now give new algorithms for the longest $C_{3}$ - and $C_{4}$-avoiding subsequence problems, improving on the above algorithms in running time.

Permutation strings that are $C_{3}$-avoiding are all cyclic rotations of an increasing permutation string. The longest such subsequence in the target string can be found by the algorithm for the cyclic LCS problem between permutations (Section 7.2), running in time $O\left(n \log ^{2} n\right)$.

Permutation strings that are $C_{4}$-avoiding are all obtained from an increasing permutation string by reversing some suffix. The longest such subsequence in the target string can be found as follows. Let the target string $t$ be over the alphabet $\langle 0: n\rangle$. First, we call the standard LIS algorithm on $t$, obtaining explicitly prefix-prefix LCS scores

$$
l c s\left(t 1\left(\hat{\imath}+\frac{1}{2}\right), i d 1\left(t(\hat{\imath})+\frac{1}{2}\right)\right)=l c s\left(t 1\left(\hat{\imath}-\frac{1}{2}\right), i d 1\left(t(\hat{\imath})-\frac{1}{2}\right)\right)+1
$$

for all $\hat{\imath} \in\langle 0: n\rangle$. Independently, we call Algorithm 4 on $t$ against the reverse identity permutation $\overline{i d}$, and use Theorem 1 to process its output into a data structure that allows efficient queries of all suffix-prefix LCS scores $l c s(t\rfloor k, \overline{i d}\lfloor l)$ for all $k, l \in[0: n]$. Finally, we obtain the solution to the longest $C_{4}$-avoiding subsequence problem as

$$
\max _{\hat{\imath} \in\langle 0: n\rangle}\left(l \operatorname{cs}\left(t 1\left(\hat{\imath}+\frac{1}{2}\right), i d 1\left(t(\hat{\imath})+\frac{1}{2}\right)\right)+l c s(t\rfloor\left(\hat{\imath}+\frac{1}{2}\right), \overline{i d}\left\lfloor\left(t(\hat{\imath})+\frac{1}{2}\right)\right)\right)
$$

The overall running time is dominated by the call to Algorithm 4, which runs in time $O\left(n \log ^{2} n\right)$.

\subsection{Longest piecewise monotone subsequences}

The classical LIS problem asks for the longest increasing (or, equivalently, decreasing) subsequence in a permutation string. A natural generalisation is to ask for the longest subsequence that consists of a constant number of monotone pieces. In particular, given a permutation string $a$ of length $n$, the longest $k$-increasing subsequence (respectively, longest $k$-modal subsequence) problem asks for the longest subsequence in $a$ that is a concatenation of at most $k$ sequences, all of which are increasing (respectively, alternate between increasing and decreasing). In the case of the longest $k$-modal subsequence problem, we assume without loss of generality that $k$ is even. Both problems can be solved as an instance of the LCS problem, comparing the input permutation string $a$ against string $i d^{k}$, i.e. the concatenation of $k$ copies of the identity permutation $i d$ (respectively, against string $(i d \overline{i d})^{k / 2}$, i.e. the concatenation of $k$ alternating copies of $i d$ and its reverse $\overline{i d})$. The resulting alignment dag is of size $n \times k n$, and contains $k n$ match cells. Using standard sparse LCS algorithms $[59,10]$, such an instance of the LCS problem can be 


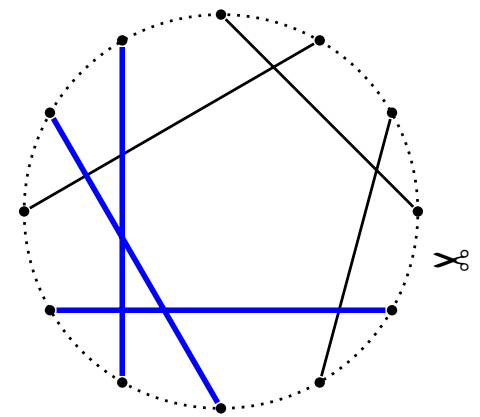

(a) The chord model

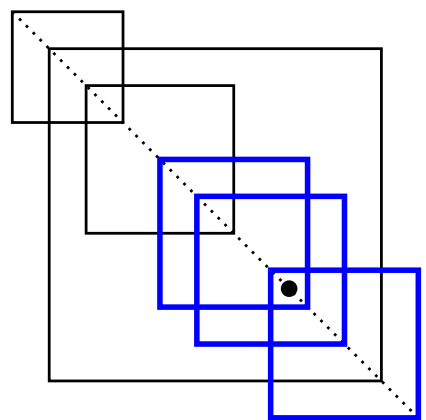

(b) An interval model

Figure 7.2: A circle graph and its maximum clique

solved in time $O(n k \log n)$. Demange et al. [38] gave a similar algorithm for the longest $k$-modal subsequence problem, also running in time $O(n k \log n)$.

We now give a new algorithm for the longest $k$-increasing subsequence and the longest $k$-modal subsequence problems, improving on the above algorithms in running time, unless $k$ is very small.

Our algorithm is as follows. In the case of the longest $k$-increasing subsequence problem, we run Algorithm 4, obtaining the semi-local seaweed ma$\operatorname{trix} P_{i d, a}$. Then, we extract the string-substring seaweed submatrix $P_{i d, a}^{s-s u b}$, and run on it $\log k$ times the algorithm of Theorem 10, obtaining the stringsubstring seaweed submatrix $P_{i d^{k}, a}^{s-s u b}$. In the case of the longest $k$-modal subsequence problem, we assume without loss of generality that $k$ is even. We run Algorithm 4 twice, obtaining the semi-local seaweed matrices $P_{i d, a}$ and $P_{\overline{i d}, a}$, from which we obtain the matrix $P_{i d \overline{i d}, a}$ by Theorem 12. Then, we extract the string-substring seaweed submatrix $P_{i d}^{s-s u b} \overline{i d}, a$, and run on it $\log k-1$ times the algorithm of Theorem 10, obtaining the string-substring seaweed submatrix $P_{(i d \overline{i d})^{k / 2}, a}^{s-s u b}$. Finally, for both problems we use the resulting string-substring seaweed submatrix to query the global LCS score. The described algorithm runs in time $O\left(n \log ^{2} n\right)+\log k \cdot O(n \log n)=O\left(n \log ^{2} n\right)$. This is an improvement on both the sparse LCS approach and the algorithm of [38], as long as $k \geq \log n$.

\subsection{Maximum clique in a circle graph}

A circle graph $[43,51]$ is defined as the intersection graph of a set of chords in a circle, i.e. the graph where each node represents a chord, and two nodes are adjacent, whenever the corresponding chords intersect. We consider the maximum clique problem on a circle graph.

The interval model of a circle graph is obtained by cutting the circle at an arbitrary point and laying it out on a line, so that the chords become (closed) intervals. The original circle graph is isomorphic to the overlap 
graph of its interval model, i.e. the graph where each node represents an interval, and two nodes are adjacent, whenever the corresponding intervals intersect but do not contain one another.

Example 21 Figure 7.2 shows an instance of the maximum clique problem on a six-node circle graph. Subfigure 7.2a shows the set of chords defining a circle graph, with one of the maximum cliques highlighted in bold blue. The cut point is shown by scissors. Subfigure $7.2 \mathrm{~b}$ shows the corresponding interval model; the dotted diagonal line contains the intervals, each defined by the diagonal of blue a square. The squares corresponding to the maximum clique are highlighted in bold blue.

It has long been known that the maximum clique problem in a circle graph on $n$ nodes is solvable in polynomial time [47]. A number of algorithms have been proposed for this problem [99, 57, 85, 9]; the problem has also been studied in the context of line arrangements in the hyperbolic plane $[64,41]$. Given an interval model of a circle graph, the running time of the above algorithms is $O\left(n^{2}\right)$ in the worst case, i.e. when the input graph is dense. In $[106,108]$, we gave an algorithm running in time $O\left(n^{1.5}\right)$.

We now give a new algorithm for the maximum clique problem in a circle graph, improving on existing algorithms in running time. The algorithm is based on the fast matrix distance multiplication procedure of Theorem 8 .

Our algorithm takes as input the interval model of a circle graph $G$ on $n$ nodes. Without loss of generality, we may assume that the set of interval endpoints is $\langle 0: 2 n\rangle$. The interval model is represented by a permutation string $a$ of size $2 n$, where for each left (respectively, right) interval endpoint $\hat{\imath} \in\langle 0: 2 n\rangle, a(\hat{\imath})$ is the corresponding right (respectively, left) endpoint. Note that for all $\hat{\imath}<\hat{\jmath}$, an interval with left endpoint $\hat{\imath}$ does not contain an interval with left endpoint $\hat{\jmath}$, if and only if $a(\hat{\imath})<a(\hat{\jmath})$. Various alternative representations of interval models (e.g. the ones used in $[99,9]$ ) can be converted to this representation in linear time.

In the interval model, a clique corresponds to a set of pairwise intersecting intervals, none of which contains another interval from the set. Recall that intervals in the line satisfy the Helly property: if all intervals in a set intersect pairwise, then they all intersect at a common point. In our context, we only need to consider integer indices as intersection points.

Consider a clique in $G$. Let $k \in[1: 2 n-1]$ be a common intersection point of the intervals representing the clique, which is guaranteed to exist by the Helly property. Since the intervals representing the clique cannot contain one another, the sequence of their right endpoints is an increasing subsequence of $a$. Let $i d$ be the identity permutation string of length $2 n$. From the observations above, it follows that the clique corresponds to a common subsequence of a prefix $a \uparrow k$ and a suffix $i d\rfloor k$. Therefore, the maximum clique can be solved as an instance of the semi-local LCS problem. 


\section{Algorithm 5 (Maximum clique in a circle graph)}

Input: interval model of circle graph $G$, represented by permutation string $a$ of size $2 n$.

Output: maximum-size clique of $G$, represented by the set of (say) left endpoints of the corresponding intervals.

\section{Description.}

First phase. We run Algorithm 4 on the input permutation string $a$ against the identity permutation string $i d$, obtaining a semi-local seaweed matrix of size $4 n$. We then build the data structure of Theorem 1 for querying semi-local LCS scores of $a$ against $i d$.

Second phase. For each $k \in[1: 2 n-1]$, we query the LCS score of prefix $a 1 k$ against suffix $i d\rfloor k$. The maximum of the $2 n$ returned values gives the size of the maximum clique in $G$, and the corresponding value $k$ gives a common intersection point of the clique intervals.

Third phase. The intervals defining the maximum clique can now be obtained by running a standard LIS algorithm on string $\left.(a 1 k)\right|_{\Sigma(i d\rfloor k)}$.

\section{Cost analysis.}

First phase. The running time of Algorithm 4 is $O\left(n \log ^{2} n\right)$.

Second phase. By Theorem 1, the combined running time of all the prefixsuffix queries is $O\left(n \log ^{2} n\right)$, if the queries are performed independently. This time can be reduced to $O(n)$ by observing that the queries can be performed as a single diagonal batch query.

Third phase. The LIS algorithm runs in time $O(n \log n)$.

Total. The overall running time is $O\left(n \log ^{2} n\right)$.

Like many algorithmic problems, the problem of finding a maximum clique in a circle graph admits various parameterised versions. Some relevant parameters are:

- the size $l$ of the maximum clique;

- the thickness $d$ of the interval model, i.e. the maximum number of intervals containing a point, taken across all points in the line;

- the number $e$ of graph edges.

For any interval model of a non-trivial circle graph, we have $l \leq d \leq n \leq$ $e \leq n^{2}$. Notice that, given a permutation representing an interval model, its thickness can be found in time $O\left(n \log ^{2} n\right)$ by building a range tree on the corresponding set of planar points, and then performing $O(n)$ dominance counting queries. 
Apostolico et al. [9] give algorithms for the parameterised version of the maximum clique problem in a circle graph, running in time $O(n \log n+e)$ and $O(n \log n+n l \log (n / l))$. They also describe an algorithm for the maximum independent set problem, parameterised by the interval model's thickness.

We now give a new algorithm for the maximum clique problem in a circle graph, parameterised by the thickness of the input interval model. Our algorithm improves on the parameterised algorithms of [9] for most values of the parameters. The algorithm is an extended version of Algorithm 5.

\section{Algorithm 6 (Maximum clique in a circle graph, parameterised by thickness)}

Input: interval model of circle graph $G$, represented by string $a$ of size $2 n$.

Output: maximum-size clique of $G$, represented by the set of (say) left endpoints of the corresponding intervals.

Parameter: thickness $d, d \leq n$, of the input interval model.

\section{Description.}

First phase. We run Algorithm 4 on string $a 1(r+1)$ against string $i d\rfloor r d$, independently for all $r \in\left[0: \frac{2 n}{d}-1\right]$. As will be shown in the algorithm's analysis, in each run we obtain an semi-local seaweed matrix with at most $4 d$ non-trivial nonzeros. For every $r$, we then build the data structure of Theorem 1 for querying semi-local LCS scores of $a 1(r+1)$ against $i d \downarrow r d$.

Second phase. For each $k \in[1: 2 n-1]$, we query the LCS score of prefix $a 1 k=(a 1(r+1) d) 1 k$ against suffix $i d\rfloor k=(i d\rfloor r d)\rfloor(k-r d)$, where $r=\lfloor k / d\rfloor$. The maximum of the $2 n$ returned values gives the size of the maximum clique in $G$, and the corresponding value $k$ gives a common intersection point of the clique intervals.

Third phase. The intervals defining the maximum clique can now be obtained by running a standard LIS algorithm on string $\left.(a 1 k)\right|_{\Sigma(i d\rfloor k)}$.

\section{Cost analysis.}

First phase. We have $a 1(r+1) d=(a \uparrow r d)((a\rfloor r d) 1 d)$. The alignment dag of $a 1 r d$ against $i d \downarrow r d$ contains at most $d$ match cells, since every match corresponds to an interval containing point $r d$, and there can be at most $d$ such intervals by the definition of thickness. The alignment dag of $(a\rfloor r d) 1 d$ against $i d\rfloor r d$ also contains at most $d$ match cells, since the length of the string $(a\rfloor r d) 1 d$ is $d$. The alignment dag of $a 1(r+1) d$ against $i d\rfloor r d$ is the composition of the above two alignment dags, and therefore contains at most $d+d=2 d$ matches. Therefore, the time for each run of Algorithm 4 is $O\left(d \log ^{2} d\right)$, and the overall running time of this phase is $O\left(n / d \cdot d \log ^{2} d\right)=$ $O\left(n \log ^{2} d\right)$.

Second phase. By Theorem 1, the combined running time of all the prefixsuffix queries is $O\left(n \log ^{2} d\right)$, if the queries are performed independently. This 
time can be reduced to $O(n / d \cdot d)=O(n)$ by observing that the queries can be performed as a single diagonal batch query.

Third phase. The alignment dag of $a 1 k$ against $i d\rfloor k$ contains at most $d$ matches, since every such match corresponds to an interval containing point $k$. Therefore, string $\left.(a \nmid k)\right|_{\Sigma(i d\rfloor k)}$ has length at most $d$. The LIS algorithm runs in time $O(d \log d)$.

Total. The resulting overall running time is $O\left(n \log ^{2} d\right)$.

Algorithm 6 improves on the $O(n \log n+e)$ algorithm of [9], unless $e=$ $o\left(n \log ^{2} d\right)=O\left(n \log ^{2} n\right)$. It also improves on the $O(n \log n+n l \log (n / l))$ algorithm of [9], unless $l=o\left(\frac{\log ^{2} d}{\log n}\right)=O(\log n)$.

\subsection{Maximum common pattern between linear graphs}

The concept of a linear graph, introduced by Davydov and Batzoglou [37], is similar to an interval model of a circle graph defined in Section 7.5. Fertin et al. [44] considered the problem of finding the maximum common pattern in a set of $n$ linear graphs, each defined by at most $m$ intervals. Common patterns are defined as ordered subsets of intervals that are isomorphic with respect to interval disjointness, containment and overlapping. These three interval properties are denoted respectively by symbols $<, \sqsubset$ and $\gamma$. The structure of the common pattern may be restricted by only considering patterns where the intervals must pairwise satisfy a prescribed subset of these properties. The resulting maximum common $S$-structured pattern ( $S-M C S P$ ) problem is parameterised by the nonempty set $S \subseteq\{<, \sqsubset, \varnothing\}$ of prescribed properties. In particular, the $\{\chi\}$-MCSP problem asks for the maximum commonly-structured subset of pairwise overlapping intervals; for $n=1$ this is equivalent to finding the maximum clique of a circle graph, and for general $n$ is equivalent to finding the minimum-sized clique among maximum cliques of the $n$ input circle graphs. The $\{<, \sqsubset\}$-MCSP problem asks for the maximum commonly-structured subset of intervals, no two of which are overlapping; for $n=1$ this is equivalent to finding the maximum independent set of a circle graph; however, for general $n$ the maximum commonly-structured independent set of the $n$ input circle graphs may be significantly different from (and smaller than) each of the $n$ individual maximum independent sets. The $\{<, \sqsubset, \varnothing\}$-MCSP problem asks for the maximum commonly-structured subset of intervals without any a priori restriction on its structure.

Extending and generalising a number of previous results, paper [44] considers the $S$-MCSP problem for each of seven nonempty subsets of $\{<, \sqsubset, \varnothing\}$. For some of these seven variants, the algorithms use as a subroutine the algorithm of $[106,108]$ for the maximum clique problem in a circle graph. By plugging in the more efficient Algorithm 5, we can obtain improved 
algorithms for those variants of the $S$-MCSP problem, where finding the maximum clique in a circle graph is a bottleneck.

In particular, the $\{\chi\}$-MCSP problem is solved in [44] by finding the maximum clique independently for $n$ circle graphs, each corresponding to one of the input linear graphs, in overall time $O\left(n m^{1.5}\right)$. By plugging in Algorithm 5, the running time is improved to $O\left(n m \log ^{2} m\right)$.

The $\{<, \chi\}$-MCSP problem is shown in [44] to be NP-hard, and to admit a polynomial-time $h(k)$-approximation, where $h(k)=\sum_{1 \leq i \leq k} 1 / i$; for the rest of this section, $k$ denotes the size of the solution. The approximation is obtained by finding the maximum clique for $n m \log m$ different circle graphs, in overall time $O\left(n m^{2.5} \log ^{2} m\right)$. By plugging in Algorithm 5, the running time of the approximation algorithm is improved to $O\left(n m^{2} \log ^{4} m\right)$.

The $\{\sqsubset, \varnothing\}$-MCSP problem is also shown in [44] to be NP-hard, and to admit a polynomial-time $k^{1 / 2}$-approximation. The approximation is obtained by combining exact solutions for the $\{\sqsubset\}$-MCSP and $\{\varnothing\}$-MCSP problems on the same input sets. The exact solution for the $\{\varnothing\}$-MCSP is the bottleneck; by plugging in the improved algorithm for this problem described above, the running time of the approximation algorithm for the $\{\sqsubset, \varnothing\}$-MCSP problem is improved from $O\left(n m^{1.5}\right)$ to $O\left(n m \log ^{2} m\right)$.

Finally, paper [44] argues that the $\{<, \sqsubset, \chi\}$-MCSP problem is NP-hard, and gives several polynomial-time approximation algorithms. In particular, it gives an $O\left(k^{2 / 3}\right)$-approximation algorithm running in time $O\left(\mathrm{~nm}^{1.5}\right)$, and an $O\left((k \log k)^{1 / 2}\right)$-approximation algorithm running in time $O\left(n m^{2.5} \log m\right)$. Again, the exact solution for the $\{\varnothing\}$-MCSP is the bottleneck; by plugging in the improved algorithm, the running times of the approximation algorithms improve respectively to $O\left(n m \log ^{2} m\right)$ and $O\left(n m^{2} \log ^{4} m\right)$. 


\section{Chapter 8}

\section{Compressed string comparison}

\subsection{Grammar-compressed strings}

Algorithms on compressed strings is an area of steadily increasing importance in algorithm theory. Early examples of such algorithms were given e.g. by Amir et al. [8] and by Rytter [100].

Definition 25 Let $t$ be a string of length $n$ (typically large). A grammarcompressed string (GC-string) is a special type of context-free grammar, called a straight-line program (SLP), which generates $t$. An SLP of length $\bar{n}, \bar{n} \leq n$, is a sequence of $\bar{n}$ statements. A statement numbered $k, 1 \leq k \leq \bar{n}$, has one of the following forms:

$$
\begin{array}{ll}
t_{k}=\alpha & \text { where } \alpha \text { is an alphabet character } \\
t_{k}=t_{i} t_{j} & \text { where } 1 \leq i, j<k
\end{array}
$$

We identify every symbol $t_{r}$ with the string it represents; in particular, we have $t=t_{\bar{n}}$. In general, the plain string length $n$ can be exponential in the GC-string length $\bar{n}$.

Example 22 The Fibonacci string "abaababaabaab" of length 13 can be represented by the following SLP of length 7:

$$
\begin{array}{ll}
t_{1}=\text { 'b' } & t_{2}=\text { 'a' } \\
t_{3}=t_{2} t_{1} \quad t_{4}=t_{3} t_{2} \quad t_{5}=t_{4} t_{3} \quad t_{6}=t_{5} t_{4} \quad t_{7}=t_{6} t_{5}
\end{array}
$$

In general, a Fibonacci string of length $n$ can be represented by an SLP of length $\bar{n}$, where $n=F_{\bar{n}}=\left(\frac{1}{\sqrt{5}}-o(1)\right)\left(\frac{1+\sqrt{5}}{2}\right)^{\bar{n}}$ is the $\bar{n}$-th Fibonacci number.

This example is borrowed from Hermelin et al. [55]. 
Grammar compression includes as a special case the well-known LZ78 and LZW compression schemes by Ziv, Lempel and Welch [117, 114]. Both these schemes can be expressed by an SLP that consists of three sections:

- in the first section, all statements are of the form $t_{k}=\alpha$;

- in the second section, all statements are of the form $t_{k}=t_{i} t_{j}$, where statement $j$ is from the first section;

- in the third section, all statements are of the form $t_{k}=t_{k-1} t_{j}$, where statement $j$ is from the second section.

It should also be noted that certain other compression methods, such as e.g. LZ77 [116] and run-length compression, do not fit directly into the grammar compression model.

The algorithms in this section will take as input a text string $t$ of length $n$, represented by an SLP of length $\bar{n}$, and a pattern string $p$ of length $m$, represented explicitly. We aim at algorithms with running time that is a low-degree polynomial in $m, \bar{n}$, but is independent of $n$ (which could be exponential in $\bar{n}$ ).

For brevity, a substring of $t$ containing $p$ as a subsequence will be called $p$-matching. A $p$-matching substring will be called minimally $p$-matching, if it has no proper $p$-matching substring.

\subsection{Global subsequence recognition}

The global subsequence recognition problem has been defined in Section 3.1; it is a simple special case of the LCS problem. In this section, we revisit this problem, now considering the input text to be grammar-compressed.

The global subsequence recognition problem asks whether the whole text $t$ is $p$-matching. Global subsequence recognition on a GC-text can be performed by the following simple folklore algorithm.

\section{Algorithm 7 (Global subsequence recognition)}

Input: SLP of length $\bar{n}$, generating text string $t$ of length $n$; plain pattern string $p$ of length $m$.

Output: an integer $k$, giving the length of the longest prefix of $p$ that is a subsequence of $t$. String $t$ contains $p$ as a subsequence, if and only if $k=m$.

Description. Recursion on the input SLP generating $t$.

Recursion base. If $\bar{n}=n=1$, then the output value $k \in\{0,1\}$ is determined by a single character comparison.

Recursive step. Let $t=t^{\prime} t^{\prime \prime}$ be the SLP statement defining string $t$. Let $k^{\prime}$ be the length of the longest prefix of $p$ that is a subsequence of $t^{\prime}$. Let $k^{\prime \prime}$ be the length of the longest prefix of $p\rfloor k^{\prime}$ that is a subsequence of $t^{\prime \prime}$. We call the algorithm recursively to obtain $k^{\prime}$ and $k^{\prime \prime}$, and then return $k=k^{\prime}+k^{\prime \prime}$. 
End of recursive step.

Cost analysis. The running time of the algorithm is $O(k \bar{n})$. The proof is by induction. The running time of the recursive calls is respectively $O\left(k^{\prime} \bar{n}\right)$ and $O\left(k^{\prime \prime} \bar{n}\right)$. The overall running time of the algorithm is $O\left(k^{\prime} \bar{n}\right)+O\left(k^{\prime \prime} \bar{n}\right)+$ $O(1)=O(k \bar{n})$. In the worst case, this is $O(m \bar{n})$.

\subsection{Three-way semi-local LCS}

We recall from Chapter 5 that the LCS problem on a pair of plain strings can be solved in time $O\left(\frac{m n}{\log ^{2} n}\right)$, assuming that $m \leq n$ and that $m$ and $n$ are reasonably close, by the micro-block method of Masek and Paterson [84]. The LCS problem on a pair of GC-strings has been considered by Lifshits and Lohrey [80], and proven to be NP-hard.

Recall that we aim at algorithms with running time independent of $n$ (which could be exponential in $\bar{n}$ ). This rules out any attempt at solving the full semi-local LCS problem, since the resulting semi-local seaweed matrix would require memory $O(m+n)$. However, we are still able to consider the three-way semi-local LCS problem, excluding the computation of LCS on substrings of $t$.

The described version of the LCS problem can be regarded as a special case of the edit distance problem between a context-free language given by a grammar of size $\bar{n}$, and a string of size $m$. For this more general problem, Myers [88] gave an algorithm running in time $O\left(m^{3} \bar{n}+m^{2} \cdot \bar{n} \log \bar{n}\right)$. In [109], we gave an algorithm for the three-way semi-local LCS problem, running in time $O\left(m^{1.5} \bar{n}\right)$. Lifshits [79] asked whether the LCS problem can be solved in time $O(m \bar{n})$.

A new algorithm for the three-way semi-local LCS problem can be obtained by plugging into our previous algorithm [109] the fast matrix distance multiplication procedure of Theorem 8 . The resulting algorithm improves on existing algorithms in running time, and approaches an answer to Lifshits' question within a logarithmic factor.

\section{Algorithm 8 (Three-way semi-local LCS)}

Input: SLP of length $\bar{n}$, generating text string $t$ of length $n$; plain pattern string $p$ of length $m$.

Output: nonzeros of prefix-suffix, suffix-prefix and substring-string seaweed matrices $P_{p, t}^{p x-s x}, P_{p, t}^{s x-p x}, P_{p, t}^{s u b-s}$.

\section{Description.}

First phase. Recursion on the input SLP generating $t$. To avoid an exponential blow-up of the indices, matrix range remapping is performed in every recursive call, while preserving the index ordering of the nonzeros. 
Recursion base. If $\bar{n}=n=1$, then the output seaweed submatrices can be computed by a linear sweep of string $p$.

Recursive step. Let $t=t^{\prime} t^{\prime \prime}$ be the SLP statement defining string $t$. We call the algorithm recursively to obtain seaweed matrices

$$
\begin{array}{ccc}
P_{p, t^{\prime}}^{p x-s x} & P_{p, t^{\prime}}^{s x-p x} & P_{p, t^{\prime}}^{s u b-s} \\
P_{p, t^{\prime \prime}}^{p x-s x} & P_{p, t^{\prime \prime}}^{s x-p x} & P_{p, t^{\prime \prime}}^{s u b-s}
\end{array}
$$

The total number of nonzeros in each matrix triple is at most $m$. Conceptually, these matrices are submatrices of the semi-local seaweed matrices $P_{p, t^{\prime}}$, $P_{p, t^{\prime \prime}}$ over $\left\langle-m: n \mid 0: m+n^{\prime}\right\rangle,\left\langle-m: n \mid 0: m+n^{\prime \prime}\right\rangle$, respectively. The remapped ranges of the matrices are $\langle-m: m \mid 0: 2 m\rangle$. We now compute the composition seaweed matrices

$$
P_{p, t}^{p x-s x} \quad P_{p, t}^{s x-p x} \quad P_{p, t}^{s u b-s}
$$

by Corollary 2. The total number of nonzeros in the three matrices is still at most $m$. Conceptually, these matrices are submatrices of the semi-local seaweed matrix $P_{p, t}$ over $\langle-m: n \mid 0: m+n\rangle$. Taking into account the range remapping of $P_{p, t^{\prime}}, P_{p, t^{\prime \prime}}$, the resulting range of $P_{p, t}$ is $\langle-2 m: m \mid 0: 3 m\rangle$. We now perform another step of remapping, transforming the range of the three component submatrices of $P_{p, t}$ to $\langle-m: m \mid 0: 2 m\rangle$, while preserving the index ordering of the nonzeros.

End of recursive step.

Second phase. We now have the nonzeros of the output matrices, remapped to the range $\langle-m: m \mid 0: 2 m\rangle$. If explicit indices of the nonzeros in the (possibly exponentially larger) range $\langle-m: n \mid 0: m+n\rangle$ are required, they can be obtained by reversing each remapping step recursively, following the structure of the SLP representing string $t$.

\section{Cost analysis.}

First phase. By Corollary 2, each recursive step runs in time $O(m \log m)$. There are $\bar{n}$ recursive steps in total, therefore the first phase runs in time $O(m \log m \cdot \bar{n})$.

Second phase. For each nonzero, the inverse remapping can be performed recursively in time $O(\bar{n})$. There are $m$ nonzeros in total, therefore the second phase runs in time $O(m \bar{n})$.

Total. The overall running time is $O(m \log m \cdot \bar{n})$.

Algorithm 8 provides, as a special case, an algorithm for the LCS problem between a GC-string and a plain string, running in time $O(m \log m \cdot \bar{n})$; the LCS score can easily be queried from the algorithm's output by Theorem 9. This running time should be contrasted with standard LCS algorithms on plain strings, running in time $O\left(\frac{m n}{\log (m+n)}\right)$ [84, 34], and with the NP-hardness of the LCS problem on two GC-strings [80]. 
Hermelin et al. [55] gave a more detailed analysis of this problem's complexity, by considering the weighted alignment problem on a pair of GCstrings $a, b$ of total compressed length $\bar{r}=\bar{m}+\bar{n}$, parameterised by the strings' total plain length $r=m+n$. They gave an algorithm running in time $O\left(r^{1.34} \bar{r}^{1.34}\right)$ for general weights, and in time $O\left(r^{1.2} \bar{r}^{1.4}\right)$ for rational weights.

In the case of rational weights, the parameterised running time of weighted GC-string alignment can be improved by the following straightforward algorithm. First, we uncompress one of the input strings - say, string $b$. Then, we run Algorithm 8 on the GC-string $a$ against the plain version of string $b$. The resulting running time is $O(m \log m \cdot \bar{n})=O(r \log r \cdot \bar{r})$.

\subsection{Local subsequence recognition}

In Section 5.4, we considered local (minimal-window and fixed-window) subsequence recognition problems; they are special cases of both the semi-local LCS problem, and the approximate matching problem. The minimumwindow subsequence recognition problem asks for the locations of all substrings of $t$ that are minimally $p$-matching, and the fixed-window subsequence recognition problem asks for the locations of all the $p$-matching substrings of a fixed length $w$. A combination of these two problems is the bounded minimal-window subsequence recognition problem, which asks for the locations of all the minimally $p$-matching substrings below a fixed length $w$. Clearly, the output size for these reporting versions of the problems may be exponential in $\bar{n}$; therefore, we have to parameterise the running time by the output size. We will also consider the counting versions of the problems, that, instead of asking the precise locations of $p$-matching substrings in question, only ask for their overall number.

The minimal-window, fixed-window and bounded minimal-window subsequence recognition problems for a GC-text against a plain pattern have been considered by Cégielski et al. [24]. They gave algorithms that run in time $O\left(m^{2} \log m \cdot \bar{n}+\right.$ output $)$ for the reporting versions, and $O\left(m^{2} \log m \cdot \bar{n}\right)$ for the counting versions of the problems.

We now give a more efficient local subsequence recognition algorithm, based on Algorithm 8. In addition to the seaweed matrices $P_{p, t}^{p x-s x}, P_{p, t}^{s x-p x}$, $P_{p, t}^{s u b-s}$, we now also make use of the overlap submatrix $P_{p, t}^{s-o s u b}$.

\section{Algorithm 9 (Local subsequence recognition)}

Input: SLP of length $\bar{n}$, generating text string $t$ of length $n$; plain pattern string $p$ of length $m$.

Output: locations, or count, of minimally $p$-matching substrings in $t$.

Description.

First phase. We run Algorithm 8, extending every recursive step by the 
reporting of minimally $p$-matching substrings that are overlap substrings in the current seaweed matrix composition. Similarly to Algorithm 8, index remapping is performed in the background in order to avoid exponential index blow-up. To simplify the exposition, we now keep the remapping implicit.

Recursion base. As in Algorithm 8. If $\bar{n}=n=1$, then no substring of $t$ can be $p$-matching (unless $m=1$, in which case the whole problem is trivial).

Recursive step. Let $t=t^{\prime} t^{\prime \prime}$ be the SLP statement defining string $t$. We call the algorithm recursively to obtain seaweed matrices

$$
\begin{array}{ccc}
P_{p, t^{\prime}}^{p x-s x} & P_{p, t^{\prime}}^{s x-p x} & P_{p, t^{\prime}}^{s u b-s} \\
P_{p, t^{\prime \prime}}^{p x-s x} & P_{p, t^{\prime \prime}}^{s x-p x} & P_{p, t^{\prime \prime}}^{s u b-s}
\end{array}
$$

and then compute the composition seaweed matrices

$$
P_{p, t}^{p x-s x} P_{p, t}^{s x-p x} \quad P_{p, t}^{s u b-s}
$$

as well as the overlap substring-string seaweed submatrix

$$
P_{p, t^{\prime}, t^{\prime \prime}}^{o s u b-s}
$$

by Corollary 2.

A substring $t\langle i: k\rangle$ is $p$-matching, if and only if the point $(i, k)$ is not $\lessgtr$-dominated by any nonzeros in the seaweed matrix $P_{p, t}$, or, equivalently, is not $\lessgtr$-dominated by any of its $\lessgtr$-maximal nonzeros. Recall that a substring $t\langle i: k\rangle$ is an overlap substring, if $i \in\left[0: n^{\prime}-1\right], k \in\left[n^{\prime}+1: n\right]$; in other words, an overlap substring consists of a non-empty suffix of $t^{\prime}$ and a nonempty prefix of $t^{\prime \prime}$. For an overlap substring $t\langle i: k\rangle$, any nonzero in $P_{p, t}$ that $\lessgtr$-dominates point $(i, k)$ must belong to the disjoint matrix union

$$
\tilde{P}_{p, t^{\prime}, t^{\prime \prime}}=P_{p, t}^{s u b-s} \sqcup P_{p, t}^{p x-s x} \sqcup P_{p, t}^{s x-p x} \sqcup P_{p, t^{\prime}, t^{\prime \prime}}^{s-o s b}
$$

Matrix $\tilde{P}_{p, t^{\prime}, t^{\prime \prime}}$ has at most $m$ nonzeros. Consider the $\ll$-chain of all $\lessgtr-$ maximal nonzeros in $\tilde{P}_{p, t^{\prime}, t^{\prime \prime}}$, and denote them by

$$
\left(\hat{\imath}_{0}, \hat{k}_{0}\right) \ll\left(\hat{\imath}_{1}, \hat{k}_{1}\right) \ll \cdots \ll\left(\hat{\imath}_{s-1}, \hat{k}_{s-1}\right)
$$

where $s, 1 \leq s \leq m$, is the total number of $\lessgtr$-maximal nonzeros in $\tilde{P}_{p, t^{\prime}, t^{\prime \prime}}$. Let $i \in\left[0: n^{\prime}-1\right], k \in\left[n^{\prime}+1: n\right]$. An overlap substring $t\langle i: k\rangle$ is minimally $p$-matching, if and only if $(i, k)$ is one of the $s-1$ points in the "interpolated" $\ll$-chain

$$
\left(\hat{\imath}_{1}-\frac{1}{2}, \hat{k}_{0}+\frac{1}{2}\right) \ll\left(\hat{\imath}_{2}-\frac{1}{2}, \hat{k}_{1}+\frac{1}{2}\right) \ll \cdots \ll\left(\hat{\imath}_{s-1}-\frac{1}{2}, \hat{k}_{s-2}+\frac{1}{2}\right)
$$

Therefore, the number of minimally $p$-matching overlap substrings can be obtained as a count of points in the above «-chain having $i \in\left[0: n^{\prime}-1\right]$, $k \in\left[n^{\prime}+1: n\right]$. This count is always at most $s-1 \leq m-1$.

End of recursive step. 


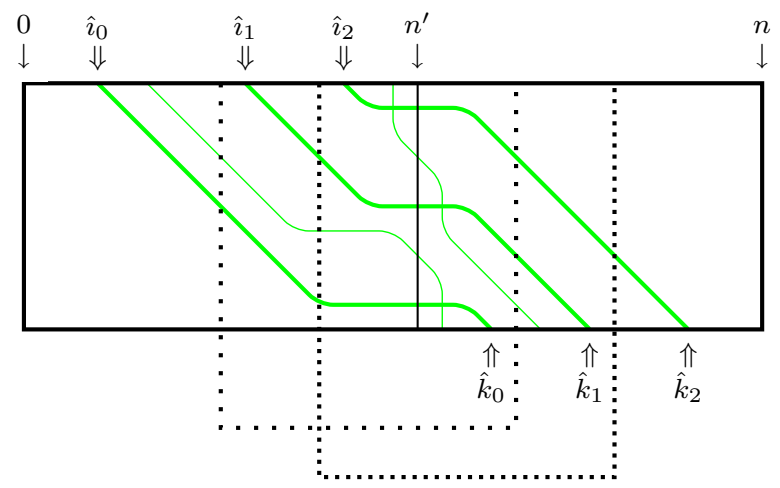

Figure 8.1: A snapshot of Algorithm 9 (local subsequence recognition)

Second phase. For every SLP symbol, we now have the locations of its minimally $p$-matching overlap substrings, of which there are at most $m-1$. By another recursion on the structure of the SLP, it is now straightforward to obtain either the locations or the count of all the minimally $p$-matching substrings in $t$.

\section{Cost analysis.}

First phase. As in Algorithm 8, each seaweed matrix composition runs in time $O(m \log m)$. The «-chain of $\lessgtr$-maximal nonzeros in matrix $\tilde{P}_{p, t^{\prime}, t^{\prime \prime}}$ can be found in time $O(m)$. Hence, the running time of a recursive step is $O(m \log m)$. There are $\bar{n}$ recursive steps in total, therefore the whole recursion runs in time $O(m \log m \cdot \bar{n})$.

Second phase. Given the output of the first phase, the locations of all $p$ matching substrings in $t$ can now be found in time $O(m \bar{n}+$ output $)$; the count of such substrings can be obtained in time $O(m \bar{n})$.

Total. The overall running time is $O(m \log m \cdot \bar{n}+$ output $)$ for the reporting version, and $O(m \log m \cdot \bar{n})$ for the counting version.

Example 23 Figure 8.1 shows a snapshot of a recursive step in the first phase of Algorithm 9. Nonzeros of the matrix $\tilde{P}_{p, t^{\prime}, t^{\prime \prime}}$ are shown by green seaweeds. In this particular example, $P_{t, p}^{s u b-s}, P_{t, p}^{p x-s x}, P_{t, p}^{s x-p x}$ are all zero matrices, so the nonzeros of $\tilde{P}_{p, t^{\prime}, t^{\prime \prime}}$ are in fact the nonzeros of $P_{p, t^{\prime}, t^{\prime \prime}}^{s-s u b}$. There are $m=5$ such nonzeros, of which $s=3$ are $\lessgtr$-maximal, as shown by thick seaweeds. Therefore, there are $s-1=2$ minimally $p$-matching overlap substrings in $t$, shown by dotted brackets. Visually, an overlap substring in $t$ is $p$-matching, if and only if the corresponding rectangle in the alignment dag is not pierced by a seaweed entering at the left-hand boundary and leaving at the right-hand boundary. Notice that the bracketed substrings of $t$ in Figure 8.1 are exactly the two inclusion-minimal overlap substrings satisfying this property. 
An algorithm for the fixed-window subsequence recognition problem can be obtained from Algorithm 9 as follows. Substrings $t\langle i: k\rangle$ of length $w$ correspond to points lying on the diagonal $k-i=w$ in the semi-local score matrix $H_{p, t}$. Consider the «-chain of all $s \leq n$ nonzeros that are $\lessgtr-$ maximal in the matrix $\tilde{P}_{p, t^{\prime}, t^{\prime \prime}}$, computed by Algorithm 9. An overlap substring $t\langle i: k\rangle, k-i=w$, is $p$-matching, if and only if $i$ belongs to the (not necessarily disjoint) union of $s-1$ intervals

$$
\begin{aligned}
& {\left[\hat{k}_{0}+\frac{1}{2}-w: \hat{\imath}_{1}-\frac{1}{2}\right] \cup\left[\hat{k}_{1}+\frac{1}{2}-w: \hat{\imath}_{2}-\frac{1}{2}\right] \cup \cdots \cup} \\
& \quad\left[\hat{k}_{s-3}+\frac{1}{2}-w: \hat{\imath}_{s-2}-\frac{1}{2}\right] \cup\left[\hat{k}_{s-2}+\frac{1}{2}-w: \hat{\imath}_{s-1}-\frac{1}{2}\right]
\end{aligned}
$$

where any interval of negative length is by convention considered empty. In every recursive step, the interval endpoints can be computed in time $O(n)$; after that, each interval element corresponding to an overlap substring of $t$ can be reported in constant time.

An algorithm for the bounded minimal-window subsequence recognition problem can be obtained from Algorithm 9 by discarding in every recursive step the minimally $p$-matching substrings of length exceeding $w$.

The overall running time of both the above modifications of Algorithm 9 is still $O(m \log m \cdot \bar{n}+$ output $)$ for the reporting versions, and $O(m \log m \cdot \bar{n})$ for the counting versions. 


\section{Chapter 9}

\section{Beyond semi-locality}

\subsection{Window-local LCS and alignment plots}

So far, we have considered mostly global and semi-local string comparison. Our aim now is to approach local string comparison - the type of string comparison that is the most important for biological applications, but also the most difficult. In this chapter, we consider a version of local string comparison that is restricted to a fixed subset of prescribed substrings in one of the input strings, comparing them to all substrings in the other string.

An important special case is where all the prescribed substrings have equal length. Given a fixed parameter $w$, we call a substring of length $w$ a window in the corresponding string.

String comparison in windows has a long history. One of its early instances is dot plots (also known as diagonal plots or dot matrices), introduced by Gibbs and McIntyre [50] and by Maizel and Lenk [82]. In addition to numerical scores, dot plots provide a convenient visualisation of string comparison. In the context of dot plots, processing a pair of windows is usually referred to as filtering. The standard filtering method compares every window of string $a$ against every window of string $b$ in terms of their Hamming score, i.e. the count of matching characters along the main diagonal of the windows' Cartesian product. A Hamming-filtered dot plot can be computed in time $O(m n)$ by the algorithm of $[82,86]$. This algorithm has been implemented in several software packages (see e.g. [104, 95, 30]). A faster suffix-tree based algorithm has been proposed and implemented by Krumsiek et al. [71]. Enhancement of the dot plot approach have been proposed by Huang and Zhang [58] and by Putonti et al. [94].

Numerous other methods of local string comparison have been proposed. The Smith-Waterman-Gotoh algorithm [103, 53] allows one to obtain the highest-scoring pair across all substring pairs in the input strings. It can also be generalised to report all substring pairs scoring above a certain threshold. A significant drawback of the Smith-Waterman-Gotoh algorithm is that it 
generally favours long, less precise substring alignments over short, more precise ones (as noted e.g. by Arslan et al. [13]). The quality of the alignment is also dependent on the scoring scheme: for example, for the LCS score, the algorithm only provides the trivial global comparison, so the method is generally only useful for weighted alignment scores with sufficiently high penalties (negative score weights) for gaps.

In contrast with the Smith-Waterman-Gotoh algorithm, the dot plot method gives the user more flexibility to select the biologically significant substring alignments, by providing all the local scores between fixed-size windows of the input strings. However, the Hamming scoring scheme used by this method within each window pair is less sensitive than even the LCS score, and especially than the weighted alignment score used by SmithWaterman-Gotoh. This tradeoff motivates us to combine the best features of the two approaches in the following definition.

Definition 26 Given strings $a, b$, the window-window (respectively, windowsubstring) LCS problem asks for the LCS score of for every window in $a$ against every window (respectively, substring) in $b$.

The window-window LCS problem can be seen as a refinement of the dot plot method and a complement to the Smith-Waterman-Gotoh method. As in the dot plot method, we compute all window-window comparison scores between the input strings. However, instead of the Hamming score, our method is based on the LCS score, and is therefore potentially more sensitive. The method can be further extended to use weighted alignment scores. By analogy with Hamming-filtered dot plots, we call the resulting matrix of window-window alignment scores an alignment-filtered dot plot, or simply an alignment plot. A similar method has been proposed recently for detection of alignment-conserved regions in DNA [91].

The solution of the window-substring LCS problem can be represented in space $O(m n \log n)$ by the data structure of Theorem 1 , built on the stringsubstring seaweed submatrix for each window of $a$ against $b$. An individual window-substring LCS score query can be performed on this data structure by Theorem 1 in time $O\left(\log ^{2} n\right)$. The full explicit solution to the windowwindow LCS problem can be obtained in time $O(m n)$, by performing a diagonal batch query directly on each of the string-substring seaweed submatrices. Thus, string-substring seaweed submatrices provide a unified solution for both the window-substring and the window-window LCS problems.

A naive algorithm for the window-window LCS problem runs in time $O\left(m n w^{2}\right)$, and for the window-substring LCS problem runs in time $O\left(m n^{3} w\right)$. The running time for both problems can be improved by applying Algorithm 1 (the seaweed algorithm) independently to each window of string $a$ against whole string $b$. The resulting algorithm runs in time $O(m n w)$. If window length $w$ is sufficiently large, the running time can be improved slightly by using Algorithm 2 (the micro-block seaweed algorithm). 
We now give a new algorithm for the window-substring LCS problem (and therefore also for the window-window LCS problem as a special case). Our algorithm provides a further substantial improvement on the above approach, and matches the asymptotic running time of both the Hammingscored dot plot and the Smith-Waterman-Gotoh methods.

\section{Algorithm 10 (Window-substring LCS)}

Input: strings $a, b$ of length $m, n$, respectively; window length $w$.

Output: nonzeros of the string-substring seaweed submatrix for every window of string $a$ against full string $b$.

Description. For simplicity, we assume that $m$ is a power of 2 . We call a substring of the form $a\left\langle r \cdot 2^{s}:(r+1) \cdot 2^{s}\right\rangle, r \in\left[0: m / 2^{s}-1\right], s \in[0: \log m]$, a canonical substring. In particular, both the whole string $a$, and every one of its individual characters, are canonical substrings. Every substring of $a$ can be decomposed into a concatenation of $O(\log m)$ canonical substrings.

In the following, by processing a substring $a^{\prime}$ of $a$, we mean computing the string-substring seaweed submatrix $P_{a^{\prime}, b}^{s u b}$.

First phase. We process all canonical substrings of $a$ by recursion on $m$, with one-character substrings at the base of the recursion.

Recursion base. For $m=1$, matrix $P_{a, b}^{s-s u b}$ is computed by a linear sweep of string $b$.

Recursive step. For $m>1$, we have $a=a^{\prime} a^{\prime \prime}$, where substrings $a^{\prime}, a^{\prime \prime}$ are canonical. We call the first phase recursively on each of $a^{\prime}, a^{\prime \prime}$ against $b$, obtaining matrices $P_{a^{\prime}, b}^{s-s u b}, P_{a^{\prime \prime}, b}^{s-s u b}$. Then, we obtain the matrix $P_{a, b}^{s-s u b}$ by Theorem 10.

End of recursive step.

Second phase. We represent each prescribed substring $a\langle i, j\rangle$ by a prescribed point $\left(i+\frac{1}{2}, j-\frac{1}{2}\right) \in\langle 0: m \mid 0: m\rangle$. We then partition the range $[0: m \mid 0$ : $m$ ] recursively into regular half-sized square blocks, as long as these blocks contain any prescribed points. Given indices $i_{0}, j_{0} \in[0: m]$ and block size $h$, a block is defined by the range $\left\langle i_{0}-h: i_{0} \mid j_{0}: j_{0}+h\right\rangle$. The blocks with no prescribed points, as well as $1 \times 1$ blocks containing a prescribed point, are the base of the recursion.

Throughout the recursion, we maintain the following invariant: either $j_{0}-i_{0} \leq 0$, or the substring $a\left\langle i_{0}: j_{0}\right\rangle$ has been processed, and we have the matrix $P_{a\left\langle i_{0}: j_{0}\right\rangle, b}^{s-s u b}$.

At the beginning of a recursive call, we establish whether the current block contains any prescribed points. This check is easy to perform in constant time: the current block contains a positive number of prescribed points, iff $j_{0}-i_{0}<w<j_{0}-i_{0}+2 h$. 
Recursion base. If the number of prescribed points in the current block is zero, we do nothing and return from the current recursive call.

The current block now contains at least one prescribed point. If $h=1$, then it contains exactly one prescribed point $\left(i_{0}-\frac{1}{2}, j_{0}+\frac{1}{2}\right)$, which corresponds to substring $a\left\langle i_{0}-1: j_{0}+1\right\rangle$. By the invariant, we already have the matrix $P_{a\left\langle i_{0}: j_{0}\right\rangle, b}^{s-s u b}$. Since the one-character substrings $a\left(i_{0}-\frac{1}{2}\right), a\left(j_{0}+\frac{1}{2}\right)$ are canonical, we also already have the matrices $P_{a\left(i_{0}-\frac{1}{2}\right), b}^{s-s u b}, P_{a\left(j_{0}+\frac{1}{2}\right), b}^{s-s u b}$. We can now obtain the matrix

$$
P_{a\left\langle i_{0}-1: j_{0}+1\right\rangle, b}^{s-s u b}=P_{a\left(i_{0}-\frac{1}{2}\right) a\left\langle i_{0}: j_{0}\right\rangle a\left(j_{0}+\frac{1}{2}\right), b}^{s-s u b}
$$

by two applications of Theorem 10 , with $m^{\prime}=1$ and $m^{\prime \prime}=1$ respectively.

Recursive step. We partition the current block into four $\frac{h}{2} \times \frac{h}{2}$ regular subblocks. By the invariant, we already have the matrix $P_{a\left\langle i_{0}: j_{0}\right\rangle, b}^{s-s u b}$. Since the substrings $a\left\langle i_{0}-\frac{h}{2}: i_{0}\right\rangle, a\left\langle j_{0}: j_{0}+\frac{h}{2}\right\rangle$ are canonical, we also already have the matrices $P_{a\left\langle i_{0}-\frac{h}{2}: i_{0}\right\rangle, b}^{s-s u b}, P_{a\left\langle j_{0}: j_{0}+\frac{h}{2}\right\rangle, b}^{s-s u b}$. We can now obtain the matrices

$$
\begin{array}{ll}
P_{a\left\langle i_{0}-\frac{h}{2}: j_{0}\right\rangle, b}^{s-s u b} & =P_{a\left\langle i_{0}-\frac{h}{2}: i_{0}\right\rangle a\left\langle i_{0}: j_{0}\right\rangle, b}^{s-s u b} \\
P_{a\left\langle i_{0}: j_{0}+\frac{h}{2}\right\rangle, b}^{s-s u b} & =P_{a\left\langle i_{0}: j_{0}\right\rangle a\left\langle j_{0}: j_{0}+\frac{h}{2}\right\rangle, b}^{s-s u b} \\
P_{a\left\langle i_{0}-\frac{h}{2}: j_{0}+\frac{h}{2}\right\rangle, b}^{s-s u b} & =P_{a\left\langle i_{0}-\frac{h}{2}: j_{0}\right\rangle a\left\langle j_{0}: j_{0}+\frac{h}{2}\right\rangle, b}^{s-s u b}=P_{a\left\langle i_{0}-\frac{h}{2}: i_{0}\right\rangle a\left\langle i_{0}: j_{0}+\frac{h}{2}\right\rangle, b}^{s-s u b}
\end{array}
$$

by three applications of Theorem 10, each having either $m^{\prime}=\frac{h}{2}$, or $m^{\prime \prime}=\frac{h}{2}$.

We have established the invariant for each of the four subblocks. We now make recursive calls on the subblocks.

End of recursive step.

\section{Cost analysis.}

First phase. The running time is dominated by the bottom level of the recursion tree, and is therefore $m / 2 \cdot O(n)=O(n m)$.

Second phase. The recursion tree is an unbalanced tree of degree 4 and of height $\log m$. Consider a level corresponding to block size $h$. Since all the prescribed points lie on a single diagonal, there can be at most $2 \mathrm{~m} / \mathrm{h}$ nodes at the current level, each corresponding to a block containing a positive number of prescribed points. The amount of work in every such node is $O(n \log h)$, hence the amount of work per level is $O(2 m / h \cdot n \log h)=O(n m / h \cdot \log h)$. The running time for the whole recursion tree is dominated by the bottom level $(h=1)$, and is therefore $O(n m)$.

Total. The running time for both the first and the second phase, and therefore the overall running time, is $O(m n)$.

Note that the asymptotic running time of Algorithm 10 is independent of the window length $w$. 


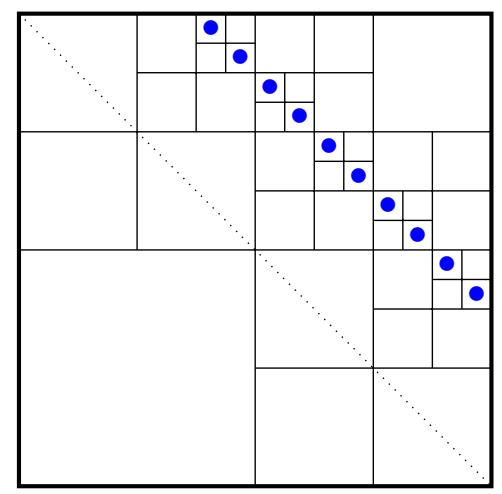

Figure 9.1: An execution of Algorithm 10 (window-substring LCS)

Example 24 Figure 9.1 shows an execution of the second stage of Algorithm 10 on a string of length 16 with window size 7 . The prescribed points are shown by blue bullets, and the resulting block partitioning by black lines.

\subsection{Quasi-local LCS}

We now consider an arbitrary set of prescribed substrings in string $a$, and denote their number by $k, m \leq k \leq m^{2}$.

Definition 27 Given strings $a, b$, the quasi-local LCS problem asks for the LCS score of every prescribed substring in $a$ against every substring in $b$. $\square$

The quasi-local LCS problem includes as special cases semi-local, windowwindow, window-substring and fully-local LCS problems, as well as lengthconstrained local alignment considered by Arslan and Ĕ̆ecioğlu [12]. The solution of the quasi-local LCS problem can be represented in space $O(k n \log n)$ by the data structure of Theorem 1, built on the string-substring seaweed submatrix for each prescribed substring of $a$ against $b$. An individual quasilocal LCS score query can be performed on this data structure in time $O\left(\log ^{2} n\right)$.

A naive algorithm for the quasi-local LCS problem runs in time $O\left(m n^{3} k\right)$. This running time can be improved by applying Algorithm 1 (the seaweed algorithm) independently to each prescribed substring $a$ against whole string $b$. The resulting algorithm runs in time $O(m n k)$. If all the prescribed substrings are sufficiently long, the running time can be improved slightly by using Algorithm 2 (the micro-block seaweed algorithm).

We now give an algorithm for the quasi-local LCS problem that provides a further improvement on the above approach. 


\section{Algorithm 11 (Quasi-local LCS)}

Input: strings $a, b$ of length $m, n$, respectively; a set of $k$ endpoint index pairs for the prescribed substrings in $a$.

Output: nonzeros of the string-substring seaweed matrix for every prescribed substring of string $a$ against full string $b$.

Description. The algorithm is based on principles similar to the ones of Algorithm 10.

First phase. As in Algorithm 10.

Second phase. Similarly to Algorithm 10, we represent each prescribed substring by a prescribed point. In order to establish efficiently the number of prescribed points located in a given block, we build a range tree [15] on the set of prescribed points, allowing efficient orthogonal range counting queries.

We then proceed by recursion as in Algorithm 10. At the beginning of a recursive call, we query from the range tree the total number of prescribed points in the current block. Otherwise, the second phase is the same as in Algorithm 10.

\section{Cost analysis.}

First phase. As in Algorithm 10.

Second phase. The recursion tree is an unbalanced tree of degree 4 and of height $\log m$. Let us call a leaf of the recursion tree useful, if it corresponds to a $1 \times 1$ block containing a prescribed point, and useless if it corresponds to an empty block. Overall, the tree has $k$ useful leaves. Every internal (i.e. non-leaf) node in the tree must have a useful leaf as a descendant, therefore there are at most $k$ internal nodes per level, hence $O(k \log m)$ nodes overall. The amount of work per node in $O(n \log m)$, therefore the running time of the whole recursion tree is $O(k \log m \cdot n \log m)=O\left(n k \log ^{2} m\right)$.

For values of $k$ close to the fully-local case $k=\left(\begin{array}{c}m \\ 2\end{array}\right)=O\left(m^{2}\right)$, a sharper analysis is possible. In this case, the running time of the whole recursion tree is $O\left(n m^{2}\right)$.

Total. The overall running time is dominated by the second phase, and is therefore $O\left(n k \log ^{2} m\right)$. For values of $k$ close to $\left(\begin{array}{c}m \\ 2\end{array}\right)$, the running time is $O\left(n m^{2}\right)$.

Note that in the fully-local case, the same asymptotic time can be obtained by running $m$ independent instances of the seaweed algorithm (Algorithm 1), each instance computing the implicit highest-score matrices incrementally for $O(n)$ different substrings of $a$.

Example 25 Figure 9.2 shows an execution of the second stage of Algorithm 11, using the same conventions as Figure 9.1. 


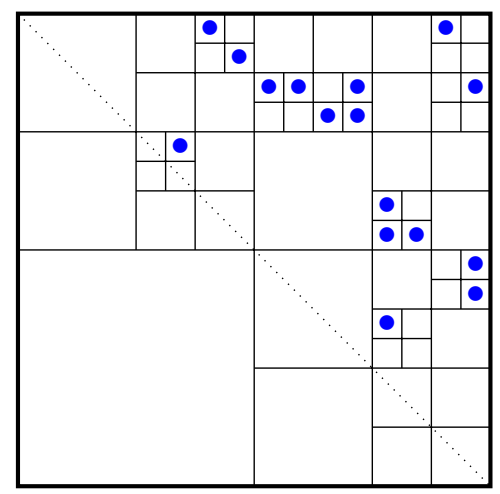

Figure 9.2: An execution of Algorithm 11 (quasi-local LCS)

\subsection{Sparse spliced alignment}

Assembling a gene from candidate exons is an important problem in computational biology. Several alternative approaches to this problem have been developed over time. One of the most successful approaches is spliced alignment by Gelfand et al. [48] (see also [54]), which scores different candidate exon chains within a DNA sequence by comparing them to a known related gene sequence. In this method, the two sequences are modelled respectively by strings $a, b$ of lengths $m, n$; we assume that $m=\Theta(n)$. A subset of substrings in string $a$ are marked as candidate exons. The comparison between sequences is made by string alignment. The algorithm for spliced alignment given in [48] runs in time $O\left(n^{3}\right)$.

In general, the number of candidate exons $k$ may be as high as $O\left(n^{2}\right)$. The method of sparse spliced alignment makes a realistic assumption that, prior to the assembly, the set of candidate exons undergoes some filtering, after which only a small fraction of candidate exons remains. Kent et al. [66] give an algorithm for sparse spliced alignment that, in the special case $k=O(n)$, runs in time $O\left(n^{2.5}\right)$. For higher values of $k$, the algorithm provides a smooth transition in running time to the dense case $k=O\left(n^{2}\right)$, where its running time $O\left(n^{3}\right)$ is asymptotically equal to the algorithm of [48].

We now consider the problem of sparse spliced alignment. We keep the notation and terminology of the previous sections; in particular, candidate exons are represented by prescribed substrings of string $a$. We say that substring $a\left\langle i^{\prime}: j^{\prime}\right\rangle$ precedes substring $a\left\langle i^{\prime \prime}: j^{\prime \prime}\right\rangle$, if $j^{\prime}<i^{\prime \prime}$. A precedence chain of substrings is a chain in the partial order of substring precedence. We identify every precedence chain with the string obtained by concatenating all its component substrings in the order of precedence.

Our sparse spliced alignment algorithm is based on the efficient method of quasi-local string comparison developed in Section 9.2. This improves the 
running time of the bottleneck procedure from [66]. The algorithm also uses a generalisation of the standard network alignment method, equivalent to the one used by [66]. For simplicity, we describe our algorithm under LCS score; using the technique of Section 3.4, the algorithm can be generalised to an arbitrary rational-weighted score.

\section{Algorithm 12 (Sparse spliced alignment)}

Input: strings $a, b$ of length $m, n$, respectively, where $m=\Theta(n)$; a set of $k$ endpoint index pairs for the prescribed substrings in $a$.

Output: the chain of prescribed substrings in $a$, giving the highest LCS score against string $b$.

Description. The algorithm runs in two phases.

First phase. By running Algorithm 11, we compute the string-substring seaweed matrix for every prescribed substring of $a$ against $b$.

Second phase. We represent the problem by a dag (directed acyclic graph) on the set of nodes $u_{i}$, where $i \in[0: m]$. For each prescribed substring $a\langle i: j\rangle$, the dag contains the edge $u_{i-1} \rightarrow u_{j}$. Overall, the dag contains $k=O(n)$ edges.

The problem can now be solved by dynamic programming on the dag as follows. Let $s(i, j)$ denote the highest LCS score across all precedence chains of prescribed substrings in prefix string $a 1 i$, taken against prefix string $b 1 j$. With each node $u_{i}$, we associate the integer vector $s_{i}=s(i, *)$. The nodes are processed in increasing order of their indices. For the node $u_{0}$, vector $s_{0}$ is initialised by all zeros. For a node $u_{j}$, we consider every edge $u_{i-1} \rightarrow u_{j}$, and compute the implicit vector-matrix distance product $s_{i-1} \unrhd H_{a\langle i: j\rangle, b}$ by the algorithm of Theorem 6 . Vector $s_{j}$ is now obtained by taking the elementwise maximum between vector $s_{j-1}$ and all the above vector-matrix distance products.

The solution score is given by the value $s_{m}(n)=s(m, n)$. The solution precedence chain of prescribed substrings can now be obtained by tracing the dynamic programming sequence backwards from node $u_{m}$ to node $u_{0}$.

\section{Cost analysis.}

First phase. Algorithm 11 runs in time $O\left(n k \log ^{2} n\right)$.

Second phase. For each of the $k$ edges in the dag, the algorithm of Theorem 6 runs in time $O(n \log n)$. Therefore, the total running time of this phase is $k \cdot O(n \log n)=O(n k \log n)$.

Total. The overall running time of the algorithm is dominated by the first phase, and is therefore $O\left(n k \log ^{2} n\right)$.

Similarly to Algorithm 11, a sharper analysis for $k \approx\left(\begin{array}{c}m \\ 2\end{array}\right)$ leads to a smooth transition to the running time $O\left(n^{3}\right)$ in the dense case $k=\left(\begin{array}{c}m \\ 2\end{array}\right)$, 
which is asymptotically equal to the running time of the dense spliced algorithm of [48]. 


\section{Chapter 10}

\section{Conclusions}

We have surveyed a number of existing and new algorithmic techniques and applications related to semi-local string comparison. Our approach unifies a substantial number of previously unrelated problems and techniques, and in many cases allows us to match or improve existing algorithms. It is likely that further development of this approach will give it even more scope and power.

A number of questions related to the semi-local string comparison framework remain open. In particular, it is not yet clear whether the framework can be extended to arbitrary real costs, or to sequence alignment with nonlinear gap penalties.

In summary, semi-local string comparison turns out to be a useful algorithmic plug-in, which unifies, and often improves on, a number of previous approaches to various substring- and subsequence-related problems. 


\section{Chapter 11}

\section{Acknowledgement}

This work was conceived in a discussion with Gad Landau in Haifa. The imaginative term "seaweeds" was coined by Yu. V. Matiyasevich during an author's presentation in St. Petersburg. The author thanks Philip Bille, Peter Krusche, Gad Landau, Victor Levandovsky, Nikolai Vavilov, and Michal Ziv-Ukelson for fruitful discussions, and the anonymous referees of his previous papers, whose comments helped to improve this work. 


\section{Bibliography}

[1] A. Aggarwal, M. M. Klawe, S. Moran, P. Shor, and R. Wilber. Geometric applications of a matrix-searching algorithm. Algorithmica, 2(1):195-208, 1987.

[2] A. V. Aho, J. E. Hopcroft, and J. D. Ullman. The Design and Analysis of Computer Algorithms. Addison-Wesley, 1976.

[3] A. V. Aho, D. S.Hirschberg, and J. D. Ullman. Bounds on the complexity of the longest common subsequence problem. Journal of the ACM, 23:1-12, 1976.

[4] M. H. Albert, R. E. L. Aldred, M. D. Atkinson, H. P. van Ditmarsch, B. D. Handley, C. C. Handley, and J. Opatrny. Longest subsequences in permutations. Australasian Journal of Combinatorics, 28:225-238, 2003.

[5] M. H. Albert, M. D. Atkinson, D. Nussbaum, J.-R. Sack, and N. Santoro. On the longest increasing subsequence of a circular list. Information Processing Letters, 101:55-59, 2007.

[6] M. H. Albert, A. Golynski, A. M. Hamel, A. López-Ortiz, S. S. Rao, and M. A. Safari. Longest increasing subsequences in sliding windows. Theoretical Computer Science, 321:405-414, 2004.

[7] C. E. R. Alves, E. N. Cáceres, and S. W. Song. An all-substrings common subsequence algorithm. Discrete Applied Mathematics, 156(7):1025-1035, 2008.

[8] A. Amir, G. Benson, and M. Farach. Let sleeping files lie: Pattern matching in Z-compressed files. Journal of Computer and System Sciences, 52(2):299-307, 1996.

[9] A. Apostolico, M. J. Atallah, and S. E. Hambrusch. New clique and independent set algorithms for circle graphs. Discrete Applied Mathematics, 36:1-24, 1992.

[10] A. Apostolico and C. Guerra. The longest common subsequence problem revisited. Algorithmica, 2(1):315-336, 1987. 
[11] V. L. Arlazarov, E. A. Dinic, M. A. Kronrod, and I. A. Faradzev. On economical construction of the transitive closure of an oriented graph. Soviet Mathematical Doklady, 11:1209-1210, 1970.

[12] A. N. Arslan and Ö. Eğecioğlu. Approximation algorithms for local alignment with length constraints. International Journal of Foundations of Computer Science, 13(5):751-767, 2002.

[13] A. N. Arslan, Ö. Eğecioğlu, and P. A. Pevzner. A new approach to sequence comparison: Normalized sequence alignment. Bioinformatics, 17(4):327-337, 2001.

[14] G. Benson. Tandem cyclic alignment. Discrete Applied Mathematics, 146(2):124-133, 2005.

[15] J. L. Bentley. Multidimensional divide-and-conquer. Communications of the ACM, 23(4):214-229, 1980.

[16] L. Bergroth, H. Hakonen, and T. Raita. A survey of longest common subsequence algorithms. In Proceedings of the 7th SPIRE, pages 3948, 2000.

[17] S. Bespamyatnikh and M. Segal. Enumerating longest increasing subsequences and patience sorting. Information Processing Letters, 76:711, 2000.

[18] P. Bille and M. Farach-Colton. Fast and compact regular expression matching. Theoretical Computer Science, 409(3):486-496, 2008.

[19] P. Bille and I. L. Gørtz. Matching subsequences in trees. Journal of Discrete Algorithms, 7(3):306-314, 2009.

[20] N. Bourbaki. Groupes et algèbres de Lie. Chapitres 4,5 et 6 . Hermann, 1968.

[21] H. Bunke and U. Bühler. Applications of approximate string matching to 2D shape recognition. Pattern Recognition, 26(12):1797-1812, 1993.

[22] R. E. Burkard, B. Klinz, and R. Rudolf. Perspectives of Monge properties in optimization. Discrete Applied Mathematics, 70(2):95-161, 1996.

[23] S. Cabello and E. W. Chambers. Multiple-source shortest paths in a genus $g$ graph. In Proceedings of the 18th ACM-SIAM SODA, pages 89-97, 2007.

[24] P. Cégielski, I. Guessarian, Y. Lifshits, and Y. Matiyasevich. Window subsequence problems for compressed texts. In Proceedings of CSR, 
volume 3967 of Lecture Notes in Computer Science, pages 127-136, 2006 .

[25] P. Cégielski, I. Guessarian, and Y. Matiyasevich. Multiple serial episodes matching. Information Processing Letters, 98(6):211-218, 2006.

[26] T. M. Chan. More algorithms for all-pairs shortest paths in weighted graphs. In Proceedings of the 39th ACM STOC, pages 590-598, 2007.

[27] Maw-Shang Chang and Fu-Hsing Wang. Efficient algorithms for the maximum weight clique and maximum weight independent set problems on permutation graphs. Information Processing Letters, 43(6):293-295, 1992.

[28] Kun-Mao Chao and Louxin Zhang. Sequence Comparison: Theory and Methods, volume 7 of Computational Biology Series. Springer, 2009.

[29] E. Chen, L. Yang, and H. Yuan. Longest increasing subsequences in windows based on canonical antichain partition. Theoretical Computer Science, 378(3):223-236, 2007.

[30] CLC bio. CLC Protein Workbench: User Manual, 2006.

[31] R. Cole and R. Hariharan. Approximate string matching: A simpler faster algorithm. SIAM Journal on Computing, 31(6):1761-1782, 2002.

[32] G. Cormode and S. Muthukrishnan. The string edit distance matching problem with moves. ACM Transactions on Algorithms, 3(1), 2007.

[33] M. Crochemore, G. M. Landau, B. Schieber, and M. Ziv-Ukelson. Reuse dynamic programming for sequence alignment: An algorithmic toolkit. In String Algorithmics, volume 2 of Texts in Algorithmics. King's College Publications, 2004.

[34] M. Crochemore, G. M. Landau, and M. Ziv-Ukelson. A subquadratic sequence alignment algorithm for unrestricted score matrices. SIAM Journal on Computing, 32(6):1654-1673, 2003.

[35] M. Crochemore and W. Rytter. Text Algorithms. Oxford University Press, 1994.

[36] G. Das, R. Fleischer, L. Gasieniec, D. Gunopulos, and J. Kärkkäinen. Episode matching. In Proceedings of CPM, volume 1264 of Lecture Notes in Computer Science, pages 12-27, 1997.

[37] E. Davydov and S. Batzoglou. A computational model for RNA multiple structural alignment. Theoretical Computer Science, 368(3):205216, 2006. 
[38] M. Demange, T. Ekim, and D. de Werra. A tutorial on the use of graph coloring for some problems in robotics. European Journal of Operational Research, 192(1):41-55, 2009.

[39] S. Deorowicz. An algorithm for solving the longest increasing circular subsequence problem. Information Processing Letters, 109(12):630$634,2009$.

[40] E. W. Dijkstra. Some beautiful arguments using mathematical induction. Acta Informatica, 13(1):1-8, 1980.

[41] A. Dress, J. H. Koolena, and V. Moulton. On line arrangements in the hyperbolic plane. European Journal of Combinatorics, 23(5):549-557, 2002 .

[42] P. Erdös and G. Szekeres. A combinatorial problem in geometry. Compositio Mathematica, 2:463-470, 1935.

[43] S. Even and A. Itai. Queues, stacks and graphs. In Theory of Machines and Computations, pages 71-86. Academic Press, 1971.

[44] G. Fertin, D. Hermelin, R. Rizzi, and S. Vialette. Common structured patterns in linear graphs: Approximation and combinatorics. In Proceedings of CPM, volume 4580 of Lecture Notes in Computer Science, pages 241-252, 2007.

[45] V. A. Fischetti, G. M. Landau, P. H. Sellers, and J. P. Schmidt. Identifying periodic occurrences of a template with applications to protein structure. Information Processing Letters, 45(1):11-18, 1993.

[46] M. L. Fredman. On computing the length of longest increasing subsequences. Discrete Mathematics, 11:29-35, 1975.

[47] F. Gavril. Algorithms for a maximum clique and a maximum independent set of a circle graph. Networks, 3:261-273, 1973.

[48] M. S. Gelfand, A. A. Mironov, and P. A. Pevzner. Gene recognition via spliced sequence alignment. Proceedings of the National Academy of Sciences of the USA, 93(17):9061-9066, 1996.

[49] R. Giancarlo. Dynamic programming: Special cases. In A. Apostolico and Z. Galil, editors, Pattern Matching Algorithms, chapter 7, pages 201-236. Oxford University Press, 1997.

[50] A. J. Gibbs and G. A. McIntyre. The diagram, a method for comparing sequences. European Journal of Biochemistry, 16(1):1-11, 1970. 
[51] M. C. Golumbic. Algorithmic Graph Theory and Perfect Graphs. Number 57 in Annals of Discrete Mathematics. Elsevier, second edition, 2004 .

[52] M. Gondran and M. Minoux. Graphs, Dioids and Semirings. Springer, 2008.

[53] O. Gotoh. An improved algorithm for matching biological sequences. Journal of Molecular Biology, 162(3):705-708, 1982.

[54] D. Gusfield. Algorithms on Strings, Trees, and Sequences: Computer Science and Computational Biology. Cambridge University Press, 1997.

[55] D. Hermelin, G. M. Landau, S. Landau, and O. Weimann. A unified algorithm for accelerating edit-distance computation via textcompression. In Proceedings of the 26th STACS, pages 529-540, 2009.

[56] D. S. Hirschberg. A linear space algorithm for computing maximal common subsequences. Communications of the ACM, 18(6):341-343, 1975.

[57] W.-L. Hsu. Maximum weight clique algorithms for circular-arc graphs and circle graphs. SIAM Journal on Computing, 14(1):224-231, 1985.

[58] Yue Huang and Ling Zhang. Rapid and sensitive dot-matrix methods for genome analysis. Bioinformatics, 20(4):460-466, 2004.

[59] J. W. Hunt and T. G. Szymanski. A fast algorithm for computing longest common subsequences. Communications of the ACM, 20(5):350-353, 1977.

[60] Y. Ishida, S. Inenaga, A. Shinohara, and M. Takeda. Fully incremental LCS computation. In Proceedings of FCT, volume 3623 of Lecture Notes in Computer Science, pages 563-574, 2005.

[61] B. N. Jackson and S. Aluru. Pairwise sequence alignment. In Handbook of Computational Molecular Biology, Chapman and Hall/CRC Computer and Information Science Series, chapter 1, pages 1-1 - 1-31. Chapman and Hall/CRC, 2006.

[62] J. JáJá, C. Mortensen, and Q. Shi. Space-efficient and fast algorithms for multidimensional dominance reporting and counting. In Proceedings of the 15th ISAAC, volume 3341 of Lecture Notes in Computer Science, pages 558-568, 2004.

[63] N. C. Jones and P. A. Pevzner. An introduction to bioinformatics algorithms. Computational Molecular Biology. The MIT Press, 2004. 
[64] A. Karzanov. Combinatorial methods to solve cut-determined multiflow problems. In Combinatorial Methods for Flow Problems, volume 3, pages 6-69. VNIISI, 1979. In Russian.

[65] C. Kassel and V. Turaev. Braid Groups, volume 247 of Graduate Texts in Mathematics. Springer, 2008.

[66] C. Kent, G. M. Landau, and M. Ziv-Ukelson. On the complexity of sparse exon assembly. Journal of Computational Biology, 13(5):10131027, 2006.

[67] S.-R. Kim and K. Park. A dynamic edit distance table. Journal of Discrete Algorithms, 2(2):303-312, 2004.

[68] P. N. Klein. Multiple-source shortest paths in planar graphs. In Proceedings of the 16th ACM-SIAM SODA, pages 146-155, 2005.

[69] D. E. Knuth. Permutations, matrices, and generalized Young tableaux. Pacific Journal of Mathematics, 34(3):709-727, 1970.

[70] A. Kosowski. An efficient algorithm for the longest tandem scattered subsequence problem. In Proceedings of the 11th SPIRE, volume 3246 of Lecture Notes in Computer Science, pages 93-100, 2004.

[71] J. Krumsiek, R. Arnold, and T. Rattei. Gepard: A rapid and sensitive tool for creating dotplots on genome scale. Bioinformatics, 23(8):1026$1028,2007$.

[72] P. Krusche and A. Tiskin. String comparison by transposition networks. In London Algorithmics 2008: Theory and Practice, volume 11 of Texts in Algorithmics. College Publications, 2009.

[73] G. Landau. Can DIST tables be merged in linear time? An open problem. In Proceedings of the Prague Stringology Conference, page 1. Czech Technical University in Prague, 2006.

[74] G. M. Landau, E. Myers, and M. Ziv-Ukelson. Two algorithms for LCS consecutive suffix alignment. Journal of Computer and System Sciences, 73(7):1095-1117, 2007.

[75] G. M. Landau, E. W. Myers, and J. P. Schmidt. Incremental string comparison. SIAM Journal on Computing, 27(2):557-582, 1998.

[76] G. M. Landau and U. Vishkin. Fast parallel and serial approximate string matching. Journal of Algorithms, 10(2):157-169, 1989.

[77] G. M. Landau and M. Ziv-Ukelson. On the common substring alignment problem. Journal of Algorithms, 41(2):338-359, 2001. 
[78] V. Levenshtein. Binary codes capable of correcting spurious insertions and deletions of ones. Problems of Information Transmission, 1:8-17, 1965 .

[79] Y. Lifshits. Processing compressed texts: A tractability border. In Proceedings of CPM, volume 4580 of Lecture Notes in Computer Science, pages 228-240, 2007.

[80] Y. Lifshits and M. Lohrey. Querying and embedding compressed texts. In Proceedings of MFCS, volume 4162 of Lecture Notes in Computer Science, pages 681-692, 2006.

[81] M. Maes. On a cyclic string-to-string correction problem. Information Processing Letters, 35:73-78, 1990.

[82] J. V. Maizel and R. P. Lenk. Enhanced graphic matrix analysis of nucleic acid and protein sequences. Proceedings of the National Academy of Sciences of the USA, 78(12):7665-7669, 1981.

[83] A. Marzal and S. Barrachina. Speeding up the computation of the edit distance for cyclic strings. In Proceedings of the 15th ICPR, part 2, pages 891-894, 2000.

[84] W. J. Masek and M. S. Paterson. A faster algorithm computing string edit distances. Journal of Computer and System Sciences, 20:18-31, 1980.

[85] S. Masuda, K. Nakajima, T. Kashiwabara, and T. Fujisawa. Efficient algorithms for finding maximum cliques of an overlap graph. Networks, 20:157-171, 1990.

[86] C. Mueller, M. M. Dalkilic, and A. Lumsdaine. High-performance direct pairwise comparison of large genomic sequences. IEEE Transactions on Parallel and Distributed Systems, 17(8):764-772, 2006.

[87] E. W. Myers and W. Miller. Approximate matching of regular expressions. Bulletin of Mathematical Biology, 51(1):5-37, 1989.

[88] G. Myers. Approximately matching context-free languages. Information Processing Letters, 54:85-92, 1995.

[89] G. Navarro. A guided tour to approximate string matching. ACM Computing Surveys, 33(1):31-88, 2001.

[90] S. B. Needleman and C. D. Wunsch. A general method applicable to the search for similarities in the amino acid sequence of two proteins. Journal of Molecular Biology, 48(3):443-453, 1970. 
[91] S. Ott, S. Gunawardana, M. Downey, and G. Koentges. Loss-free identification of alignment-conserved CRMs. In preparation.

[92] M. S. Paterson and V. Dančík. Longest common subsequences. In Proceedings of MFCS, volume 841 of Lecture Notes In Computer Science, pages $127-142,1994$.

[93] F. P. Preparata and M. I. Shamos. Computational Geometry: An Introduction. Texts and Monographs in Computer Science. Springer, 1985.

[94] C. Putonti, Yi Luo, C. Katili, S. Chumakov, G. E. Fox, D. Graur, and Y. Fofanov. A computational tool for the genomic identification of regions of unusual compositional properties and its utilization in the detection of horizontally transferred sequences. Molecular Biology and Evolution, 23(10):1863-1868, 2006.

[95] P. Rice, I. Longden, and A. Bleasby. EMBOSS: The European molecular biology open software suite. Trends in Genetics, 16(6):276-277, 2000 .

[96] R. W. Richardson and T. A. Springer. The Bruhat order on symmetric varieties. Geometriae Dedicata, 35(1-3):389-436, 1990.

[97] G. de B. Robinson. On the representations of the symmetric group. American Journal of Mathematics, 60(3):745-760, 1938.

[98] G. Rote. Path problems in graphs. Computing Supplementum, 7:155$189,1990$.

[99] D. Rotem and J. Urrutia. Finding maximum cliques in circle graphs. Networks, 11:269-278, 1981.

[100] W. Rytter. Algorithms on compressed strings and arrays. In Proceeedings of SOFSEM, volume 1725 of Lecture notes in Computer Science, pages 48-65, 1999.

[101] J. P. Schmidt. All highest scoring paths in weighted grid graphs and their application to finding all approximate repeats in strings. SIAM Journal on Computing, 27(4):972-992, 1998.

[102] P. H. Sellers. The theory and computation of evolutionary distances: Pattern recognition. Journal of Algorithms, 1(4):359-373, 1980.

[103] T. F. Smith and M. S. Waterman. Identification of common molecular subsequences. Journal of Molecular Biology, 147(1):195-197, 1981. 
[104] E. L. L. Sonnhammer and R. Durbin. A dot-matrix program with dynamic threshold control suited for genomic DNA and protein sequence analysis. Gene, 167(1-2):GC1-GC10, 1995.

[105] A. Tiskin. All semi-local longest common subsequences in subquadratic time. In Proceedings of CSR, volume 3967 of Lecture Notes in Computer Science, pages 352-363, 2006.

[106] A. Tiskin. Longest common subsequences in permutations and maximum cliques in circle graphs. In Proceedings of CPM, volume 4009 of Lecture Notes in Computer Science, pages 271-282, 2006.

[107] A. Tiskin. Semi-local longest common subsequences in subquadratic time. Journal of Discrete Algorithms, 6(4):570-581, 2008.

[108] A. Tiskin. Semi-local string comparison: Algorithmic techniques and applications. Mathematics in Computer Science, 1(4):571-603, 2008.

[109] A. Tiskin. Faster subsequence recognition in compressed strings. Journal of Mathematical Sciences, 158(5):759-769, 2009.

[110] A. Tiskin. Periodic string comparison. In Proceedings of CPM, volume 5577 of Lecture Notes in Computer Science, pages 193-206, 2009.

[111] S. Tsaranov. Representation and classification of Coxeter monoids. European Journal of Combinatorics, 11(2):189-204, 1990.

[112] R. A. Wagner and M. J. Fischer. The string-to-string correction problem. Journal of the ACM, 21(1):168-173, 1974.

[113] B. W. Watson and G. Zwaan. A taxonomy of sublinear multiple keyword pattern matching algorithms. Science of Computer Programming, 27(2):85-118, 1996.

[114] T. A. Welch. A technique for high-performance data compression. Computer, 17(6):8-19, 1984.

[115] S. Wu, U. Manber, and G. Myers. A subquadratic algorithm for approximate limited expression matching. Algorithmica, 15:50-67, 1996.

[116] G. Ziv and A. Lempel. A universal algorithm for sequential data compression. IEEE Transactions on Information Theory, 23:337-343, 1977.

[117] G. Ziv and A. Lempel. Compression of individual sequences via variable-rate coding. IEEE Transactions on Information Theory, 24:530-536, 1978. 


\section{Index}

$(I \mid J)$ : Cartesian product, 6

$*$ : implicit range, 7

$A^{\square}$ : density matrix, 8

$D^{\Sigma}$ : distribution matrix, 8

$G_{a, b}$ : alignment dag, 30

$H_{a, b}$ : semi-local score matrix, 32

$P_{a, b}$ : semi-local seaweed matrix, 33

$I d$ : identity matrix, 9

$I d_{h}$ : offset indentity matrix, 9

$\Sigma(a)$ : character set, 62

$\langle i: j\rangle$ : odd half-integer interval, 6

$\left\langle i_{0}: i_{1} \mid j_{0}: j_{1}\right\rangle:$ interval Cartesian product, 6

$\bar{a}$ : string reverse, 62

$\ulcorner$ : implicit distance multiplication, 15

$[i: j]$ : integer interval, 6

$\left[i_{0}: i_{1} \mid j_{0}: j_{1}\right]$ : interval Cartesian product, 6

$\because$ induced subrange, 9

$i d$ : identity permutation string, 62

।, l: left/right drop, 28

$\ll, \lessgtr$ : dominance orders, 6

1, $\uparrow:$ left/right take, 28

$\odot$ : distance multiplication, 12

$\sqcup$ : matrix disjoint union, 7

$\sqsupset$ : matrix embedding, 7

$\diamond$ : wildcard character, 28

$\left.a\right|_{S}$ : filtered subsequence, 62

\$: non-alphabet character, 28

algorithm

dynamic programming, 44, 55

micro-block, 50

wraparound, 61

seaweed, 44

micro-block, 51

periodic, 58

Smith-Waterman-Gotoh, 81

alignment dag, 30

alignment score

LCS, 29

weighted, 40

normalised, 41

rational, 42

antichain, 6

chain, 6

circle graph, 67

interval model, 67

distance multiplication

matrix-matrix, 12

implicit, 15

matrix-vector, 12

implicit, 15

dominance, 6

counting, 10

dot plot

alignment-filtered, 82

Hamming-filtered, 81

edit distance, 42

episode, 42

LCS, 42

Levenshtein, 42

function

unit-monotone, 6

Hamming score, 81

interval notation, 6 
linear graph, 71

matrix

anti-Monge, 8

density, 8

distribution, 8

general

$\odot$-identity, 12

$\odot$-monoid, 12

highest-score

composition, 36

implicit, 7

batch query, 11

element query, 10

incremental query, 11

Monge, 8

$\odot$-identity, 14

$\odot$-monoid, 13

periodic, 57

period submatrix, 58

permutation, 9

identity, 9

offset indentity, 9

superdiagonal, 9

score

prefix-suffix, 34

semi-local, 32

string-substring, 34

substring-string, 34

suffix-prefix, 34

seaweed

prefix-suffix, 35

semi-local, 33

string-substring, 35

substring-string, 35

suffix-prefix, 35

simple, 8

subpermutation, 9

subunit-anti-Monge, 9

subunit-Monge, 9

totally monotone, 8

unit-anti-Monge, 9

unit-Monge, 9

๑-identity, 14 $\odot$-monoid, 13

problem

approximate pattern matching, 54 complete, 55

threshold, 55

block-incremental LCS, 47

circle graph maximum clique, 68

common-substring LCS, 48

semi-local, 48

cyclic LCS, 53

between permutations, 64

fully-incremental LCS, 47

incremental LCS, 47

linear graph maximum common pattern, 71

longest $k$-increasing subsequence, 66

longest $k$-modal subsequence, 66

longest $Y$-avoiding subsequence, 65

longest common subsequence (LCS), 29

longest increasing subsequence (LIS), 62

longest repeating subsequence, 54

periodic string-substring LCS, 57

quasi-local LCS, 85

semi-local LCS, 29

on GC-strings, 75

three-way, 30

subsequence recognition

global, 28

local, 29

local (fixed-window), 55

local (minimal-window), 54

on GC-strings, 74, 77

tandem alignment, 60

tandem LCS, 59

window-substring LCS, 82

window-window LCS, 82

seaweed, 15

braid, 15 
monoid, 16

spliced alignment, 87

sparse, 87

straight-line program (SLP), 73

statement, 73

string

grammar-compressed (GC), 73

periodic, 57

permutation, 62

identity, 62

prefix, 28

reverse, 62

subsequence, 28

substring, 28

suffix, 28

window, 81

subsequence

filtered, 62

substring

canonical, 83

overlap, 36

prescribed, 81 